Portland State University

PDXScholar

$11-18-2020$

\title{
A Posteriori Error Estimates for Maxwell's Equations Using Auxiliary Subspace Techniques
}

Ahmed El sakori

Portland State University

Follow this and additional works at: https://pdxscholar.library.pdx.edu/open_access_etds

Part of the Mathematics Commons

Let us know how access to this document benefits you.

Recommended Citation

El sakori, Ahmed, "A Posteriori Error Estimates for Maxwell's Equations Using Auxiliary Subspace Techniques" (2020). Dissertations and Theses. Paper 5599.

https://doi.org/10.15760/etd.7471

This Dissertation is brought to you for free and open access. It has been accepted for inclusion in Dissertations and Theses by an authorized administrator of PDXScholar. Please contact us if we can make this document more accessible: pdxscholar@pdx.edu. 
A Posteriori Error Estimates for Maxwell's Equations Using Auxiliary Subspace Techniques

by

Ahmed El sakori

A dissertation submitted in partial fulfillment of the requirements for the degree of

\author{
Doctor of Philosophy \\ in \\ Mathematical Sciences
}

\author{
Dissertation Committee: \\ Jeffrey Ovall, Chair \\ Jay Gopalakrishnan \\ Bin Jiang \\ Erik Sanchez
}

Portland State University

2020 
(c) 2020 Ahmed El sakori 


\begin{abstract}
The aim of our work is to construct provably efficient and reliable error estimates of discretization error for Nédélec (edge) element discretizations of Maxwell's equations on tetrahedral meshes. Our general approach for estimating the discretization error is to compute an approximate error function by solving an associated problem in an auxiliary space that is chosen so that:

- Efficiency and reliability results for the computed error estimates can be established under reasonable and verifiable assumptions.

- The linear system used to compute the approximate error function has condition number bounded independently of the discretization parameter.

In many applications, it is some functional of the solution that is primarily of interest. In such cases, it makes sense to estimate and control discretization error with respect to this functional, rather than with respect to a global norm. We will also develop auxiliary subspace techniques for this kind of goal-oriented error estimation.
\end{abstract}




\section{Dedication}

I dedicate this dissertation to..

My precious mother and dear father, who taught me the meaning of effort and perseverance, who have been constant cheerleadrs through every academic and personal endeavor in my life, who always believed in me and encouraged me to strive for my dreams.

My life partner, for whom I have the utmost respect, who has offered unwavering support and encouragement during the past six years of my doctoral journey. Who still gives me unparalleled positive energy to make a failure to become a success, and to my life joy, my sweet kids (Ziyad, Zakaria, Anas, and Lena).

All my family and friends who lived in my life with all friendliness and respect and shared the most beautiful memories with me.

Also, I would like to express my dedication to everyone who has illuminated me with his/her knowledge or guided me by offering the right answer to my puzzling, to those who shared with me the character of life and its sorrows, to those who have waited anxiously for this moment, to those who have given me all the means of comfort to reach this educational level. 


\section{Acknowledgments}

My sincere thanks and deep gratitude go to my major professor, Dr. Jeffrey Ovall. Dr. Ovall, you have been an outstanding mentor and friend to me during my work on the doctoral thesis and my graduate studies. Thank you for your kindness, gentle prodding, and unwavering confidence in my ability to get this done. Without your guidance and persistent help, this dissertation would not have been possible.

I also offer thanks to my committee members, Dr. Gopalakrishnan, Dr. Jiang, and Dr. Sanchez. Thanks to each of you for helpful discussions and feedback in evaluating my work. I especially want to thank Dr. Gopalakrishnan for his invaluable advice and wealth of knowledge on Netgen/NGSolve software that helped guide me through running my code.

To my past and present graduate students at the Fariborz Maseeh Department of Mathematics and Statistics, I offer my sincere thanks for your aid, understanding, and encouragement over these past several years and create an enjoyable environment in which to discuss and work. I have gained so much from our many meetings and discussions.

This thesis has only been possible with the financial sponsorship I received from the Ministry of Higher Education and Scientific Research in my country Libya. Thanks to all of you!

Finally, I am pleased to thank the administration, faculty, and students at the Fariborz Maseeh Department of Mathematics and Statistics to be a member of your big family. 
Table of Contents

Abstract

Dedication ii

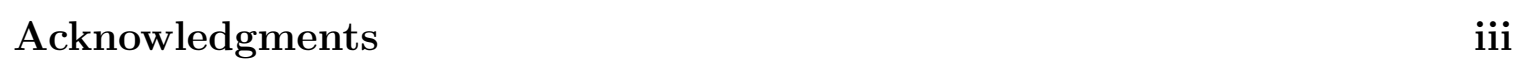

List of Tables $\quad$ vi

List of Figures vii

\begin{tabular}{lll}
\hline Chapter 1 & Introduction & 1
\end{tabular}

Chapter 2 Maxwell's Equations: Strong and weak formulations, well\begin{tabular}{|c|}
\hline posedness \\
\hline
\end{tabular}

2.1 Maxwell's equations . . . . . . . . . . . . . . . . . 5

2.2 Time-harmonic Maxwell's equations . . . . . . . . . . . . . . . . . . . 6

2.3 Strong Form (curl-curl problem) . . . . . . . . . . . . . . . . . . . . . 8

2.4 Variational Framework . . . . . . . . . . . . . . . . . . . . . . . . . . 9

2.4 .1 Notation . . . . . . . . . . . . . . . . . . 9

2.4 .2 Function Spaces . . . . . . . . . . . . . . . . . . . . . . 9

2.4.3 Traces, Boundary Conditions and Variational Formulation . . 11

2.4 .4 Abstract Well-posedness Results for Variational Problems. . . 14

$\begin{array}{lll}\text { Chapter } 3 & \text { Finite Elements for Maxwell's Equations } & 18\end{array}$

$3.1 \quad$ Triangulation and Finite Element Spaces . . . . . . . . . . . . . . . 18

3.2 Polynomial spaces and Nédélec Space . . . . . . . . . . . . . . . . . 20

$3.3 \quad$ Barycentric Coordinates on the Tetrahedron . . . . . . . . . . . . . . 23

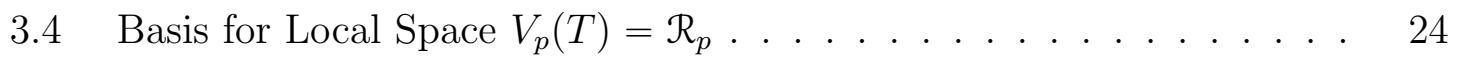

3.5 Global Spaces, Dimension and Bases . . . . . . . . . . . . . . . . . . 24

3.6 The discrete variational problem . . . . . . . . . . . . . . . . . . 26

3.7 The interpolant for $V \ldots \ldots \ldots \ldots \ldots$. . . . . . . . . . . . . 27 
$\begin{array}{lll}\text { Chapter } 4 \text { A posteriori Error Estimation } & 29\end{array}$

$4.1 \quad$ Hierarchical Based Error Estimator. . . . . . . . . . . . . . . . . . . 29

4.2 Key Error Estimation Result . . . . . . . . . . . . . . . . . . . . . 31

$4.3 \quad$ Local and Global Error Space $W \ldots \ldots \ldots \ldots$

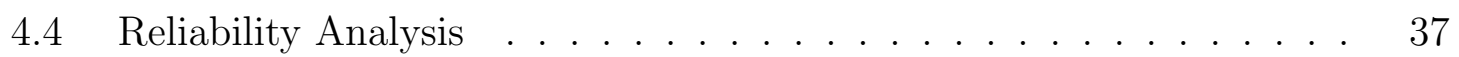

4.4 .1 Proof of Theorem| $4.3 \ldots \ldots \ldots$

4.5 Computational Cost $\ldots \ldots \ldots \ldots \ldots$

4.5 .1 Proof of Theorem $4.4 \ldots \ldots \ldots \ldots$

4.5 .2 Example . . . . . . . . . . . . . . . . . . . . . . 53

4.6 Numerical Experiments $\ldots \ldots \ldots \ldots$

4.6 .1 Example $1 \ldots \ldots \ldots \ldots \ldots \ldots$

4.6 .2 Example $2 \ldots \ldots \ldots \ldots$

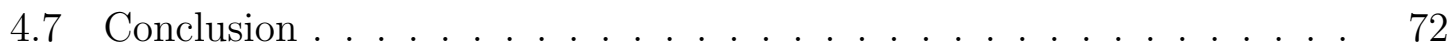

\begin{tabular}{lll}
\hline Chapter 5 & The goal-oriented error estimator & 73
\end{tabular}

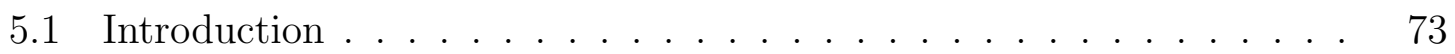

5.2 Basic Duality/Adjoint Framework . . . . . . . . . . . . . . . . 74

5.3 Adaptive Refinement Scheme for Numerical Accuracy . . . . . . . . 76

5.4 Numerical Experiments $\ldots \ldots \ldots \ldots \ldots . \ldots \ldots 77$

$5.4 .1 \quad$ Example $1 \ldots \ldots \ldots \ldots \ldots \ldots \ldots$

5.4 .2 Example 2 . . . . . . . . . . . . . . . . . . . . . . 85

5.5 Conclusion $\ldots \ldots \ldots \ldots \ldots \ldots$

\begin{tabular}{ll}
\hline References & 92
\end{tabular}

\begin{tabular}{ll}
\hline Appendix & 97
\end{tabular}

A.1 Additional Numerical Results . . . . . . . . . . . . . . . . . . . . . 97

A.2 Numerical Results in $L^{2}$ norm . . . . . . . . . . . . . . . . . . 97

A.2.1 Example 1 . . . . . . . . . . . . . . . . . . . . 97

A.2.2 $\quad$ Example 2 $\ldots \ldots \ldots \ldots 9$

A.3 Numerical Results by using Nédélec space of the second-kind . . . . . 101

A.3.1 Nédélec $H(\operatorname{curl} ; \Omega)$ element of the second-kind . . . . . . . . . 101

A.3.2 $\quad$ Example 1 . . . . . . . . . . . . . . . . . . . . 102

A.3.3 $\quad$ Example 2 . . . . . . . . . . . . . . . . . . . 107 


\section{List of Tables}

Table 3.1 The dimensions of $V_{p}(T)=\mathcal{R}_{p}$ and its gradient and gradient-free subspaces $\mathcal{R}_{p}^{G}$ and $\mathcal{R}_{p}^{F}$, together with those of the edge, face and volume subspaces $V_{p}^{E}(T), V_{p}^{F}(T), V_{p}^{T}(T)$, for small $p . \mid \ldots 22$

\begin{tabular}{|ll|l|l|} 
Table 3.2 & Bases given in $[17]$ \\
coordinates, $p \leqslant 3$
\end{tabular}

Table 4.1 Numerical errors, convergence order, and effectivity indices of the error estimator in $H$-curl norm (Example 1). . . . . . . . . . . . 59

Table 4.2 Numerical errors, convergence order, and effectivity indices of the error estimator in energy norm (Example 1). . . . . . . . . . . . 60

Table 4.3 Condition numbers of the stiffness matrices for $V$ and $W$ (Example 1). . . . . . . . . . . . . . . . 62

Table 4.4 Numerical errors, convergence order, and effectivity indices of the error estimator in $H$-curl norm (Example 2). . . . . . . . . . . . 68

Table 4.5 Numerical errors, convergence order, and effectivity indices of the error estimator in energy norm (Example 2). . . . . . . . . . . . 69

Table 4.6 Condition numbers of the stiffness matrices for $V$ and $W$ (Example 2). . . . . . . . . . . . . . . . . 71

Table 5.1 Numerical errors and effectivity indices of goal-oriented error estimator when $\beta \in\left\{-10^{2},-10,-1,1,10\right\}$ (Example 1). . . . . . . 81

Table 5.2 Numerical errors and effectivity indices of global error estimator in H-curl and energy norms when $\beta \in\left\{-10^{2},-10,-1,1,10\right\}$ (Example 1). . . . . . . . . . . . . . . . . . . 82

Table 5.3 Condition numbers of the stiffness matrices for $V$ and $W$ for $\beta \in\left\{-10^{2},-10,-1,1,10\right\}$ (Example 1). . . . . . . . . . . . 84

Table 5.4 Numerical errors and effectivity indices of goal-oriented and global estimates over $\Omega_{1}, \Omega_{2}, \Omega_{3}$, and $\Omega_{s}$ (Example 2$)$. . . . . . . . . . 88

Table 5.5 Condition numbers of the stiffness matrices for $V$ and $W$ in each choice of subdomains (Example 2). . . . . . . . . . . . . . . . . 90 


\section{List of Figures}

Figure 4.1 The initial mesh and its uniform refinements (Example 1). . . 56

Figure 4.2 Uniform Solutions (Exact Versus Approximate Solutions) (Example 1). . . . . . . . . . . . . . . . . 57

Figure 4.3 Convergence rate of the exact error in $H$-curl norm (red) and energy norm (blue) (Example 1). . . . . . . . . . . . . 61

Figure $4.4 \quad$ Effectivity indices of the error estimators in $H$-curl norm (red) and energy norm (blue) (Example 1). . . . . . . . . . . . . . . 61

Figure 4.5 Condition numbers of the stiffness matrices for $V$ (red) and $W$ (blue) (Example 1). . . . . . . . . . . . . . 63

Figure $4.6 \quad$ Coarse mesh and Adaptive Refinement Levels (Example 2). . . 65

Figure 4.7 Adaptive Solutions (Exact Versus Approximate Solutions) (Example 2). . . . . . . . . . . . . . . . . 66

Figure 4.8 Convergence rate of the exact error in $H$-curl norm (red) and energy norm (blue) (Example 2). . . . . . . . . . . . . . . . . 70

Figure 4.9 Effectivity indices of the error estimators in $H$-curl norm (red) and energy norm (blue) (Example 2). . . . . . . . . . . . . 70

Figure 4.10 Condition numbers of the stiffness matrices for $V$ (red) and $W$ (blue) (Example 2). . . . . . . . . . . . . . . . . . . . 72

Figure 5.1 The subdomain $\Omega_{s}$ of the domain $\Omega$ (Example 1). . . . . . . . 78

Figure 5.2 Adapted meshes, intermediate and final, to control $G(\mathbf{u})$ in $\Omega_{s}$ when $\beta=1$ (Example 1). . . . . . . . . . . . . . . . 79

Figure $5.3 \quad$ Approximate Dual Solutions for intermediate and final meshes for $\beta=1$ (Example 1). . . . . . . . . . . . . . . . . . 79

Figure 5.4 Error estimators using the global error (GB) and goal-oriented (GO) adaptivity strategy (Example 1). . . . . . . . . . . 83

Figure 5.5 Effectivity indices using the global error (GB) and goal-oriented (GO) adaptivity strategy (Example 1). . . . . . . . . . . . 83

Figure $5.6 \quad$ Condition numbers of the stiffness matrices for $V$ (red) and $W$ (blue) (Example 1). . . . . . . . . . . . . . . . 85

Figure 5.7 The subdomain $\Omega_{s}$ of the domain $\Omega$ (Example 2). . . . . . . 86 
Figure 5.8 The final mesh refinement over each subdomain separately and over all subdomains (Example 2). . . . . . . . . . . . . . . . . . 87

Figure 5.9 Error estimators using the global error (GB) and goal-oriented (GO) adaptivity strategy (Example 2). . . . . . . . . . . . . . . 89

Figure 5.10 Effectivity indices using the global error (GB) and goal-oriented (GO) adaptivity strategy (Example 2). . . . . . . . . . . . . . . . . . 89

Figure 5.11 Condition numbers of the stiffness matrices for $V$ (red) and $W$ (blue) (Example 2). . . . . . . . . . . . . . . . . . . 91 


\section{Chapter 1}

\section{Introduction}

Maxwell's equations describe how electric charges and electric currents create electric and magnetic fields, and how these fields propagate and influence each other. They provide a mathematical model for many recent technologies, such as power generation, electric motors, wireless communication, lenses, radar etc. Electromagnetic geophysics, more specifically the (inverse) problem of determining a realistic conductivity/resistivity model of the earth near the surface (e.g. air/earth interface or ocean/earth interface) from a series of experiments in which known sources are used to generate the EM fields, and responses are measured at an array of locations, will provide motivation for some of the choices in our development.

The use of finite element methods to approximate solutions of Maxwell's equations goes back to at least 1980 [29], when Nédélec introduced his now-famous "edge elements", which provide a natural discretization of functions in the space $H(\operatorname{curl} ; \Omega)$ in which the variatonal form of Maxwell's equations is typically posed. Having used the Nédélec (edge) element discretization to compute an approximation of the electric field (for example), it is natural to ask how well it approximates the true solution. One often quantifies this discretization error in terms of global norm. A priori error estimates describe the qualitative behavior of the error with respect to parameters governing the discretization, such as the characteristic edge length in a tetrahedral mesh. Although such qualitative estimates provide useful information, in practice one often wants more quantitative estimates of error, both to determine whether or not a discrete solution is "good enough", and to indicate how to intelligently improve the discrete solution if it is not. This is the realm of a posteriori error estimation, which 
is the focus of our research.

For standard finite element discretization of PDEs modeling diffusion processes, a posteriori error theory is a mature field, and there are many well-tested techniques on the market. In contrast, a posteriori error theory for Maxwell's equations (by Nédélec elements), is much more recent, and there are relatively few techniques in use that are both theoretically and empirically supported $[5,7,8,11,19,21,22,25,30,34,36]$. Our work will provide provably efficient and reliable error estimates of discretization error based on auxiliary subspace techniques for Nédélec (edge) element discretization of Maxwell's equations on tetrahedral meshes. Such methods have a long history in the context of standard discretization of diffusion problems, where they are often called hierarchical basis error estimators [1,3, 13, 14,37]. Such estimators are based on the computation of an approximate error function $\varepsilon \approx \mathbf{u}-\hat{\mathbf{u}}(\hat{\mathbf{u}} \in V$ is a given finite element approximation of the solution $\mathbf{u}$ ) in an auxiliary discrete space $W$ satisfying $V \cap W=\{\mathbf{0}\}$. The traditional analysis of hierarchical error estimators [2, 4], in case the bilinear form $B$ is an inner-product with induced "energy norm" $\|\mathbf{v}\|^{2}=B(\mathbf{v}, \mathbf{v})$, makes use of a strong Cauchy inequality between the spaces $V$ and $W$ and a saturation assumption to obtain upper and lower bounds on error norm $\|\mathbf{u}-\hat{\mathbf{u}}\|$, in terms of the error estimate norm $\|\varepsilon\|$. Roughly speaking, the saturation assumption asserts that $\mathbf{u}$ is (strictly) better approximated in the "enriched space" $V \oplus W$ than it is in $V$, and this perspective, though intuitive, often causes viable auxiliary spaces $W$ to be overlooked 18,20$]$.

Although the saturation assumption is expected to hold in many cases, constructing counter-examples it is not difficult for particular problems on particular meshes. Furthermore, this kind of analysis cannot be readily applied for the more general bilinear forms such as those (that are not inner-products) considered here; and, as 
suggested above, may unnecessarily limit the types of spaces $W$ in which the error might be approximated. The "saturation assumption approach" was taken in [5] to derive a hierarchical basis error estimator for Maxwell's equations, though it appears that their work has not been pursued further. In this study we will take a different approach, to obtain error bounds under weaker (and verifiable) assumptions, using different spaces than in [5]. The principle of this approach is to replace the saturation assumption by "residual oscillation", which appears explicitly in the reliability bound of the error as a computable quantity. The proof of this inequality depends primarily on the choice of the space $W$ in which the error indicator lies. We will also show that the matrix of the auxiliary problem for computing the approximate error function in $W$ is spectrally equivalent to its diagonal, and therefore much simpler to work with than the matrix for the original problem. Having established that the estimator is efficient and reliable, and computationally feasible, we will extend the approach to treat functional measures of error.

The rest of this thesis is organized as follows. In Chapter 2 we describe Maxwell's equations and introduce the strong and weak formulations. Also, at the end of this chapter, we will briefly discuss assumptions that guarantee existence and uniqueness of the weak form. The material in this chapter is standard and is presented here to make our work more self-contained. The suitable finite elements of the Sobolev space $H(\operatorname{curl} ; \Omega)$, needed for discretizing the Maxwell system, will be presented in Chapter 3. First, we will define the classical finite element spaces (FE spaces) for the weak formulation, and then we will introduce the discrete variational problem. In Chapter 4, we will introduce our key error estimation results, and will pursue the analysis of our approach theoretically and empirically. We will show that the estimate of the error is provably reliable and efficient, and the cost of computing such error 
estimates is reasonable and not much expensive. Later in this chapter, we support our arguments by numerical experiments. Lastly, in Chapter 5, we will present a new approach in a posteriori error estimation, called Goal-oriented error estimation, in which the numerical error of finite element approximations is estimated in terms of quantities of interest rather than the classical global $H$-curl or energy norm. These so-called quantities of interest are characterized by linear functionals on the space of functions to where the solution belongs. We end this chapter with numerical experiments which illustrate that the error in the goal functional is estimated very precisely and can be reduced rapidly by applying the adaptive algorithm. 


\section{Chapter 2}

\section{Maxwell's Equations: Strong and weak formulations, well-posedness}

In this chapter we will describe Maxwell's equations and introduce the strong form (curl-curl problem) of these equations, which will be our starting point for the weak formulation. Also, at the end of this chapter, we will briefly discuss assumptions that guarantee existence and uniqueness of the weak form. The material in this chapter is standard [28] and is presented here to make our work more self-contained, and to introduce some key notation and terminology.

\subsection{Maxwell's equations}

Maxwell's equations are a set of four equations that describe how electric charges and electric currents act as sources for electric and magnetic fields. Further, they describe how these fields propagate and influence each other. Maxwell's equations state that the field variables and sources are related by the following equations which apply throughout the region of space in $\mathbb{R}^{3}$ occupied by the electromagnetic field:

$$
\begin{aligned}
\frac{\partial \mathcal{B}}{\partial t}+\nabla \times \mathcal{E} & =0, \\
\nabla \cdot \mathcal{D} & =\rho, \\
\frac{\partial \mathcal{D}}{\partial t}-\nabla \times \mathcal{H} & =-\mathcal{J}, \\
\nabla \cdot \mathcal{B} & =0,
\end{aligned}
$$

where

$\mathcal{E}=$ electric field,

$\mathcal{H}=$ magnetic field, 
$\mathcal{D}=$ electric displacement

$\mathcal{B}=$ magnetic induction,

$\rho=$ electric charge density,

$\mathcal{J}=$ electric current density.

Equation 2.1a) is called Faraday's law of induction, relating an electric field to a timevarying magnetic flux. The divergence condition (2.1b) is Gauss's law and gives the effect of the charge density on the electric displacement. The next equation, (2.1c), is Ampere's law relating magnetic fields and currents, which was extended by Maxwell to include induction of a magnetic field by a time-varying electric displacement. Finally, equation (2.1d) is Coulomb's law for magnetic flux, expressing the absence of isolated magnetic charges.

In this work, we only consider the classical time-harmonic problem. For a detailed overview of the investigation and derivation of this problem, we refer to [28].

\subsection{Time-harmonic Maxwell's equations}

Assume the vector fields $\mathcal{E}, \mathcal{D}, \mathcal{H}$, and $\mathcal{B}$ are periodic in time such that we can apply Fourier transform in time, i.e. of the form

$$
\mathcal{X}(x, t)=\Re\left(e^{-i \omega t} \mathbf{X}(x)\right) .
$$

The partial derivative to $t$ will become a multiplication, e.g., $\partial_{t} \mathcal{X}=-i \omega \mathcal{X}$. Here $\omega$ is a positive constant called the frequency. To stay consistent we assume also the charge density $\rho$, as well as the current density $\mathcal{J}$ to be of the form $(2.2)$. 
Under this assumption on the principle variables in 2.1], one obtains the timeharmonic Maxwell equations,

$$
\begin{array}{r}
-i \omega \mathbf{B}+\nabla \times \mathbf{E}=0, \\
\nabla \cdot \mathbf{D}=\rho, \\
-i \omega \mathbf{D}-\nabla \times \mathbf{H}=-\mathbf{J}, \\
\nabla \cdot \mathbf{B}=0 .
\end{array}
$$

The electric and magnetic flux densities $\mathbf{D}, \mathbf{B}$ are related to the field intensities $\mathbf{E}, \mathbf{H}$ via the so-called constitutive relations,

$$
\mathbf{D}=\epsilon \mathbf{E} \quad \text { and } \quad \mathbf{B}=\mu \mathbf{H},
$$

where $\epsilon$ is the permittivity and $\mu$ is the permeability. Furthermore, in conducting materials the electric field induces a conduction current, which is given by Ohm's law

$$
\mathbf{J}=\sigma \mathbf{E}+\mathbf{J}_{a}
$$

where $\sigma$ is called the conductivity and is a non-negative function of position. The vector function $\mathbf{J}_{a}$ describes the applied current density. In a vacuum (free space), the permeability, the permittivity and conductivity are,

$$
\mu_{0}=4 \pi \times 10^{-7} \mathrm{Vs} /(\mathrm{Am}), \quad \epsilon_{0}=8.854 \times 10^{-12} \mathrm{~F} / \mathrm{m}, \quad \text { and } \quad \sigma_{0}=0 .
$$

The free space permeability and permittivity are further connected by $c_{0}=1 / \sqrt{\epsilon_{0} \mu_{0}}$, where $c_{0} \approx 2.998 \times 10^{8} \mathrm{~m} / \mathrm{s}$ is the free space speed of light. 
Using the constitutive relations in (2.4) and (2.5), we can obtain Maxwell's Equations with only electric and magnetic fields:

$$
\begin{aligned}
\nabla \times \mathbf{E}-i \omega \mu \mathbf{H} & =0 \\
\nabla \times \mathbf{H}+(i \omega \epsilon-\sigma) \mathbf{E} & =\mathbf{J}_{a} \\
\nabla \cdot(\epsilon \mathbf{E}) & =\rho, \\
\nabla \cdot(\mu \mathbf{H}) & =0 .
\end{aligned}
$$

Using 2.6a to eliminate $\mathbf{H}$ from 2.6b yields

$$
\nabla \times\left(\frac{1}{i \omega \mu} \nabla \times \mathbf{E}\right)+(i \omega \epsilon-\sigma) \mathbf{E}=\mathbf{J}_{a}
$$

Usually, one writes this equation in a slightly different way by introducing the dimensionless, relative permittivity and permeability by

$$
\mu_{r}=\frac{\mu}{\mu_{0}}, \quad \epsilon_{r}=\frac{\bar{\beta}}{\epsilon_{0}}, \quad \bar{\beta}=\epsilon+\frac{i \sigma}{\omega} .
$$

Then equation (2.7) takes the form

$$
\nabla \times\left(\mu_{r}^{-1} \nabla \times \mathbf{E}\right)-\kappa^{2} \epsilon_{r} \mathbf{E}=i \omega \mu_{0} \mathbf{J}_{a}
$$

where the wavenumber $\kappa=\omega \sqrt{\mu_{0} \epsilon_{0}}$. We shall generally use 2.8 in this thesis.

\subsection{Strong Form (curl-curl problem)}

Let $\Omega \subset \mathbb{R}^{3}$ be a bounded and Lipschitz domain with connected boundary $\partial \Omega$ and unit outward normal n. We consider solving the classical time-harmonic Maxwell's equations subject to a perfect conducting boundary condition as follows:

$$
\nabla \times\left(\mu^{-1} \nabla \times \mathbf{u}\right)-\omega^{2} \beta \mathbf{u}=\mathbf{f} \text { in } \Omega \quad, \quad \mathbf{u} \times \mathbf{n}=\mathbf{0} \text { on } \partial \Omega
$$


where $\mathbf{u}=\mathbf{E}$ is the time-harmonic electric field corresponding to a given current density $\mathbf{f}=i \omega \mathbf{J}_{a}, \beta=\epsilon_{0} \epsilon_{r}$ and $\mu=\mu_{r} \mu_{0}$. Although we have chosen homogeneous Dirichlet boundary conditions in (2.9), and will focus on this case in our work, some of our results can easily be generalized to other types of boundary conditions.

\subsection{Variational Framework}

In this section we derive a variational (weak) formulation of the curl-curl problem (2.9). In order to pose this variational form, we must first define appropriate function spaces for the problem under consideration.

\subsubsection{Notation}

The gradient of a scalar-valued function of three variables $q=\left(q_{1}, q_{2}, q_{3}\right)$ is given by

$$
\operatorname{grad} q=\nabla q=\left(\frac{\partial q}{\partial q_{1}}, \frac{\partial q}{\partial q_{2}}, \frac{\partial q}{\partial q_{3}}\right)
$$

For a vector $\mathbf{v}=\left(v_{1}, v_{2}, v_{3}\right)$ the formulas for the divergence and curl are

$$
\begin{aligned}
& \operatorname{div} \mathbf{v}=\nabla \cdot \mathbf{v}=\frac{\partial v_{1}}{\partial x}+\frac{\partial v_{2}}{\partial y}+\frac{\partial v_{3}}{\partial z} \\
& \operatorname{curl} \mathbf{v}=\nabla \times \mathbf{v}=\left(\frac{\partial v_{3}}{\partial y}-\frac{\partial v_{2}}{\partial z}, \frac{\partial v_{1}}{\partial z}-\frac{\partial v_{3}}{\partial x}, \frac{\partial v_{2}}{\partial x}-\frac{\partial v_{1}}{\partial y}\right)
\end{aligned}
$$

\subsubsection{Function Spaces}

The following function spaces will turn out to provide a natural setting for the investigation of the PDEs discussed above and ensure that all expressions in variational form are well-defined. 
Definition 2.1 (Function Spaces). We define the spaces

$$
\begin{aligned}
L^{2}(\Omega) & =\left\{u: \int_{\Omega} u^{2} d V<\infty\right\}, \\
H^{1}(\Omega) & =\left\{p \in L^{2}(\Omega): \nabla p \in\left[L^{2}(\Omega)\right]^{3}\right\}, \\
H(\operatorname{curl} ; \Omega) & =\left\{\mathbf{u} \in\left[L^{2}(\Omega)\right]^{3}: \nabla \times \mathbf{u} \in\left[L^{2}(\Omega)\right]^{3}\right\}, \\
H(\operatorname{div} ; \Omega) & =\left\{\mathbf{p} \in\left[L^{2}(\Omega)\right]^{3}: \nabla \cdot \mathbf{p} \in\left[L^{2}(\Omega)\right]^{3}\right\},
\end{aligned}
$$

and the corresponding norms and semi-norms

$$
\begin{array}{rlrl}
\|u\|_{L^{2}(\Omega)} & =\left(\int_{\Omega}|u|^{2} d V\right)^{1 / 2}, \\
\|p\|_{H^{1}(\Omega)} & =\left(\|p\|_{L^{2}(\Omega)}^{2}+\|\nabla p\|_{L^{2}(\Omega)}^{2}\right)^{1 / 2}, & |p|_{H^{1}(\Omega)}=\|\nabla p\|_{L^{2}(\Omega)}, \\
\|\mathbf{u}\|_{H(\operatorname{curl} ; \Omega)} & =\left(\|\mathbf{u}\|_{L^{2}(\Omega)}^{2}+\|\nabla \times \mathbf{u}\|_{L^{2}(\Omega)}^{2}\right)^{1 / 2}, & |\mathbf{u}|_{H(\operatorname{curl} ; \Omega)}=\|\nabla \times \mathbf{u}\|_{L^{2}(\Omega)}, \\
\|\mathbf{p}\|_{H(\operatorname{div} ; \Omega)} & =\left(\|\mathbf{p}\|_{L^{2}(\Omega)}^{2}+\|\nabla \cdot \mathbf{p}\|_{L^{2}(\Omega)}^{2}\right)^{1 / 2}, & |\mathbf{p}|_{H(\operatorname{div} ; \Omega)}=\|\nabla \cdot \mathbf{p}\|_{L^{2}(\Omega)} .
\end{array}
$$

All the above spaces are Hilbert spaces when equipped with the corresponding scalar products defined by,

$$
\begin{aligned}
(u, v)_{L^{2}(\Omega)} & =\int_{\Omega} u \bar{v} d V \\
(p, q)_{H^{1}(\Omega)} & =\int_{\Omega} \nabla p \cdot \nabla \bar{q} d V+\int_{\Omega} p \bar{q} d V \\
(\mathbf{u}, \mathbf{v})_{H(\operatorname{curl} ; \Omega)} & =\int_{\Omega}(\nabla \times \mathbf{u}) \cdot(\nabla \times \overline{\mathbf{v}}) d V+\int_{\Omega} \mathbf{u} \cdot \overline{\mathbf{v}} d V \\
(\mathbf{p}, \mathbf{q})_{H(\operatorname{div} ; \Omega)} & =\int_{\Omega}(\nabla \cdot \mathbf{p})(\nabla \cdot \overline{\mathbf{q}}) d V+\int_{\Omega} \mathbf{p} \cdot \overline{\mathbf{q}} d V
\end{aligned}
$$

Over-bar here (e.g. $\bar{v}$ ) denotes complex conjugation. The relation between the functional spaces through their differential operators can be summarized in the de Rham diagram

$$
H^{1}(\Omega) \stackrel{\nabla}{\longrightarrow} H(\operatorname{curl} ; \Omega) \stackrel{\nabla \times}{\longrightarrow} H(\operatorname{div} ; \Omega) \stackrel{\nabla \cdot}{\longrightarrow} L_{2}(\Omega)
$$


From the point of view of Maxwell's equations the space $H(\operatorname{curl} ; \Omega)$ is of central importance since it corresponds to the space of finite-energy solutions.

\subsubsection{Traces, Boundary Conditions and Variational Formulation}

If $H(\operatorname{curl} ; \Omega)$ is to be used as the energy space for Maxwell's equations, then tangential trace of functions in this space must be well-defined $9,10,28$. To show that they are, we first define boundary trace operators for smooth functions $u \in \mathrm{C}^{\infty}(\bar{\Omega})$ and $\mathbf{u} \in\left[\mathcal{C}^{\infty}(\bar{\Omega})\right]^{3}:$

$$
\gamma_{0}(u)=\left.u\right|_{\partial \Omega} \quad, \quad \gamma_{t}(\mathbf{u})=\mathbf{n} \times\left.\mathbf{u}\right|_{\partial \Omega} \quad, \quad \gamma_{T}(\mathbf{u})=\left(\mathbf{n} \times\left.\mathbf{u}\right|_{\partial \Omega}\right) \times \mathbf{n} .
$$

It is well-known that $\gamma_{0}$ can be continuously extended to a bounded linear operator on $H^{1}(\Omega)$, and this fact is used to define the trace space $H^{1 / 2}(\partial \Omega)$, and its norm

$$
H^{1 / 2}(\partial \Omega)=\gamma_{0}\left(H^{1}(\Omega)\right) \quad, \quad\|v\|_{H^{1 / 2}(\partial \Omega)}=\inf _{\substack{w \in H^{1}(\Omega) \\ \gamma_{0}(w)=v}}\|w\|_{H^{1}(\Omega)} .
$$

We define $H^{-1 / 2}(\partial \Omega)=\left[H^{1 / 2}(\partial \Omega)\right]^{\prime}$, the dual space of $H^{1 / 2}(\partial \Omega)$ (see Section 2.4.4). Similarly, the tangential traces $\gamma_{t}$ and $\gamma_{T}$ can be continuously extended to bounded linear operators on $H(\operatorname{curl} ; \Omega)$, as discussed below.

Lemma 2.2. (Integration by Parts). The tangential trace operator $\gamma_{t}$ is a bounded linear operator from $H(\operatorname{curl} ; \Omega)$ into $\left[H^{-1 / 2}(\partial \Omega)\right]^{3}$, and it holds

$$
\int_{\Omega}(\nabla \times \mathbf{u}) \cdot \overline{\mathbf{v}} d V-\int_{\Omega} \mathbf{u} \cdot(\nabla \times \overline{\mathbf{v}}) d V=\int_{\partial \Omega} \gamma_{t}(\mathbf{u}) \cdot \overline{\mathbf{v}} d s
$$

for any $\mathbf{u} \in H(\operatorname{curl} ; \Omega)$ and $\mathbf{v} \in\left[H^{1}(\Omega)\right]^{3}$

Proof of this lemma can be found in [10].

Remark 2.3. The trace $\gamma_{t}$ from $H(\operatorname{curl} ; \Omega)$ into $\left[H^{-1 / 2}(\partial \Omega)\right]^{3}$ is not surjective since in 2.11$)\left[H^{-1 / 2}(\partial \Omega)\right]^{3}$ contains vectors that are not tangential to $\partial \Omega$. The correct space of the trace is examined in the next theorem. 
Define a Banach space

$Y(\partial \Omega)=\left\{\mathbf{f} \in\left[H^{-1 / 2}(\partial \Omega)\right]^{3} \mid\right.$ there exists $\mathbf{u} \in H(\operatorname{curl} ; \Omega)$ such that $\left.\gamma_{t}(\mathbf{u})=\mathbf{f}\right\}$

with norm given by

$$
\|\mathbf{f}\|_{Y(\partial \Omega)}=\inf _{\substack{\mathbf{u} \in H(\operatorname{curl} ; \Omega) \\ \gamma_{t}(\mathbf{u})=\mathbf{f}}}\|\mathbf{u}\|_{H(\operatorname{curl} ; \Omega)} .
$$

Theorem 2.4. The following statements hold:

1. $Y(\partial \Omega)$ is a Hilbert space.

2. $\gamma_{t}: H(\operatorname{curl} ; \Omega) \rightarrow Y(\partial \Omega)$ is surjective.

3. $\gamma_{T}: H(\operatorname{curl} ; \Omega) \rightarrow Y(\partial \Omega)^{\prime}$ is well-defined.

4. For any $\mathbf{u}, \mathbf{v} \in H(\operatorname{curl} ; \Omega)$, it holds

$$
\int_{\Omega}(\nabla \times \mathbf{u}) \cdot \overline{\mathbf{v}} d V-\int_{\Omega} \mathbf{u} \cdot(\nabla \times \overline{\mathbf{v}}) d V=\int_{\partial \Omega} \gamma_{t}(\mathbf{u}) \cdot \overline{\gamma_{T}(\mathbf{v})} d s
$$

$Y(\partial \Omega)^{\prime}$ is the dual space of $Y(\partial \Omega)$ (see Section 2.4.4). More details can be found in $9,10,28$.

The space $H_{0}(\operatorname{curl} ; \Omega)$ is equal to the space of $H(\operatorname{curl} ; \Omega)$ functions with homogenous tangential boundary conditions, and can be defined as

$$
H_{0}(\operatorname{curl} ; \Omega)=\left\{\mathbf{u} \in H(\operatorname{curl} ; \Omega): \gamma_{t}(\mathbf{u})=\mathbf{0} \text { on } \partial \Omega\right\}
$$

Now we ready to derive the variational formulation of the strong problem (curl-curl problem). We begin by multiplying problem (2.9) by a suitable "test" function $\mathbf{v}$, and integrating over $\Omega$, to obtain

$$
\int_{\Omega} \nabla \times\left(\mu^{-1} \nabla \times \mathbf{u}\right) \cdot \overline{\mathbf{v}} d V-\int_{\Omega} \omega^{2} \beta \mathbf{u} \cdot \overline{\mathbf{v}} d V=\int_{\Omega} \mathbf{f} \cdot \overline{\mathbf{v}} d V
$$


Using integration by parts to remove the outer curl operator $\mu^{-1} \nabla \times \mathbf{u}$ from the first integral, we obtain

$$
\int_{\Omega} \nabla \times\left(\mu^{-1} \nabla \times \mathbf{u}\right) \cdot \overline{\mathbf{v}} d V=\int_{\Omega}\left(\mu^{-1} \nabla \times \mathbf{u}\right) \cdot(\nabla \times \overline{\mathbf{v}}) d V+\int_{\partial \Omega} \gamma_{t}(\mathbf{u}) \cdot \overline{\gamma_{T}(\mathbf{v})} d V
$$

Thus, we have

$$
\int_{\Omega}\left(\mu^{-1} \nabla \times \mathbf{u}\right) \cdot(\nabla \times \overline{\mathbf{v}}) d V-\int_{\Omega} \omega^{2} \beta \mathbf{u} \cdot \overline{\mathbf{v}} d V=\int_{\Omega} \mathbf{f} \cdot \overline{\mathbf{v}} d V-\int_{\partial \Omega} \gamma_{t}(\mathbf{u}) \cdot \overline{\gamma_{T}(\mathbf{v})} d s
$$

By including our boundary conditions, we have the following weak form of the problem 2.9):

Find $\mathbf{u} \in \mathcal{H}$ such that

$$
B(\mathbf{u}, \mathbf{v})=F(\mathbf{v}) \text { for all } \mathbf{v} \in \mathcal{H}
$$

where $\mathcal{H}=H_{0}(\operatorname{curl} ; \Omega)$. The bilinear form $\mathrm{B}$ is given by

$$
B(\mathbf{u}, \mathbf{v})=\int_{\Omega}\left(\mu^{-1} \nabla \times \mathbf{u}\right) \cdot \nabla \times \overline{\mathbf{v}}-\omega^{2} \beta \mathbf{u} \cdot \overline{\mathbf{v}} d V
$$

and the linear form $F(\mathbf{v})$ is given by

$$
F(\mathbf{v})=\int_{\Omega} \mathbf{f} \cdot \overline{\mathbf{v}} d V
$$

In later sections, we may "abuse" notation by dropping the boundary trace notation in favor of more familiar quantities that are well-defined when the functions are sufficiently regular, e.g.

$$
\begin{aligned}
\int_{\partial \Omega} \gamma_{t}(\mathbf{u}) \cdot \overline{\gamma_{T}(\mathbf{v})} d s & =\int_{\partial \Omega}(\mathbf{n} \times \mathbf{u}) \cdot(\mathbf{n} \times \overline{\mathbf{v}} \times \mathbf{n}) d s \\
& =\int_{\partial \Omega}(\mathbf{u} \times \overline{\mathbf{v}}) \cdot \mathbf{n} d s=-\int_{\partial \Omega}(\mathbf{u} \times \mathbf{n}) \cdot \overline{\mathbf{v}} d s
\end{aligned}
$$

Here we have used: 
- the decomposition of $\mathbf{v}$ into its normal and tangential components, $\mathbf{v}=(\mathbf{v}$. $\mathbf{n}) \mathbf{n}+\mathbf{n} \times \mathbf{v} \times \mathbf{n}$,

- the fact $\mathbf{n} \times \mathbf{u}$ is orthogonal to $\mathbf{n}$, so $(\mathbf{n} \times \mathbf{u}) \cdot(\mathbf{n} \times \overline{\mathbf{v}} \times \mathbf{n})=\mathbf{n} \times \mathbf{u} \cdot \overline{\mathbf{v}}$,

- identities for reordering terms in a scalar triple product, $\mathbf{n} \times \mathbf{u} \cdot \overline{\mathbf{v}}=\mathbf{u} \times \overline{\mathbf{v}} \cdot \mathbf{n}=$ $-\mathbf{u} \times \mathbf{n} \cdot \overline{\mathbf{v}}$.

\subsubsection{Abstract Well-posedness Results for Variational Problems}

In this section, we briefly recall two key well-posedness results for variational problem such as 2.12). Before stating these results, we need the following definitions.

Let $X$ and $Y$ denote Hilbert-spaces provided with the scalar products $(\cdot, \cdot)_{X}$, $(\cdot, \cdot)_{Y}$, and the induced norms are denoted by $\|\cdot\|_{X}$ and $\|\cdot\|_{Y}$.

Definition 2.5. Let $X$ be a Hilbert space. A bounded linear functional on $X$ is a linear functional $T$ such that there exists an $M>0$ where $|T(x)| \leqslant M\|x\|$ for every $x \in X$. The dual space of $X$ is denoted by $X^{\prime}$ and defined to be the space of bounded linear functionals on $X$. If $f \in X^{\prime}$ then the norm of $f$ is

$$
\|f\|_{X^{\prime}}=\sup _{\substack{x \in X \\ x \neq 0}} \frac{|f(x)|}{\|x\|_{X}}
$$

We define the dual pairing $\langle\cdot, \cdot\rangle_{X^{\prime}, X}$ so that $\langle f, x\rangle_{X^{\prime}, X}=f(x)$ for all $x \in X$ and $f \in X^{\prime}$.

Definition 2.6. A mapping $a(\cdot, \cdot): X \times Y \rightarrow \mathbb{C}$ is called

1. a sesquilinear form if

$$
\begin{aligned}
& \text { - } a\left(\alpha_{1} u+\alpha_{2} v, \phi\right)=\alpha_{1} a(u, \phi)+\alpha_{2} a(v, \phi) \text { for all } \alpha_{1}, \alpha_{2} \in \mathbb{C}, u, v \in X \text { and } \\
& \phi \in Y
\end{aligned}
$$


- $a\left(u, \beta_{1} \phi+\beta_{2} \psi\right)=\overline{\beta_{1}} a(u, \phi)+\overline{\beta_{2}} a(u, \psi)$ for all $\beta_{1}, \beta_{2} \in \mathbb{C}, u \in X$ and $\phi, \psi \in Y$

2. bounded if there is a constant $C$ independent of $u \in X$ and $\phi \in Y$ such that

$$
|a(u, \phi)| \leqslant C\|u\|_{X}\|\phi\|_{Y} \quad \text { for all } \quad u \in X \text { and } \phi \in Y
$$

3. coercive if there is a constant $\alpha>0$ independent of $u \in X$ such that

$$
|a(u, u)| \geqslant \alpha\|u\|_{X}^{2} \quad \text { for all } \quad u \in X
$$

We now consider the abstract variational problem: Find $u \in X$ such that

$$
a(u, v)=f(v) \quad \text { for all } \quad v \in X
$$

where $f \in X^{\prime}$ is a given linear functional and $a(\cdot, \cdot)$ is a bounded coercive sesquilinear form on $X \times X$. The following lemma guarantees the existence and uniqueness of this variational problem.

Lemma 2.7. (Lax-Milgram) Suppose $a: X \times X \rightarrow \mathbb{C}$ is a bounded and coercive sesquilinear form. Then for each $f \in X^{\prime}$ there exists a unique solution $u \in X$ to 2.15, and

$$
\|u\|_{X} \leqslant \frac{\|f\|_{X^{\prime}}}{\alpha}
$$

In the problem that we consider in this study, the presence of the term $-\omega^{2} \beta \mathbf{u} \cdot \overline{\mathbf{v}}$ in $B(\mathbf{u}, \mathbf{v})$ typically means that it is not coercive on $\mathcal{H}$, so the Lax-Milgram lemma is not sufficient to establish well-posedness of (2.12). To counter this problem, we need a further generalization of Max-Milgram lemma.

Theorem 2.8. (Generalized Lax-Milgram Lemma) Let $a(\cdot, \cdot): X \times Y \rightarrow \mathbb{C}$ be a bounded sesquilinear form which has the following properties: 
1. There is a constant $\alpha>0$ such that

$$
\inf _{u \in X} \sup _{v \in Y} \frac{|a(u, v)|}{\|u\|_{X}\|v\|_{Y}} \geqslant \alpha
$$

2. For every $v \in X, v \neq 0$

$$
\sup _{u \in X}|a(u, v)|>0
$$

Then, for each $f \in X^{\prime}$, there exists a unique solution $u \in X$ to 2.15 and,

$$
\|u\|_{X} \leqslant \frac{\|f\|_{X^{\prime}}}{\alpha}
$$

In [28], Monk provides some sufficient conditions for the well-posedness of 2.12 in terms of the parameters $\mu, \beta, \omega$ and the function $\mathbf{f}$ on some (connected, Lipschitz) partition of $\Omega$ :

1. the parameters $\mu, \beta \in L^{\infty}(\Omega)$ are piecewise smooth and $\mu, \beta>0$ almost everywhere on $\Omega$.

2. $\Im(\beta)=0$ on $\Omega$ and $\omega^{2}$ is not an eigenvalue of the eigenvalue problem

$$
\int_{\Omega}\left(\mu^{-1} \nabla \times \mathbf{u}\right) \cdot \nabla \times \overline{\mathbf{v}}=\omega^{2} \int_{\Omega} \beta \mathbf{u} \cdot \overline{\mathbf{v}} d V \text { for all } \mathbf{v} \in \mathcal{H}
$$

3. $\mathbf{f} \in\left(L^{2}(\Omega)\right)^{3}$.

The boundedness of $B$ and $F$ are straight-forward to verify, and we do so below. A proof that $B$ satisfies the inf-sup conditions under these assumptions on the data 
is given in 28$]$.

$$
\begin{aligned}
|B(\mathbf{u}, \mathbf{v})| & \leqslant\left\|\mu^{-1}\right\|_{L^{\infty}(\Omega)}\|\nabla \times \mathbf{u}\|_{L^{2}(\Omega)}\|\nabla \times \mathbf{v}\|_{L^{2}(\Omega)}+\omega^{2}\|\beta\|_{L^{\infty}(\Omega)}\|\mathbf{u}\|_{L^{2}(\Omega)}\|\mathbf{v}\|_{L^{2}(\Omega)}, \\
& \leqslant \max \left\{\left\|\mu^{-1}\right\|_{L^{\infty}(\Omega)}, \omega^{2}\|\beta\|_{L^{\infty}(\Omega)}\right\}\left(\begin{array}{c}
\|\nabla \times \mathbf{u}\|_{L^{2}(\Omega)} \\
\|\mathbf{u}\|_{L^{2}(\Omega)}
\end{array}\right) \cdot\left(\begin{array}{c}
\|\nabla \times \mathbf{v}\|_{L^{2}(\Omega)} \\
\|\mathbf{v}\|_{L^{2}(\Omega)}
\end{array}\right) \\
& \leqslant M\left(\|\mathbf{u}\|_{L^{2}(\Omega)}^{2}+\|\nabla \times \mathbf{u}\|_{L^{2}(\Omega)}^{2}\right)^{1 / 2}\left(\|\mathbf{v}\|_{L^{2}(\Omega)}^{2}+\|\nabla \times \mathbf{v}\|_{L^{2}(\Omega)}^{2}\right)^{1 / 2} \\
& =M\|\mathbf{u}\|_{H(\operatorname{curl} ; \Omega)}\|\mathbf{v}\|_{H(\operatorname{curl} ; \Omega)} .
\end{aligned}
$$

where $M=\max \left\{\left\|\mu^{-1}\right\|_{L^{\infty}(\Omega)}, \omega^{2}\|\beta\|_{L^{\infty}(\Omega)}\right\}$.

Again using the Cauchy-Schwarz inequality with $F(\mathbf{v})$, we have

$$
|F(\mathbf{v})| \leqslant\|\mathbf{f}\|_{L^{2}(\Omega)}\|\mathbf{v}\|_{L^{2}(\Omega)} \leqslant\|\mathbf{f}\|_{L^{2}(\Omega)}\|\mathbf{v}\|_{H(\operatorname{curl} ; \Omega)} .
$$

Remark 2.9. From here onwards we shall absorb the constants $\omega$ and $\beta$ in one constant, and we will call it $\beta$ again.

Remark 2.10. In our particular case, the regular inf-sup conditions is defined by

$$
\inf _{\substack{\mathbf{u} \in \mathcal{H} \\ \mathbf{u} \neq 0}} \sup _{\substack{\mathbf{v} \in \mathcal{H} \\ \mathbf{v} \neq 0}} \frac{|B(\mathbf{u}, \mathbf{v})|}{\|\mathbf{u}\|_{\mathcal{H}}\|\mathbf{v}\|_{\mathcal{H}}} \geqslant \tilde{\alpha} .
$$

for some constant $\tilde{\alpha}>0$. 


\section{Chapter 3}

\section{Finite Elements for Maxwell's Equations}

From the previous chapter, we saw that the Sobolev space $H(\operatorname{curl} ; \Omega)$ plays a central role in the variational theory of Maxwell's equations. Thus we need suitable finite elements in this space for discretizing the Maxwell system. First, we will shortly recall the main ingredients for the finite element method (FEM), and then define the classical finite element spaces (FE spaces) for (2.12), namely the Nédélec elements of first kind. lastly, we will introduce the discrete variational problem.

\subsection{Triangulation and Finite Element Spaces}

The first step in applying finite elements methods is to generate a finite element triangulation (mesh) covering the domain $\Omega$. Recall that $\Omega$ is a bounded polyhedral domain with Lipschitz boundary.

Definition 3.1. A triangulation (mesh) $\mathcal{T}$ is a finite non-overlapping subdivision of $\Omega$ into elements $T_{i}$ of simple geometry. A triangulation is called regular, if

1. the elements are non-overlapping, i.e.

$$
\operatorname{interior}\left(T_{i}\right) \cap \text { interior }\left(T_{j}\right)=\varnothing \quad \text { for } \quad i \neq j
$$

2. the triangulation $\mathcal{T}$ is a covering of $\Omega$, i.e.

$$
\bigcup_{T_{i} \in \mathcal{T}} T_{i}=\bar{\Omega}
$$

3. the intersection $T_{i} \cap T_{j}$ of two different elements $(i \neq j)$ is either empty, or a vertex, or an edge or a face of both elements; 
4. each $T \in \mathcal{T}$ is a Lipschitz domain.

For each face $F$, we define the parameter $h_{F}$ such that

$$
h_{F}=\text { diameter of } F \text {, }
$$

and for each element $T$, we define the parameter $h_{T}$ such that

$$
\left.h_{T}=\text { diameter of } T \text { (diameter of the smallest sphere containing } \bar{T}\right) \text {, }
$$

then $h=\max _{T \in \mathcal{T}} h_{T}$ so that the index $h$ denotes the maximum diameter of the elements $T \in \mathcal{T}$.

We will frequently use the following notations

$$
\begin{array}{lll}
\text { the set of vertices } & \mathcal{V}=\left\{z_{i}\right\}, \quad \text { the set of edges } & \mathcal{E}=\left\{e_{i}\right\}, \\
\text { the set of faces } & \mathcal{F}=\left\{F_{i}\right\}, \quad \text { the set of elements } & \mathcal{T}=\left\{T_{i}\right\} .
\end{array}
$$

The sets of the interior faces and interior edges are denoted by $\mathcal{F}_{I}$ and $\mathcal{E}_{I}$, respectively, and the (local) sets of vertices, edges, and faces belonging to the element $T$ are denoted by $\mathcal{V}_{T}, \mathcal{E}_{T}$, and $\mathcal{F}_{T}$.

In this study, we consider a tetrahedral mesh of a polyhedral domain. Thus each $T \in \mathcal{T}$ is a tetrahedron with vertices $z_{1}, z_{2}, z_{3}, z_{4} \in \mathcal{V}$.

After having the most characteristic aspect of the finite element method, the triangulation, we can define a finite element. Following the classical approach of Ciarlet in [12], the classical definition is the following:

Definition 3.2 (Finite Element). The triple $\left(T, \mathcal{P}_{T}, \Sigma_{T}\right)$ is a finite element provided,

1. $T \subset \mathbb{R}^{n}$ is a compact set with non-empty interior and (piecewise smooth) Lipschitz boundary,

2. $\mathcal{P}_{T}$ is a finite-dimensional space of functions on $T$, with $\operatorname{dim}\left(\mathcal{P}_{T}\right)=k$. 
3. The functionals $\Sigma_{T}=\left\{f_{i, T}\right\}_{i=1}^{k}$ are a basis for $\mathcal{P}_{T}$. They are called the (local) degrees of freedom of the finite element.

\subsection{Polynomial spaces and Nédélec Space}

Local FE spaces are usually spanned by polynomial functions. Let us start by defining basic polynomial spaces that will be needed in the forthcoming definitions. We define $\mathbb{P}_{p}$ to be the polynomials of (total) degree at most $p$ in $x=\left(x_{1}, x_{2}, x_{3}\right)$ and $\tilde{\mathbb{P}}_{p}$ to be the subset of $\mathbb{P}_{p}$ consisting of homogeneous polynomials of degree precisely $p$. Recall that a polynomial $v$ is called homogeneous of degree $p$ when $v(\alpha x)=\alpha^{p} v(x)$ for any constant $\alpha$-in other words, all monomial terms in $v$ have degree $p$. It holds that

$$
\operatorname{dim} \mathbb{P}_{p}=\left(\begin{array}{c}
p+3 \\
3
\end{array}\right) \quad, \quad \operatorname{dim} \tilde{\mathbb{P}}_{p}=\left(\begin{array}{c}
p+2 \\
2
\end{array}\right)
$$

More generally, if these polynomials had been in $n$ variables instead of 3 , then 3 would be replaced by $n$ and 2 by $n-1$ in $(3.1)$. A nice algebraic property of $\tilde{\mathbb{P}}_{p}$ is that

$$
v=(x \cdot \nabla v) / p \text { for } v \in \tilde{\mathbb{P}}_{p}, \text { so }(\nabla v=\mathbf{0} \Longrightarrow v=0) \text { for } v \in \tilde{\mathbb{P}}_{p}
$$

We also see that $\nabla v \in\left[\tilde{\mathbb{P}}_{p-1}\right]^{3}$ for $v \in \tilde{\mathbb{P}}_{p}$.

We now define a space $\mathcal{S}_{p}$ and give its dimension,

$$
\mathcal{S}_{p}=\left\{\mathbf{v} \in\left[\tilde{\mathbb{P}}_{p}\right]^{3}: x \cdot \mathbf{v}=0\right\} \quad, \quad \operatorname{dim} \mathcal{S}_{p}=3 \operatorname{dim} \tilde{\mathbb{P}}_{p}-\operatorname{dim} \tilde{\mathbb{P}}_{p+1}=p(p+2)
$$

Since $v \in \tilde{\mathbb{P}}_{p+1}$ if and only if $v=x \cdot \mathbf{v}$ for some $\mathbf{v} \in\left[\tilde{\mathbb{P}}_{p}\right]^{3}$ (in fact $v=(x \cdot \nabla v) /(p+1)$ ), the constraint $x \cdot \mathbf{v}=0$ in the definition of $\mathcal{S}_{p}$ removes a subspace of dimension $\operatorname{dim} \tilde{\mathbb{P}}_{p+1}$ from $\left[\tilde{\mathbb{P}}_{p}\right]^{3}$; this explains the formula for $\operatorname{dim} \mathcal{S}_{p}$. It also follows from the discussion above that $\mathcal{S}_{p}$ is gradient free, in the sense that $\mathbf{v}=\nabla v \in \mathcal{S}_{p}$ if and only if $\mathbf{v}=\mathbf{0}$ 
Nédélec spaces are perhaps the most widely used finite element spaces in computational electromagnetic. For discretizing the variational problem (2.12) we use the $H(\operatorname{curl}, \Omega)$-conforming finite element space introduced by Nédélec [29]. For any given conforming tetrahedral triangulation $\mathcal{T}$ of $\Omega$, the local $p$-order Nédélec space of the first-kind, with its dimension, is defined by

$$
\mathcal{R}_{p}=\left[\mathbb{P}_{p-1}\right]^{3} \oplus \mathcal{S}_{p} \quad, \quad \operatorname{dim} \mathcal{R}_{p}=p(p+2)(p+3) / 2 .
$$

A key algebraic property of $\mathcal{R}_{p}$ is that

$$
\left[\mathbb{P}_{p}\right]^{3}=\mathcal{R}_{p} \oplus \nabla \tilde{\mathbb{P}}_{p+1}
$$

Applying (3.5) to 3.4, we have a decomposition of $\mathcal{R}_{p}$ into a gradient space and a gradient-free space,

$$
\begin{aligned}
\mathcal{R}_{p} & =\left(\nabla \tilde{\mathbb{P}}_{1} \oplus \cdots \oplus \nabla \tilde{\mathbb{P}}_{p}\right) \oplus\left(\mathcal{S}_{1} \oplus \cdots \oplus \mathcal{S}_{p}\right) \\
& =\nabla \mathbb{P}_{p} \oplus\left(\mathcal{S}_{1} \oplus \cdots \oplus \mathcal{S}_{p}\right)=\mathcal{R}_{p}^{G} \oplus \mathcal{R}_{p}^{F} .
\end{aligned}
$$

The dimensions of these spaces are

$$
\begin{aligned}
& \operatorname{dim} \mathcal{R}_{p}^{G}=\sum_{k=1}^{p} \operatorname{dim} \nabla \tilde{\mathbb{P}}_{k}=\sum_{k=1}^{p} \operatorname{dim} \tilde{\mathbb{P}}_{k}=\sum_{k=1}^{p}\left(\begin{array}{c}
k+2 \\
2
\end{array}\right)=p\left(p^{2}+6 p+11\right) / 6 \\
& \operatorname{dim} \mathcal{R}_{p}^{F}=\sum_{k=1}^{p} \operatorname{dim} \mathcal{S}_{k}=\sum_{k=1}^{p} k(k+2)=p(p+1)(2 p+7) / 6 .
\end{aligned}
$$

We also note that $\operatorname{dim} \mathcal{R}_{p}^{G}=\operatorname{dim} \nabla \mathbb{P}_{p}=\operatorname{dim} \mathbb{P}_{p}-1$. For reference, we include a table of these dimensions for a few small values of $p$ in Table 3.1 .

Now we can define the curl conforming element on a general tetrahedron $T$ and describe how $\mathbf{v} \in V_{p}(T)$ is uniquely determined by specifying certain moments on its edges, faces and volume.

Definition 3.3. The curl-conforming finite element of Nédélec is defined by: 


\begin{tabular}{|c||ccc|ccc|}
\hline$p$ & $\operatorname{dim} \mathcal{R}_{p}$ & $\operatorname{dim} \mathcal{R}_{p}^{G}$ & $\operatorname{dim} \mathcal{R}_{p}^{F}$ & $\operatorname{dim} V_{p}^{E}(T)$ & $\operatorname{dim} V_{p}^{F}(T)$ & $\operatorname{dim} V_{p}^{T}(T)$ \\
\hline 1 & 6 & 3 & 3 & 6 & 0 & 0 \\
2 & 20 & 9 & 11 & 12 & 8 & 0 \\
3 & 45 & 19 & 26 & 18 & 24 & 3 \\
\hline
\end{tabular}

Table 3.1: The dimensions of $V_{p}(T)=\mathcal{R}_{p}$ and its gradient and gradient-free subspaces $\mathcal{R}_{p}^{G}$ and $\mathcal{R}_{p}^{F}$, together with those of the edge, face and volume subspaces $V_{p}^{E}(T)$, $V_{p}^{F}(T), V_{p}^{T}(T)$, for small $p$.

- $T$ is a tetrahedron,

- $V_{p}(T)=\mathcal{R}_{p}$,

- The degrees of freedom are of three types associated with edges e of $T$, faces $F$ of $T$ and $T$ itself. We denote by $\mathbf{t}$ a unit vector in the direction of the edge $e$. There are three different degrees of freedom:

1. the first set is associated with edges of the element:

$$
M_{e}(\mathbf{v})=\left\{\int_{e} \mathbf{v} \cdot \mathbf{t} q d s \text { for all } q \in \mathbb{P}_{p-1}(e) \text { and } e \in \mathcal{E}_{T}\right\}
$$

2. the second set is associated with faces of the element:

$$
M_{F}(\mathbf{v})=\left\{\int_{F_{k}}\left(\mathbf{v} \times \mathbf{n}_{k}\right) \cdot \mathbf{q} d A \text { for all } \mathbf{q} \in\left[\mathbb{P}_{p-2}(F)\right]^{3} \text { and } F \in \mathcal{F}\right\}
$$

3. the last set is associated with the volume:

$$
M_{T}(\mathbf{v})=\left\{\int_{T} \mathbf{v} \cdot \mathbf{q} d V \text { for all } \mathbf{q} \in\left[\mathbb{P}_{p-3}(T)\right]^{3}\right\}
$$

Then $\Sigma_{T}=M_{e}(\mathbf{v}) \cup M_{F}(\mathbf{v}) \cup M_{T}(\mathbf{v})$.

In light of this, we decompose $V_{p}(T)$ as $V_{p}(T)=V_{p}^{E}(T) \oplus V_{p}^{F}(T) \oplus V_{p}^{T}(T)$, where all functions in $V_{p}^{T}(T)$ have vanishing face and edge moments, and $V_{p}^{F}(T) \subset V_{p}(T) \ominus$ 
$V_{p}^{T}(T)$ consists of those functions which have vanishing edge moments. We refer to $V_{p}^{E}(T)$ as the edge space, $V_{p}^{F}(T)$ as the face space, and $V_{p}^{T}(T)$ as the volume space.

\subsection{Barycentric Coordinates on the Tetrahedron}

Particularly important polynomials in $\mathbb{P}_{1}$ are the barycentric coordinates, defined below. It is useful to replace the Euclidean coordinates of the point $\mathbf{x} \in \mathbb{R}^{3}$ by barycentric coordinates denoted by $\lambda_{i}=\lambda_{i}(\mathbf{x})$ with respect to the four vertices $z_{i} \in$ $\mathcal{V}_{T}$, and many important finite elements can be expressed in terms of these coordinates as we will do.

Definition 3.4 (Barycentric Coordinates). Let $z_{i}, i=1, \cdots, 4$, be the vertices of a non-degenerate tetrahedron $T \subset \mathbb{R}^{3}$, we define the following:

- Six edges $e_{i j}, 1 \leqslant i<j \leqslant 4$, between vertices $z_{i}$ and $z_{j}$, and the unit tangent vector $\mathbf{t}_{i j}=\left(z_{j}-z_{i}\right) /\left|z_{j}-z_{i}\right|$.

- Four faces $F_{k}, 1 \leqslant k \leqslant 4$, with $F_{k}$ opposite vertex $z_{k}$, and outward unit normal $\mathbf{n}_{k}$. We define the distance between $z_{k}$ and the plane containing $F_{k}$ as $\alpha_{k}$, and note that $\alpha_{k}=3|T| /\left|F_{k}\right|$.

Then the barycentric coordinate function $\lambda_{i}(\mathbf{x})$ is the unique polynomial function in $\mathbb{P}_{1}$ such that $\lambda_{i}\left(\mathbf{x}_{j}\right)=\delta_{i j}, 1 \leqslant j \leqslant 4$. A formula for $\lambda_{i}\left(\mathbf{x}_{j}\right)$ is given by

$$
\lambda_{i}(x)=1-\frac{\left(x-z_{i}\right) \cdot \mathbf{n}_{i}}{\alpha_{i}}=\delta_{i j}+\nabla \lambda_{i} \cdot\left(x-z_{j}\right)
$$


An alternate formulation is found by solving the system of equations

$$
\sum_{k=1}^{4} \lambda_{k}(x) z_{k}=x \quad, \quad \sum_{k=1}^{4} \lambda_{k}(x)=1 \Longleftrightarrow \underbrace{\left(\begin{array}{cccc}
1 & 1 & 1 & 1 \\
z_{1} & z_{2} & z_{3} & z_{4}
\end{array}\right)}_{M}\left(\begin{array}{c}
\lambda_{1}(x) \\
\lambda_{2}(x) \\
\lambda_{3}(x) \\
\lambda_{4}(x)
\end{array}\right)=\left(\begin{array}{c}
1 \\
x
\end{array}\right) .
$$

Cramer's rule can be used to determine a generic formula for each coordinate, with $\operatorname{det}(M)= \pm 6|T|$. The assumption that the elements are non-degenerate implies that $|T| \neq 0$.

\subsection{Basis for Local Space $V_{p}(T)=\mathcal{R}_{p}$}

The decomposition $V_{p}(T)=V_{p}^{E}(T) \oplus V_{p}^{F}(T) \oplus V_{p}^{T}(T)$ suggests that a natural basis for $V_{p}(T)$ would also be decomposed in this manner. Two references in which this is accomplished in terms of barycentric coordinates are [17,35]. We essentially reproduce the basis given in [17] where the basis for Nédélec spaces are presented of any order in any space dimension. The main feature of this basis is that it is expressed just in terms of the barycentric coordinates of the simplex. Table 3.2 shows the basis for Nédélec space on a tetrahedron $T$ in barycentric coordinates for order $1 \leqslant p \leqslant 3$. We take the vertices of $T$ to be indexed by $(i, j, k, \ell)$, or $(1,2,3,4)$ if all four indices appear in a given expression.

\subsection{Global Spaces, Dimension and Bases}

Given a conforming tetrahedral partition $\mathcal{T}$ of $\Omega$, we define the global approximation space $V_{p}$ as:

$$
V_{p}=\left\{\mathbf{v} \in \mathcal{H}:\left.\mathbf{v}\right|_{T} \in V_{p}(T) \text { for all } T \in \mathcal{T}\right\}
$$




\begin{tabular}{|c||c|c|c|}
\hline$p$ & $V_{p}^{E}(T)$ on $e_{i j}$ & $V_{p}^{F}(T)$ on $F_{\ell}=F_{i j k}$ & $V_{p}^{T}(T)$ \\
\hline 1 & $\lambda_{i} \nabla \lambda_{j}-\lambda_{j} \nabla \lambda_{i}$ & & \\
\hline 2 & $\lambda_{i}\left(\lambda_{i} \nabla \lambda_{j}-\lambda_{j} \nabla \lambda_{i}\right)$ & $\lambda_{i} \lambda_{j} \nabla \lambda_{k}-\lambda_{j} \lambda_{k} \nabla \lambda_{i}$ & \\
& $\lambda_{j}\left(\lambda_{i} \nabla \lambda_{j}-\lambda_{j} \nabla \lambda_{i}\right)$ & $\lambda_{j} \lambda_{k} \nabla \lambda_{i}-\lambda_{k} \lambda_{i} \nabla \lambda_{j}$ & \\
\hline 3 & $\lambda_{i}^{2}\left(\lambda_{i} \nabla \lambda_{j}-\lambda_{j} \nabla \lambda_{i}\right)$ & $\lambda_{i}\left(\lambda_{i} \lambda_{j} \nabla \lambda_{k}-\lambda_{j} \lambda_{k} \nabla \lambda_{i}\right)$ & $\lambda_{1} \lambda_{2} \lambda_{3} \nabla \lambda_{4}-\lambda_{2} \lambda_{3} \lambda_{4} \nabla \lambda_{1}$ \\
& $\lambda_{i} \lambda_{j}\left(\lambda_{i} \nabla \lambda_{j}-\lambda_{j} \nabla \lambda_{i}\right)$ & $\lambda_{j}\left(\lambda_{i} \lambda_{j} \nabla \lambda_{k}-\lambda_{j} \lambda_{k} \nabla \lambda_{i}\right)$ & $\lambda_{2} \lambda_{3} \lambda_{4} \nabla \lambda_{1}-\lambda_{3} \lambda_{4} \lambda_{1} \nabla \lambda_{2}$ \\
& $\lambda_{j}^{2}\left(\lambda_{i} \nabla \lambda_{j}-\lambda_{j} \nabla \lambda_{i}\right)$ & $\lambda_{k}\left(\lambda_{i} \lambda_{j} \nabla \lambda_{k}-\lambda_{j} \lambda_{k} \nabla \lambda_{i}\right)$ & $\lambda_{3} \lambda_{4} \lambda_{1} \nabla \lambda_{2}-\lambda_{4} \lambda_{1} \lambda_{2} \nabla \lambda_{3}$ \\
& & $\lambda_{i}\left(\lambda_{j} \lambda_{k} \nabla \lambda_{i}-\lambda_{k} \lambda_{i} \nabla \lambda_{j}\right)$ & \\
& & $\lambda_{j}\left(\lambda_{j} \lambda_{k} \nabla \lambda_{i}-\lambda_{k} \lambda_{i} \nabla \lambda_{j}\right)$ & \\
& & $\lambda_{k}\left(\lambda_{j} \lambda_{k} \nabla \lambda_{i}-\lambda_{k} \lambda_{i} \nabla \lambda_{j}\right)$ & \\
& &
\end{tabular}

Table 3.2: Bases given in [17] (up to sign) for $V_{p}(T)$ in terms of barycentric coordinates, $p \leqslant 3$.

As suggested by the discussion of degrees of freedom above, the dimensions of this space is

$$
\operatorname{dim} V_{p}=p \# \mathcal{E}+p(p-1) \# \mathcal{F}+0.5 p(p-1)(p-2) \# \mathcal{T}
$$

For a piecewise polynomial to be in $H(\mathrm{curl})$, it need only have a continuous tangential component across all interior faces in the mesh. It is natural to again split the space $V_{p}$ as $V_{p}=V_{p}^{E} \oplus V_{p}^{F} \oplus V_{p}^{T}$, and consider bases for each part. For each $T \in \mathcal{T}$ we choose our global volume basis functions associated with $T$ to coincide with the local volume basis functions on $T$, extending the local functions by $\mathbf{0}$ outside $T$. Similarly, for each face $F \in \mathcal{F}_{I}$ we choose our global face basis functions associated with $F$ so that they coincide with the local face basis functions on the pair of tetrahedra sharing $F$, and vanish outside of this pair. Finally, for each edge $e \in \mathcal{E}_{I}$ we choose our global edge basis functions so that they coincide with local edge basis functions given above on the ring of tetrahedra sharing $e$, and vanish outside this ring. In this study, we shall always consider the lowest-order $(p=1)$ Nédélec space $V_{p}$ for approximating the 
solution of (2.12), and we define $V=V_{1}$.

\subsection{The discrete variational problem}

The infinite-dimensional space $\mathcal{H}$ is now replaced by a sequence of finite-dimensional spaces $V$, which yields the discrete variational problem of 2.12 : Find $\hat{\mathbf{u}} \in V$ such that

$$
B(\hat{\mathbf{u}}, \mathbf{v})=F(\mathbf{v}) \text { for all } \mathbf{v} \in V
$$

The continuity on $V$ is inherited from the variational problem on $\mathcal{H}$. The generalized Lax-Milgram theorem 2.8 can be applied to the discrete formulation so that the two conditions:

1. There is a constant $\hat{\alpha}>0$ such that

$$
\inf _{\substack{\hat{\mathbf{u}} \in V \\ \hat{\mathbf{u}} \neq 0}} \sup _{\substack{\mathbf{v} \in V \\ \mathbf{v} \neq 0}} \frac{|B(\hat{\mathbf{u}}, \mathbf{v})|}{\|\hat{\mathbf{u}}\|_{V}\|\mathbf{v}\|_{V}} \geqslant \hat{\alpha}
$$

2. For every $\mathbf{v} \in V, \mathbf{v} \neq 0$

$$
\sup _{\hat{\mathbf{u}} \in V}|B(\hat{\mathbf{u}}, \mathbf{v})|>0
$$

imply existence of a unique solution. The former condition 3.14 is called discrete inf-sup condition. A fundamental property, which is called the Galerkin orthogonality, can be obtained by subtracting (3.13) form 2.12, so that

$$
B(\mathbf{u}-\hat{\mathbf{u}}, \mathbf{v})=B(\mathbf{u}, \mathbf{v})-B(\hat{\mathbf{u}}, \mathbf{v})=F(\mathbf{v})-F(\mathbf{v})=0, \text { for all } \mathbf{v} \in V
$$

Lemma 3.5 (Céa). Suppose $V$ is a finite-dimensional subspace of $\mathcal{H}$. Suppose $B(\cdot, \cdot)$ is continuous with bound $C$, and $B(\cdot, \cdot)$ fulfill the discrete inf-sup condition (3.14). Let $\hat{\mathbf{u}} \in V$ solve the discrete problem (3.13). Then, the following error estimate holds:

$$
\|\mathbf{u}-\hat{\mathbf{u}}\|_{H(\operatorname{curl} ; \Omega)} \leqslant\left(1+\frac{C}{\hat{\alpha}}\right)\|\mathbf{u}-\mathbf{v}\|_{H(\operatorname{curl} ; \Omega)}
$$


Proof. Galerkin orthogonality implies $B(\mathbf{u}, \mathbf{w})=B(\hat{\mathbf{u}}, \mathbf{w})$ for all $\mathbf{w} \in V$. For any $\mathbf{v} \in V$ it holds

$$
\|\mathbf{u}-\hat{\mathbf{u}}\|_{H(\operatorname{curl} ; \Omega)} \leqslant\|\mathbf{u}-\mathbf{v}\|_{H(\operatorname{curl} ; \Omega)}+\|\mathbf{v}-\hat{\mathbf{u}}\|_{H(\operatorname{curl} ; \Omega)}
$$

Combining the discrete inf-sup condition (3.14), the Galerkin orthogonality and the continuity of $B$ yields

$$
\begin{aligned}
\|\mathbf{u}-\hat{\mathbf{u}}\|_{H(\operatorname{curl} ; \Omega)} & \leqslant\|\mathbf{u}-\mathbf{v}\|_{H(\operatorname{curl} ; \Omega)}+\hat{\alpha}^{-1} \sup _{\substack{\mathbf{w} \in V \\
\mathbf{w} \neq 0}} \frac{B(\mathbf{v}-\hat{\mathbf{u}}, \mathbf{w})}{\|\mathbf{w}\|_{H(\operatorname{curl} ; \Omega)}} \\
& =\|\mathbf{u}-\mathbf{v}\|_{H(\operatorname{curl} ; \Omega)}+\hat{\alpha}^{-1} \sup _{\substack{\mathbf{w} \in V \\
\mathbf{w} \neq 0}} \frac{B(\mathbf{v}-\mathbf{u}, \mathbf{w})}{\|\mathbf{w}\|_{H(\operatorname{curl} ; \Omega)}} \\
& \leqslant\|\mathbf{u}-\mathbf{v}\|_{H(\operatorname{curl} ; \Omega)}+\hat{\alpha}^{-1} \sup _{\substack{\mathbf{w} \in V \\
\mathbf{w} \neq 0}} \frac{C\|\mathbf{v}-\mathbf{u}\|_{H(\operatorname{curl} ; \Omega)}\|\mathbf{w}\|_{H(\operatorname{curl} ; \Omega)}}{\|\mathbf{w}\|_{H(\operatorname{curl} ; \Omega)}} \\
& =\left(1+\frac{C}{\hat{\alpha}}\right) \mid \mathbf{u}-\mathbf{v} \|_{H(\operatorname{curl} ; \Omega)} .
\end{aligned}
$$

\subsection{The interpolant for $V$}

Associated with the finite element $T, \mathcal{R}_{p}, \Sigma_{T}$ is an interpolant. For a suitable smooth function $\mathbf{u} \in \mathcal{H}$, we define the interpolant on $T$, where $T \in \mathcal{T}$, to be the unique function $\mathbf{r}_{\mathbf{T}} \mathbf{u} \in \mathcal{R}_{p}$ such that

$$
M_{e}\left(\mathbf{u}-\mathbf{r}_{\mathbf{T}} \mathbf{u}\right)=\int_{e}\left(\mathbf{u}-\mathbf{r}_{\mathbf{T}} \mathbf{u}\right) \cdot \mathbf{t} q d s=0 \text { for all } q \in \mathbb{P}_{0} \text { on } e \text { of } T .
$$

The operator $\mathbf{r}_{\mathbf{T}} \mathbf{u}: \mathcal{H} \rightarrow \mathcal{R}_{p}$ is referred to as the interpolation operator. The global interpolant $\mathbf{r}_{\mathbf{h}} \mathbf{u} \in V$ is then defined element by element using $\left.\mathbf{r}_{\mathbf{h}} \mathbf{u}\right|_{T}=\mathbf{r}_{\mathbf{T}} \mathbf{u}$ for all $T \in \mathcal{T}$. In this study, we shall always denote to $\mathbf{r}_{\mathbf{h}} \mathbf{u}$ by $\hat{\mathbf{u}}$.

With our setting for the finite element method, it would be possible to show Theorem 5.41 in [28], which provides a priori error estimates for the interpolant. 
Here we only consider a special case of this theorem, for the general one and for the proof see 28 .

Theorem 3.6. Let $\mathcal{T}$ be a regular mesh on $\Omega$. If $\mathbf{u} \in\left[H^{1}(\Omega)\right]^{3}$ and $\nabla \times \mathbf{u} \in\left[H^{1}(\Omega)\right]^{3}$, then

$$
\|\mathbf{u}-\hat{\mathbf{u}}\|_{H(\text { curl } ; \Omega)}^{2} \leqslant C h\left(\|\mathbf{u}\|_{\left[H^{1}(\Omega)\right]^{3}}^{2}+\|\nabla \times \mathbf{u}\|_{\left[H^{1}(\Omega)\right]^{3}}^{2}\right)
$$

where $C$ is a constant depending on the geometry of the domain and the mesh. 


\section{Chapter 4}

\section{A posteriori Error Estimation}

Having used the Nédélec (edge) element discretization to compute an approximation of the electric field (for example), it is natural to ask how well it approximates the true solution. One often quantifies this discretization error in terms of global norm. A priori error estimates describe the qualitative behavior of the error with respect to parameters governing the discretization, such as the characteristic edge length in a tetrahedral mesh. Although such qualitative estimates provide useful information, in practice one often wants more quantitative estimates of error, both to determine whether or not a discrete solution is "good enough", and to indicate how to intelligently improve the discrete solution if it is not. This is the realm of a posteriori error estimation, which is the focus of our research.

For standard finite element discretizations of PDEs modeling diffusion processes, a posteriori error theory is a mature field, and there are many well-tested techniques on the market. In contrast, a posteriori error theory for Maxwell's equations (by Nédélec elements), is much more recent, and there are relatively few techniques in use that are both theoretically and empirically supported $[5,7,8,11,19,21,22,25,30,34,36$.

\subsection{Hierarchical Based Error Estimator}

Our work will provide provably efficient and reliable error estimates of discretization error based on auxiliary subspace techniques for Nédélec (edge) element discretization of Maxwell's equations on tetrahedral meshes. Such methods have a long history in the context of standard discretizations of diffusion problems, where they are often 
called hierarchical basis error estimators [1, 3, 13, 14, 37]. Such estimators are based on the computation of an approximate error function $\varepsilon \approx \mathbf{u}-\hat{\mathbf{u}}(\hat{\mathbf{u}} \in V$ is a given finite element approximation of the solution $\mathbf{u}$ ) in an auxiliary discrete space $W$ satisfying $V \cap W=\{\mathbf{0}\}$. The traditional analysis of hierarchical error estimators [2,4], in case the bilinear form $B$ is an inner-product with induced "energy norm" $\|\mathbf{v}\|^{2}=B(\mathbf{v}, \mathbf{v})$, makes use of a strong Cauchy inequality between the spaces $V$ and $W$

$$
|B(\mathbf{v}, \mathbf{w})| \leqslant \gamma\|\mathbf{v}\|\|\mathbf{w}\| \text { for all } \mathbf{v} \in V \text { and } \mathbf{w} \in W, \text { where } \gamma \in(0,1),
$$

and a saturation assumption

$$
\|\mathbf{u}-\overline{\mathbf{u}}\| \leqslant \alpha\|\mathbf{u}-\hat{\mathbf{u}}\| \text { for all } \mathbf{u} \in \mathcal{H}, \overline{\mathbf{u}} \in V \oplus W \text {, and } \hat{\mathbf{u}} \in V \text {, where } \alpha \in(0,1),
$$

to obtain upper and lower bounds on error norm $\|\mathbf{u}-\hat{\mathbf{u}}\|$, in terms of the error estimate norm as follows $\|\varepsilon\|$

$$
\|\varepsilon\| \leqslant\|\mathbf{u}-\hat{\mathbf{u}}\| \leqslant \frac{\|\varepsilon\|}{\sqrt{\left(1-\gamma^{2}\right)\left(1-\alpha^{2}\right)}} .
$$

Roughly speaking, the saturation assumption asserts that $\mathbf{u}$ is (strictly) better approximated in the "enriched space" $V \oplus W$ than it is in $V$, and this perspective, though intuitive, often causes viable auxiliary spaces $W$ to be overlooked [18, 20].

Although the saturation assumption is expected to hold in many cases, constructing counter-examples it is not difficult for particular problems on particular meshes. Furthermore, this kind of analysis cannot be readily applied for the more general bilinear forms such as those (that are not inner-products) considered here; and, as suggested above, may unnecessarily limit the types of spaces $W$ in which the error might be approximated. The "saturation assumption approach" was taken in [5] to derive a hierarchical basis error estimator for Maxwell's equations, though it appears that their work has not been pursued further. In this study we will take a different 
approach, to obtain error bounds under weaker (and verifiable) assumptions, using different spaces than in [5]. The principle of this approach is to replace the saturation assumption by "residual oscillation", which appears explicitly in the reliability bound of the error as a computable quantity.

\subsection{Key Error Estimation Result}

Given a finite dimensional supspace $W$ of $\mathcal{H}$ such that $V \cap W=\{\mathbf{0}\}$, we consider the error problem: Find $\varepsilon \in W$ such that

$$
B(\varepsilon, \mathbf{v})=\underbrace{F(\mathbf{v})-B(\hat{\mathbf{u}}, \mathbf{v})}_{B(\mathbf{u}-\hat{\mathbf{u}}, \mathbf{v})} \text { for all } \mathbf{v} \in W .
$$

If $B$ is an inner-product, then $\varepsilon$ is the corresponding orthogonal projection of the error $\mathbf{u}-\hat{\mathbf{u}}$ onto $W$. Even when $B$ is not an inner-product, we may refer to $\varepsilon$ as the projection of $\mathbf{u}-\hat{\mathbf{u}}$ onto $W$. Appropriate global and local norms of $\varepsilon$, e.g. $\|\varepsilon\|_{H(\operatorname{curl} ; \Omega)}$ and $\|\varepsilon\|_{H(\operatorname{curl} ; T)}$, will be used to determine whether the discretization error is small enough; and, if it is not, how it can be efficiently reduced. Clearly, the choice of auxiliary (error) space $W$ is crucial to the success of this approach, and we describe below the motivations for our choice, and the type of theoretical results that we will establish. As mentioned in the introduction, we must balance two objectives in our choice of $W$ :

1. The estimate $\|\varepsilon\|_{H(\operatorname{curl} ; \Omega)}$ of $\|\mathbf{u}-\hat{\mathbf{u}}\|_{H(\operatorname{curl} ; \Omega)}$ should be provably reliable and efficient.

2. The cost of computing $\varepsilon \in W$ should be reasonable, i.e., not more than, and preferably much less than, that of computing $\hat{\mathbf{u}} \in V$.

Having established (1) and (2), we will extend the approach to treat functional measures of error (in chapter 5). 
The starting point of our analysis is the error identity in Proposition 4.1), which follows directly from (2.12), 3.13), and 4.1). The oscillation term in our error bound will come from $B(\mathbf{u}-\hat{\mathbf{u}}, \mathbf{v}-\hat{\mathbf{v}}-\hat{\mathbf{w}})$, so we investigate this term for guidance in choosing $W$. Let $\mathcal{F}$ denote the faces of $\mathcal{T}$ and $\mathbf{g}=\mathbf{v}-\hat{\mathbf{v}}-\hat{\mathbf{w}}$. Writing $B(\mathbf{u}-\hat{\mathbf{u}}, \mathbf{g})=$ $F(\mathbf{g})-B(\hat{\mathbf{u}}, \mathbf{g})$ as a sum of integrals over tetrahedra, and using integration-by-parts on each of these integrals, we obtain,

$$
\begin{aligned}
B(\mathbf{u}-\hat{\mathbf{u}}, \mathbf{g}) & =\int_{\Omega}(\mathbf{f}+\beta \hat{\mathbf{u}}) \cdot \overline{\mathbf{g}}-\left(\mu^{-1} \nabla \times \hat{\mathbf{u}}\right) \cdot \nabla \times \overline{\mathbf{g}} d V \\
& =\sum_{T \in \mathcal{T}}\left(\int_{T}\left(\mathbf{f}+\beta \hat{\mathbf{u}}-\nabla \times\left(\mu^{-1} \nabla \times \hat{\mathbf{u}}\right)\right) \cdot \overline{\mathbf{g}} d V\right. \\
& \left.+\int_{\partial T}\left(\mu^{-1} \nabla \times \hat{\mathbf{u}}\right) \cdot(\overline{\mathbf{g}} \times \mathbf{n}) d A\right)
\end{aligned}
$$

Proposition 4.1. for any $\mathbf{v} \in \mathcal{H}, \hat{\mathbf{v}} \in V$, and $\hat{\mathbf{w}} \in W$, it holds that

$$
B(\mathbf{u}-\hat{\mathbf{u}}, \mathbf{v})=B(\varepsilon, \hat{\mathbf{w}})+B(\mathbf{u}-\hat{\mathbf{u}}, \mathbf{v}-\hat{\mathbf{v}}-\hat{\mathbf{w}})
$$

where

$$
B(\mathbf{u}-\hat{\mathbf{u}}, \mathbf{g})=F(\mathbf{g})-B(\hat{\mathbf{u}}, \mathbf{g})=\sum_{T \in \mathcal{T}} \int_{T} \mathbf{R}_{T} \cdot \overline{\mathbf{g}} d V+\sum_{F \in \mathcal{F}} \int_{F} \mathbf{r}_{F} \cdot(\overline{\mathbf{g}} \times \mathbf{n}) d A
$$

and $\mathbf{R}_{T}=\mathbf{f}+\beta \hat{\mathbf{u}}-\nabla \times\left(\mu^{-1} \nabla \times \hat{\mathbf{u}}\right)$ is the volumetric (strong) residual, and $\mathbf{r}_{F}=$ $\left[\mu^{-1} \nabla \times \hat{\mathbf{u}}\right]_{J}$ is the jump in the weighted curl $\mu^{-1} \nabla \times \hat{\mathbf{u}}$ across the face $F$ once a single normal $\mathbf{n}$ is chosen.

Remark 4.2. If $\mu^{-1}$ is piecewise constant then $\mathbf{r}_{F} \cdot(\overline{\mathbf{g}} \times \mathbf{n})$ will vanish when we make a proper choice of $\mathbf{g}$, and also $\nabla \times\left(\mu^{-1} \nabla \times \hat{\mathbf{u}}\right)=0$ in $\mathbf{R}_{T}$.

With an appropriate choice of error space $W$ (described in detail later), we obtain our key error theorem, 
Theorem 4.3. When $h$ is sufficiently small, then there are constants $k_{1}$ and $k_{2}$ depending on the shape-regularity of $\mathcal{T}$ and problem data $\mu$ and $\beta$ such that

$$
k_{1}\|\varepsilon\|_{H(\operatorname{curl} ; \Omega)} \leqslant\|\mathbf{u}-\hat{\mathbf{u}}\|_{H(\operatorname{curl} ; \Omega)} \leqslant k_{2}\left(\|\varepsilon\|_{H(\operatorname{curl} ; \Omega)}+\operatorname{osc}(R, r, \mathcal{T})\right)
$$

where the residual oscillation is defined by

$$
\operatorname{Osc}(R, r, \mathcal{T})^{2}=\sum_{T \in \mathcal{T}} h_{T}^{2} \inf _{\lambda_{\mathbf{T}} \in \mathbb{R}^{\mathbf{3}}}\left\|\mathbf{R}_{T}-\lambda_{T}\right\|_{L_{2}(T)}^{2}+\sum_{F \in \mathcal{F}} h_{F} \inf _{\kappa_{\mathbf{F}} \in \mathbb{R}^{3}}\left\|\left(\mathbf{r}_{F}-\kappa_{F}\right) \times \mathbf{n}\right\|_{L_{2}(F)}^{2}
$$

Some justification of why might hope to prove such bounds is given in [18, 20, where bounds of this type are proven for a different class of PDE.

The computation of $\varepsilon$ requires the solution of a global system involving the stiffness matrix associated with $W$. At first glance this would seem to rule out the approach as too expensive for practical computations, but we argue herein that this is not the case. In Sect. 4.5 we argue that the matrix associated with the computation of $\varepsilon$, though larger than that associated with the computation of $\hat{\mathbf{u}}$, is much better conditioned. More particularly, we will argue that this matrix is spectrally equivalent to its diagonal, which is certainly not the case for the matrix associated with computing $\hat{\mathbf{u}}$ on $V$. Our key result in this regard is

Theorem 4.4. The global stiffness matrix for $W$ is spectrally-equivalent to its diagonal.

\subsection{Local and Global Error Space $W$}

Choosing the error space $W$ plays important role in our error estimation analysis. In view of the structure of the term $B(\mathbf{u}-\hat{\mathbf{u}}, \mathbf{v}-\hat{\mathbf{v}}-\hat{\mathbf{w}})$ in Proposition 4.1, it is natural to consider an error space $W$ that has degrees of freedom associated with each 
tetrahedron and all (interior) faces in the mesh. We define such a space below, and show some of its key properties that will be instrumental in the proofs of Theorems 4.3 and 4.4 .

The corresponding global finite error space $W$ and its dimensions are given by

$$
\begin{aligned}
W & =\left\{\mathbf{v} \in \mathcal{H}:\left.\mathbf{v}\right|_{T} \in V_{2}^{F}(T) \oplus V_{3}^{T}(T) \text { for all } T \in \mathcal{T}\right\}, \\
\operatorname{dim} W & =2 \# \mathcal{F}+3 \# \mathcal{T} .
\end{aligned}
$$

We decompose $W(T)$ as $W(T)=V_{2}^{F}(T) \oplus V_{3}^{T}(T)$, where all functions in $V_{3}^{T}(T)$ have vanishing face and edge moments, and $V_{2}^{F}(T)$ consists of those functions which have vanishing edge moments. We refer to $V_{2}^{F}(T)$ as the face space, and $V_{3}^{T}(T)$ as the volume space. This decomposition suggests that a natural basis for $W(T)$ would also be decomposed in this manner. The bases of $W(T)$ are given in the Table 3.2 . So, any $\hat{\mathbf{w}} \in W(T)$ is uniquely decomposed as $\hat{\mathbf{w}}=\mathbf{w}_{2}+\mathbf{w}_{3}$ with $\mathbf{w}_{2} \in V_{2}^{F}(T)$ and $\mathbf{w}_{3} \in V_{3}^{T}(T)$.

Remark 4.5. The auxiliary error space $\widetilde{W}$ that used in $[5]$ is defined as

$$
\begin{aligned}
\widetilde{W} & =\left\{\mathbf{v} \in \mathcal{H}:\left.\mathbf{v}\right|_{T} \in \widetilde{V}_{2}^{E}(T) \oplus V_{2}^{F}(T) \text { for all } T \in \mathcal{T}\right\}, \\
\operatorname{dim} \widetilde{W} & =\# \mathcal{E}+2 \# \mathcal{F},
\end{aligned}
$$

where $\tilde{V}_{2}^{E}(T)=V_{2}^{E}(T) \ominus V_{1}^{E}(T)$. In the linear system associated with our error space $W$, we can locally eliminate degrees of freedom associated with each $V_{3}^{T}(T)$, so our global system really only needs to be as large as the twice the number of faces. In the approach used in [5], such a reduction is not possible, so their systems associated with $\widetilde{W}$ are inherently larger and have a more complicated structure.

Lemma 4.6. If $\mathbf{w} \in V_{2}^{F}(T) \oplus V_{3}^{T}(T)$ satisfies $\nabla \times \mathbf{w}=\mathbf{0}$, then $\mathbf{w}=\mathbf{0}$. 
Proof. Using Stoke's Theorem and the Divergence Theorem, we see that for sufficiently smooth $\mathbf{w}, g$ and $\mathbf{g}$,

$$
\begin{aligned}
\oint_{\partial F_{\ell}} \mathbf{w} \cdot \mathbf{t} g d s & =\int_{F_{\ell}} \nabla \times(g \mathbf{w}) \cdot \mathbf{n}_{\ell} d A=\int_{F_{\ell}} g\left(\nabla \times \mathbf{w} \cdot \mathbf{n}_{\ell}\right)+\left(\mathbf{w} \times \mathbf{n}_{\ell}\right) \cdot \nabla g d A, \\
\int_{\partial T}(\mathbf{w} \times \mathbf{n}) \cdot \mathbf{g} d A & =\int_{T} \nabla \cdot(\mathbf{g} \times \mathbf{w}) d V=\int_{T} \mathbf{w} \cdot(\nabla \times \mathbf{g})-\mathbf{g} \cdot(\nabla \times \mathbf{w}) d V,
\end{aligned}
$$

where $\mathbf{t}$ is the unit tangent vector around $\partial F_{\ell}$ and $\mathbf{n}_{\ell}$ is the outward unit normal to face $F_{\ell}$. From this we see that, if $\nabla \times \mathbf{w}=\mathbf{0}$, then

$$
\oint_{\partial F_{\ell}} \mathbf{w} \cdot \mathbf{t} g d s=\int_{F_{\ell}}\left(\mathbf{w} \times \mathbf{n}_{\ell}\right) \cdot \nabla g d A \quad, \quad \int_{\partial T}(\mathbf{w} \times \mathbf{n}) \cdot \mathbf{g} d A=\int_{T} \mathbf{w} \cdot(\nabla \times \mathbf{g}) d V .
$$

Consider $\mathbf{w} \in V_{2}^{F}(T)$ and $\mathbf{w} \in V_{3}^{T}(T)$ separately. For $\mathbf{w} \in V_{p}^{F}(T)$, the edge moments vanish, so we have,

$$
\int_{F_{\ell}}\left(\mathbf{w} \times \mathbf{n}_{\ell}\right) \cdot \nabla g d A=0
$$

for $g \in \mathbb{P}_{p-1}$. When $p=2$, we obtain a full set of face degrees of freedom by choosing $g=\mathbf{c} \cdot x$ for arbitrary $\mathbf{c} \in \mathbb{R}^{3}$. Since all degrees of freedom vanish for $\mathbf{w}$, we deduce that $\mathbf{w}=\mathbf{0}$ when $p=2$.

For $\mathbf{w} \in V_{p}^{T}(T)$ the edge and face moments vanish, so we have

$$
\int_{T} \mathbf{w} \cdot(\nabla \times \mathbf{g}) d V=0
$$

for $\mathbf{g} \in\left[\mathbb{P}_{p-2}\right]^{3}$. When $p=3$ we obtain a full set of volume degrees of freedom by choosing $\mathbf{g}=(b z, c x, a y)$ for arbitrary $(a, b, c) \in \mathbb{R}^{3}$. Since all degrees of freedom vanish for $\mathbf{w}$, we deduce that $\mathbf{w}=\mathbf{0}$ when $p=3$.

In this study, we will use $W_{2}(T)=V_{2}^{F}(T)$ and $W_{3}(T)=V_{3}^{T}(T)$.

Proposition 4.7. For any $T \in \mathcal{T}$ and any $\mathbf{w} \in W(T),\|\nabla \times \mathbf{w}\|_{L^{2}(T)}$ is a norm on $W(T)$. Moreover, there are a scale-invariant constants $c, C>0$ such that

$$
c h_{T}^{-1}\|\mathbf{w}\|_{L^{2}(T)} \leqslant\|\nabla \times \mathbf{w}\|_{L^{2}(T)} \leqslant C h_{T}^{-1}\|\mathbf{w}\|_{L^{2}(T)} \quad \text { for all } \mathbf{w} \in W(T) .
$$


Proof. For any $\mathbf{v}, \mathbf{w} \in W(T)$, let $\langle\mathbf{v}, \mathbf{w}\rangle$ defined by

$$
\langle\mathbf{v}, \mathbf{w}\rangle=\int_{T}(\nabla \times \mathbf{v}) \cdot(\nabla \times \overline{\mathbf{w}}) d V
$$

From this definition and from Lemma 4.6 , we have $\langle\mathbf{v}, \mathbf{v}\rangle=0$ if and only if $\mathbf{v}=0$ and $\langle\alpha \mathbf{v}, \alpha \mathbf{v}\rangle=|\alpha|^{2}\langle\mathbf{v}, \mathbf{v}\rangle$ for any $\alpha \in \mathbb{C}$. In other words, $\|\nabla \times \mathbf{v}\|_{L^{2}(T)}=0 \Leftrightarrow \mathbf{v}=0$ and $\|\nabla \times(\alpha \mathbf{v})\|_{L^{2}(T)}=|\alpha|\|\nabla \times \mathbf{v}\|_{L^{2}(T)}$. Considering $\mathbf{v}, \mathbf{w} \in W(T)$, we have

$$
\begin{aligned}
\|\nabla \times(\mathbf{v}+\mathbf{w})\|_{L^{2}(T)}^{2} & =\langle\mathbf{v}+\mathbf{w}, \mathbf{v}+\mathbf{w}\rangle=\langle\mathbf{v}, \mathbf{v}\rangle+\langle\mathbf{w}, \mathbf{w}\rangle+\langle\mathbf{v}, \mathbf{w}\rangle+\langle\mathbf{w}, \mathbf{v}\rangle \\
& =\|\nabla \times \mathbf{v}\|_{L^{2}(T)}^{2}+\|\nabla \times \mathbf{w}\|_{L^{2}(T)}^{2}+2 \Re(\langle\mathbf{v}, \mathbf{w}\rangle)
\end{aligned}
$$

and using Cauchy-Schwarz inequality,

$$
\begin{aligned}
\|\nabla \times(\mathbf{v}+\mathbf{w})\|_{L^{2}(T)}^{2} & \leqslant\|\nabla \times \mathbf{v}\|_{L^{2}(T)}^{2}+\|\nabla \times \mathbf{w}\|_{L^{2}(T)}^{2}+2|\langle\mathbf{v}, \mathbf{w}\rangle| \\
& \leqslant\|\nabla \times \mathbf{v}\|_{L^{2}(T)}^{2}+\|\nabla \times \mathbf{w}\|_{L^{2}(T)}^{2}+2 \sqrt{\langle\mathbf{v}, \mathbf{v}\rangle\langle\mathbf{w}, \mathbf{w}\rangle} \\
& \leqslant\|\nabla \times \mathbf{v}\|_{L^{2}(T)}^{2}+\|\nabla \times \mathbf{w}\|_{L^{2}(T)}^{2}+2\|\nabla \times \mathbf{v}\|_{L^{2}(T)}\|\nabla \times \mathbf{w}\|_{L^{2}(T)} \\
& =\left(\|\nabla \times \mathbf{v}\|_{L^{2}(T)}+\|\nabla \times \mathbf{w}\|_{L^{2}(T)}\right)^{2}
\end{aligned}
$$

Hence, $\|\nabla \times(\mathbf{v}+\mathbf{w})\|_{L^{2}(T)} \leqslant\|\nabla \times \mathbf{v}\|_{L^{2}(T)}+\|\nabla \times \mathbf{w}\|_{L^{2}(T)}$ (triangle inequality). Therefore $\|\nabla \times \mathbf{v}\|_{L^{2}(T)}$ is a norm on $W(T)$.

Let $\tilde{T}=\left\{\tilde{x}=h_{T}^{-1} x: x \in T\right\}$ where $T$ is a physical element with $\operatorname{diam}(T)=h_{T}$ and $\tilde{T}$ is a reference element with $\operatorname{diam}(\tilde{T})=1$. For each $\mathbf{w}: W(T) \rightarrow \mathbb{R}$, define $\tilde{\mathbf{w}}: W(\tilde{T}) \rightarrow \mathbb{R}$ by $\tilde{\mathbf{w}}(\tilde{x})=\mathbf{w}\left(h_{T} \tilde{x}\right)=\mathbf{w}(x)$. It is clear that

$$
\begin{aligned}
\|\mathbf{w}\|_{L^{2}(T)}^{2} & =\int_{T}|\mathbf{w}(x)|^{2} d V=\int_{\tilde{T}}|\tilde{\mathbf{w}}(\tilde{x})|^{2} h_{T}^{3} d \tilde{x}=h_{T}^{3}\|\tilde{\mathbf{w}}\|_{0, \tilde{T}}^{2}, \\
\|\nabla \times \mathbf{w}\|_{L^{2}(T)}^{2} & =\int_{T}|\nabla \times \mathbf{w}(x)|^{2} d V=\int_{\tilde{T}} h_{T}^{-2}|\nabla \times \tilde{\mathbf{w}}(\tilde{x})|^{2} h_{T}^{3} d \tilde{x}=h_{T}\|\nabla \times \tilde{\mathbf{w}}\|_{0, \tilde{T}}^{2} .
\end{aligned}
$$


$W(\tilde{T})$ is a finite dimensional vector space on $\tilde{T} .\|\cdot\|_{0, \tilde{T}}$ and $\|\nabla \times \cdot\|_{0, \tilde{T}}$ are both norms on $W(\tilde{T})$, so there are constants $c, C$ such that

$$
c\|\tilde{\mathbf{w}}\|_{0, \tilde{T}} \leqslant\|\nabla \times \tilde{\mathbf{w}}\|_{0, \tilde{T}} \leqslant C\|\tilde{\mathbf{w}}\|_{0, \tilde{T}} \quad \text { for all } \tilde{\mathbf{w}} \in W(\tilde{T}) .
$$

From 4.6) and 4.7), we have

$$
c h_{T}^{-1}\|\mathbf{w}\|_{L^{2}(T)} \leqslant\|\nabla \times \mathbf{w}\|_{L^{2}(T)} \leqslant C h_{T}^{-1}\|\mathbf{w}\|_{L^{2}(T)}
$$

Proposition 4.8. Suppose that $\beta$ is real on $\mathcal{T}$ and $\mu$ satisfies $\mu^{-1} \geqslant m>0$ on $\Omega$ for some real $m$. Given $T \in \mathcal{T}$ and $\mathbf{w} \in W(T)$, there is a constant $\sigma_{T} \geqslant m / 2$ for which $B_{T}(\mathbf{w}, \mathbf{w}) \geqslant \sigma_{T}\|\nabla \times \mathbf{w}\|_{L^{2}(T)}^{2}$ when $h_{T}$ is sufficiently small.

Proof. Let $B_{T}(\mathbf{u}, \mathbf{v})$ be the element-wise bilinear form for $B(\mathbf{u}, \mathbf{v})$. Then we have

$$
\begin{aligned}
B_{T}(\mathbf{w}, \mathbf{w}) & =\int_{T} \mu_{T}^{-1}|\nabla \times \mathbf{w}|^{2} d V-\int_{T} \beta_{T}|\mathbf{w}|^{2} d V \\
& \geqslant \mu_{T}^{-1}\|\nabla \times \mathbf{w}\|_{L^{2}(T)}^{2}-\|\beta\|_{L^{\infty}(T)}\|\mathbf{w}\|_{L^{2}(T)}^{2} d V .
\end{aligned}
$$

From 4.7, we obtain

$$
\begin{aligned}
B_{T}(\mathbf{w}, \mathbf{w}) & \geqslant \mu_{T}^{-1}\|\nabla \times \mathbf{w}\|_{L^{2}(T)}^{2}-\|\beta\|_{L^{\infty}(T)} c_{T} h_{T}^{2}\|\nabla \times \mathbf{w}\|_{L^{2}(T)}^{2} \\
& =\left(\mu_{T}^{-1}-\|\beta\|_{L^{\infty}(T)} c_{T} h_{T}^{2}\right)\|\nabla \times \mathbf{w}\|_{L^{2}(T)}^{2} \\
& =\sigma_{T}\|\nabla \times \mathbf{w}\|_{L^{2}(T)}^{2},
\end{aligned}
$$

where $\sigma_{T}=\mu_{T}^{-1}-\|\beta\|_{L^{\infty}(T)} c_{T} h_{T}^{2}$. When $h_{T}$ is small enough, $\sigma_{T} \geqslant \frac{\mu_{T}^{-1}}{2} \geqslant \frac{m}{2}>0$.

\subsection{Reliability Analysis}

Before we prove Theorem 4.3 , we need to establish several theorems and lemmas. We will need the small domains (patches) associated with vertices and elements of the 
mesh. The following definitions of patches will be useful in the following analysis. For a given vertex $z \in \mathcal{V}$, we assign to it the patch $\Omega_{z}=\bigcup\{T: z \in \partial T\}$. Then we define the domains

$$
\tilde{\Omega}_{z}=\bigcup_{z^{\prime} \in \Omega_{z}} \Omega_{z^{\prime}} \quad \text { and } \quad \tilde{\Omega}_{T}=\bigcup_{z^{\prime} \in T} \tilde{\Omega}_{z^{\prime}} .
$$

containing the neighbor elements of neighbor elements of a vertex $z$ and an element $T$, respectively.

Theorem 4.9. [34, Theorem 1]. There exists an operator $\Pi_{E}: H_{0}(\operatorname{curl} ; \Omega) \rightarrow V$ with the following properties: For every $\mathbf{v} \in H_{0}(\operatorname{curl} ; \Omega)$ there exists $\phi \in H_{0}^{1}(\Omega)$ and $\boldsymbol{\psi} \in\left[H_{0}^{1}(\Omega)\right]^{3}$ such that

$$
\mathbf{v}-\Pi_{E} \mathbf{v}=\boldsymbol{\psi}+\nabla \phi
$$

The decomposition satisfies

$$
\begin{aligned}
& h_{T}^{-1}\|\phi\|_{L^{2}(T)}+\|\nabla \phi\|_{L^{2}(T)} \leqslant C\|\mathbf{v}\|_{L^{2}\left(\tilde{\Omega}_{T}\right)}, \\
& h_{T}^{-1}\|\boldsymbol{\psi}\|_{L^{2}(T)}+\|\nabla \boldsymbol{\psi}\|_{L^{2}(T)} \leqslant C\|\nabla \times \mathbf{v}\|_{L^{2}\left(\tilde{\Omega}_{T}\right)} .
\end{aligned}
$$

The constant $C$ depends only on the shape of the elements in the enlarged element patch $\tilde{\Omega}_{T}$, but does not depend on the global shape of the domain $\Omega$ or the size of the patch $\tilde{\Omega}_{T}$.

Lemma 4.10. Let $\mathbf{v}, \phi$ and $\boldsymbol{\psi}$ satisfy the decomposition in Theorem 4.9. Then there are scale-invariant constants $C_{1}$ and $C_{2}$ such that

(i) $\|\phi\|_{L^{2}(F)} \leqslant C_{1} h_{T}^{1 / 2}\|\mathbf{v}\|_{L^{2}\left(\tilde{\Omega}_{T}\right)}$,

(ii) $\|\boldsymbol{\psi}\|_{L^{2}(F)} \leqslant C_{2} h_{T}^{1 / 2}\|\nabla \times \mathbf{v}\|_{L^{2}\left(\tilde{\Omega}_{T}\right)}$

where $T$ is a tetrahedron having $F$ as a face. 
Proof. Let $z_{F}$ be the vertex of $T$ opposite the face $F$. From the Divergence Theorem, we have

$$
\int_{T} \nabla \cdot\left(\phi^{2}\left(x-z_{F}\right)\right) d V=\int_{\partial T} \phi^{2}\left(x-z_{F}\right) \cdot \mathbf{n} d s=\alpha_{F}\|\phi\|_{L^{2}(F)}^{2}
$$

where $\alpha_{F}$ is the distance from $z_{F}$ to the face $F$. Also

$$
\begin{aligned}
\int_{T} \nabla \cdot\left(\phi^{2}\left(x-z_{F}\right)\right) d V & =\int_{T} \nabla \cdot\left(x-z_{F}\right) \phi^{2} d V+\int_{T}\left(x-z_{F}\right) \cdot \nabla\left(\phi^{2}\right) d V \\
& =3\|\phi\|_{L^{2}(T)}^{2}+\int_{T}\left(2 \phi \nabla \phi \cdot\left(x-z_{F}\right) d V\right.
\end{aligned}
$$

Using Young's inequality and 4.8, we have

$$
\begin{aligned}
\alpha_{T}\|\phi\|_{L^{2}(F)}^{2} & \leqslant 3\|\phi\|_{L^{2}(T)}^{2}+\int_{T} \phi^{2}+\left(\nabla \phi \cdot\left(x-z_{F}\right)\right)^{2} d V \\
\|\phi\|_{L^{2}(F)}^{2} & \leqslant \frac{4}{\alpha_{T}}\|\phi\|_{L^{2}(T)}^{2}+\frac{h_{T}^{2}}{\alpha_{T}}\|\nabla \phi\|_{L^{2}(T)}^{2} \\
& \lesssim h_{T}^{-1}\|\phi\|_{L^{2}(T)}^{2}+\tilde{c} h_{T}\|\nabla \phi\|_{L^{2}(T)}^{2} \\
& =h_{T}\left(h_{T}^{-1}\|\phi\|_{L^{2}(T)}\right)^{2}+\tilde{c} h_{T}\|\nabla \phi\|_{L^{2}(T)}^{2}
\end{aligned}
$$

From Theorem 4.9, we have

$$
\|\phi\|_{L^{2}(F)}^{2} \lesssim h_{T}\|\mathbf{v}\|_{L^{2}\left(\tilde{\Omega}_{T}\right)}^{2}+h_{T}\|\mathbf{v}\|_{L^{2}\left(\tilde{\Omega}_{T}\right)}^{2}=2 h_{T}\|\mathbf{v}\|_{L^{2}\left(\tilde{\Omega}_{T}\right)}^{2} .
$$

Taking square roots gives

$$
\|\phi\|_{L^{2}(F)} \leqslant C_{1} h_{T}^{1 / 2}\|\mathbf{v}\|_{L^{2}\left(\tilde{\Omega}_{T}\right)},
$$

where $C_{1}$ is a constant that depends only on the shape of the elements in $\tilde{\Omega}_{T}$.

Now we prove (ii). We note that $\boldsymbol{\psi}$ is a vector, so by repeating same steps above with the components of $\boldsymbol{\psi}$, we obtain

$$
\|\boldsymbol{\psi}\|_{L^{2}(F)}^{2} \lesssim h_{T}\left(h_{T}^{-1}\|\boldsymbol{\psi}\|_{L^{2}(T)}\right)^{2}+h_{T}\|\nabla \boldsymbol{\psi}\|_{L^{2}(T)}^{2}
$$


and from Theorem 4.9, we have

$$
\|\boldsymbol{\psi}\|_{L^{2}(F)}^{2} \lesssim h_{T}\|\nabla \times \mathbf{v}\|_{L^{2}\left(\tilde{\Omega}_{T}\right)}^{2}+h_{T}\|\nabla \times \mathbf{v}\|_{L^{2}\left(\tilde{\Omega}_{T}\right)}^{2}=2 h_{T}\|\nabla \times \mathbf{v}\|_{L^{2}\left(\tilde{\Omega}_{T}\right)}^{2} .
$$

Taking square roots gives

$$
\|\boldsymbol{\psi}\|_{L^{2}(F)} \leqslant C_{2} h_{T}^{1 / 2}\|\nabla \times \mathbf{v}\|_{L^{2}\left(\tilde{\Omega}_{T}\right)},
$$

where $C_{2}$ is a constant that depends only on the shape of the elements in $\tilde{\Omega}_{T}$.

Lemma 4.11. Let $\mathbf{v} \in H_{0}(\operatorname{curl} ; \Omega)$, and let $\boldsymbol{\psi} \in\left[H_{0}^{1}(\Omega)\right]^{3}$ be the function guaranteed in Theorem 4.9. There exists $\mathbf{w}_{2} \in W_{2}(T)$, which satisfies

$$
\int_{F} \mathbf{w}_{2} \cdot(\mathbf{q} \times \mathbf{n}) d s=\int_{F} \boldsymbol{\psi} \cdot(\mathbf{q} \times \mathbf{n}) d s \text { for all } \mathbf{q} \in\left[\mathbb{P}_{0}(F)\right]^{3} \text { and } F \in \mathcal{F} .
$$

For this $\mathbf{w}_{2}$, there are scale-invariant constants $C_{1}$ and $C_{2}$ such that

(i) $\left\|\mathbf{w}_{2}\right\|_{L^{2}(T)} \leqslant C_{1} h_{T}\|\nabla \times \mathbf{v}\|_{L^{2}\left(\tilde{\Omega}_{T}\right)}$ for all $T \in \mathcal{T}$,

(ii) $\left\|\mathbf{w}_{2}\right\|_{L^{2}(F)} \leqslant C_{2} h_{F}^{1 / 2}\|\nabla \times \mathbf{v}\|_{L^{2}\left(\tilde{\Omega}_{T}\right)}$ for all $F \in \mathcal{F}$,

where $T$ is a tetrahedron having $F$ as a face.

Proof. Using Cauchy-Schwarz inequality for 4.9, we obtain

$$
\frac{\left|\int_{F} \mathbf{w}_{2} \cdot(\mathbf{q} \times \mathbf{n}) d s\right|}{\|\mathbf{q} \times \mathbf{n}\|_{L^{2}(F)}} \leqslant\|\boldsymbol{\psi}\|_{L^{2}(F)} .
$$

From Lemma 4.10 , we have $\|\boldsymbol{\psi}\|_{L^{2}(\partial T)} \lesssim h_{T}^{1 / 2}\|\nabla \times \mathbf{v}\|_{L^{2}\left(\tilde{\Omega}_{T}\right)}$. So,

$$
h_{T}^{-1 / 2} \frac{\left|\int_{F} \mathbf{w}_{2} \cdot(\mathbf{q} \times \mathbf{n}) d s\right|}{\|\mathbf{q} \times \mathbf{n}\|_{L^{2}(F)}} \lesssim\|\nabla \times \mathbf{v}\|_{L^{2}\left(\tilde{\Omega}_{T}\right)} .
$$

Since the functions in $W_{2}(T)$ are uniquely determined by 10 , the mapping $\langle\langle\cdot\rangle\rangle$ : $W_{2}(T) \rightarrow \mathbb{R}^{+}$defined by

$$
\langle\langle\omega\rangle\rangle=\max _{F \in \mathcal{F}(T)} \sup _{q \in\left[\mathbb{P}_{0}(F)\right]^{3}} \frac{h_{T}^{-1 / 2}}{\|\mathbf{q} \times \mathbf{n}\|_{L^{2}(F)}} \int_{F} \omega \cdot(\mathbf{q} \times \mathbf{n}) d s
$$


is a norm on $W_{2}(T)$. From Proposition $4.7,\left\|\nabla \times \mathbf{w}_{2}\right\|_{L^{2}(T)}$ is a norm on $W_{2}(T)$, so it is equivalent to $\left\langle\left\langle\mathbf{w}_{2}\right\rangle\right\rangle$. A scaling argument shows that they scale the same way. As consequence of this, we have

$$
\left\|\nabla \times \mathbf{w}_{2}\right\|_{L^{2}(T)} \lesssim\|\nabla \times \mathbf{v}\|_{L^{2}\left(\tilde{\Omega}_{T}\right)} .
$$

Also from Proposition 4.7, we have the following norm equivalence on $W_{2}(T)$,

$$
\left\|\mathbf{w}_{2}\right\|_{L^{2}(T)} \lesssim h_{T}\left\|\nabla \times \mathbf{w}_{2}\right\|_{L^{2}(T)}
$$

Therefore

$$
\left\|\mathbf{w}_{2}\right\|_{L^{2}(T)} \leqslant C_{1} h_{T}\|\nabla \times \mathbf{v}\|_{L^{2}\left(\tilde{\Omega}_{T}\right)} .
$$

Now we prove (ii). We claim that $\|\cdot\|_{L^{2}(\partial T)}$ is a norm on $W_{2}(T)$. To see that this is so, suppose that $\mathbf{w}_{2} \in W_{2}(T)$ and $\left\|\mathbf{w}_{2}\right\|_{L^{2}(\partial T)}=0$. Then $\left\|\mathbf{w}_{2}\right\|_{L^{2}(F)}=0$ for each face $F$ of $T$, so $\mathbf{w}_{2}=\mathbf{0}$ on each face $F$ of $T$. This means that

$$
\int_{F} \mathbf{w}_{2} \cdot(\mathbf{q} \times \mathbf{n}) d s=0 \text { for all } \mathbf{q} \in\left[\mathbb{P}_{0}(F)\right]^{3} \text { and } F \in \mathcal{F} .
$$

Then $\mathbf{w}_{2}=\mathbf{0}$ on $T$.

This implies that $\|\cdot\|_{L^{2}(\partial T)}$ is a norm on $W_{2}(T)$. Equivalence of norms on $W_{2}(T)$, and a scaling argument, shows that

$$
\left\|\mathbf{w}_{2}\right\|_{L^{2}(\partial T)} \lesssim h_{T}^{-1 / 2}\left\|\mathbf{w}_{2}\right\|_{L^{2}(T)}
$$

Therefore,

$$
\begin{aligned}
\left\|\mathbf{w}_{2}\right\|_{L^{2}(F)} & \leqslant\left\|\mathbf{w}_{2}\right\|_{L^{2}(\partial T)} \lesssim h_{T}^{-1 / 2}\left\|\mathbf{w}_{2}\right\|_{L^{2}(T)} \\
& \lesssim h_{T}^{1 / 2}\|\nabla \times \mathbf{v}\|_{L^{2}\left(\tilde{\Omega}_{T}\right)}
\end{aligned}
$$

Using the shape regularity of the mesh, we have

$$
\left\|\mathbf{w}_{2}\right\|_{L^{2}(F)} \leqslant C_{2} h_{F}^{1 / 2}\|\nabla \times \mathbf{v}\|_{L^{2}\left(\tilde{\Omega}_{T}\right)}
$$


Lemma 4.12. Let $\mathbf{v} \in H_{0}(\operatorname{curl} ; \Omega)$, and let $\boldsymbol{\psi} \in\left[H_{0}^{1}(\Omega)\right]^{3}$ and $\mathbf{w}_{2} \in W_{2}(\mathcal{T})$ be the functions guaranteed in Theorem 4.9 and Lemma 4.11. There exists $\mathbf{w}_{3} \in W_{3}(T)$, which satisfies

$$
\int_{T} \mathbf{w}_{3} \cdot q d V=\int_{T}\left(\mathbf{w}_{2}-\boldsymbol{\psi}\right) \cdot q d V \text { for all } q \in\left[\mathbb{P}_{0}(T)\right]^{3} \text { and } T \in \mathcal{T} .
$$

For this $\mathbf{w}_{3}$, there is a scale-invariant constant $C$ such that

$$
\left\|\mathbf{w}_{3}\right\|_{L^{2}(T)} \leqslant C h_{T}\|\nabla \times \mathbf{v}\|_{L^{2}\left(\tilde{\Omega}_{T}\right)} \text { for all } T \in \mathcal{T}
$$

Proof. Using Cauchy-Schwarz inequality for 4.10, we obtain

$$
\frac{\left|\int_{T} \mathbf{w}_{3} \cdot q d V\right|}{\|q\|_{L^{2}(T)}} \leqslant\|\boldsymbol{\psi}\|_{L^{2}(T)}+\left\|\mathbf{w}_{2}\right\|_{L^{2}(T)}
$$

from Theorem 4.9 and Lemma 4.11 , we have $\|\boldsymbol{\psi}\|_{L^{2}(T)}+\left\|\mathbf{w}_{2}\right\|_{L^{2}(T)} \lesssim h_{T}\|\nabla \times \mathbf{v}\|_{L^{2}\left(\tilde{\Omega}_{T}\right)}$. So,

$$
h_{T}^{-1} \frac{\left|\int_{T} \mathbf{w}_{3} \cdot q d V\right|}{\|q\|_{L^{2}(T)}} \lesssim\|\nabla \times \mathbf{v}\|_{L^{2}\left(\tilde{\Omega}_{T}\right)} .
$$

Since the functions in $W_{3}(T)$ are uniquely determined by the values (11), the mapping $\langle\langle\cdot\rangle\rangle: W_{3}(T) \rightarrow \mathbb{R}^{+}$defined by

$$
\langle\langle\omega\rangle\rangle=\sup _{q \in\left[\mathbb{P}_{0}(F)\right]^{3}} \frac{h_{T}^{-1 / 2}}{\|q\|_{L^{2}(T)}} \int_{T} \omega \cdot q d V
$$

is a norm on $W_{3}(T)$. From Proposition $4.7,\left\|\nabla \times \mathbf{w}_{3}\right\|_{L^{2}(T)}$ is a norm on $W_{3}(T)$, then it is equivalent to $\left\langle\left\langle\mathbf{w}_{3}\right\rangle\right\rangle$. A scaling argument shows that they scale the same way. As consequence of this, we have

$$
\left\|\nabla \times \mathbf{w}_{3}\right\|_{L^{2}(T)} \lesssim\|\nabla \times \mathbf{v}\|_{L^{2}\left(\tilde{\Omega}_{T}\right)}
$$

Also from Proposition 4.7, we have the following norm equivalence on $W_{3}(T)$,

$$
\left\|\mathbf{w}_{3}\right\|_{L^{2}(T)} \lesssim h_{T}\left\|\nabla \times \mathbf{w}_{3}\right\|_{L^{2}(T)}
$$


Therefore

$$
\left\|\mathbf{w}_{3}\right\|_{L^{2}(T)} \leqslant C h_{T}\|\nabla \times \mathbf{v}\|_{L^{2}\left(\tilde{\Omega}_{T}\right)} .
$$

Let $S(\mathcal{T})=\left\{s \in \mathcal{C}(\bar{\Omega}):\left.s\right|_{T} \in \mathbb{P}_{1}(T)\right.$ for all $\left.T \in \mathcal{T}\right\}$, and $S_{0}(\mathcal{T})=S(\mathcal{T}) \cap H_{0}^{1}(\Omega)$. It follows from 2.10 that $\nabla S(\mathcal{T}) \subset V(\mathcal{T})$. Using this space, we say a function $\mathbf{u} \in\left(L^{2}(\Omega)\right)^{3}$ is discrete divergence-free if

$$
\int_{\Omega} \mathbf{u} \cdot \nabla s d V=0 \text { for all } s \in S_{0}(\mathcal{T})
$$

The space of discrete divergence-free is denoted by $\tilde{V}$.

According to the arguments in [28, Section 7.2], for a given discrete divergence-free function $\tilde{\mathbf{v}} \in \tilde{V}$, there is a $\xi \in H_{0}(\operatorname{curl} ; \Omega)$ satisfying

$$
\nabla \times \xi=\nabla \times \tilde{\mathbf{v}} \text { and } \nabla \cdot \xi=0 \text { in } \Omega
$$

Lemma 4.13. [28, Lemma 7.6] Let $\tilde{\mathbf{v}} \in \tilde{V}$. Suppose $\xi \in H_{0}(\operatorname{curl} ; \Omega)$ satisfies 4.12. Then there are constants $C$ and $\delta>0$ independent of $h$ and $\tilde{\mathbf{v}}$ and $\xi$ such that

$$
\|\xi-\tilde{\mathbf{v}}\|_{L^{2}(\Omega)} \leqslant C h^{1 / 2+\delta}\|\nabla \times \tilde{\mathbf{v}}\|_{L^{2}(\Omega)}
$$

Lemma 4.14. Suppose $\mathbf{f} \in H(\operatorname{curl} ; \Omega)$, and let $\phi \in H_{0}^{1}(\Omega)$ be the function guaranteed in Theorem 4.9. Then there are constants $C$ and $\delta>0$ such that

$$
\left|\int_{\Omega}(\mathbf{f}+\beta \hat{\mathbf{u}}) \cdot \nabla \phi d V\right| \leqslant C|\beta| h^{1 / 2+\delta}\|\nabla \times(\mathbf{u}-\hat{\mathbf{u}})\|_{L^{2}(\Omega)}\|\mathbf{v}\|_{L^{2}(\Omega)} .
$$

Proof. Let $A=\int_{\Omega}(\mathbf{f}+\beta \hat{\mathbf{u}}) \cdot \nabla \phi d x$. We first note that using the test function $\mathbf{v}=\nabla s$, $s \in S_{0}(\Omega)$ in 3.13 shows that $\mathbf{f}+\beta \hat{\mathbf{u}}$ is discrete divergence-free since

$$
\int_{\Omega}(\mathbf{f}+\beta \hat{\mathbf{u}}) \cdot \nabla s d V=0 \text { for all } s \in S_{0}(\Omega) .
$$


Therefore, from Lemma 4.13, we have the estimate

$$
\|(\mathbf{f}+\beta \hat{\mathbf{u}})-\xi\|_{L^{2}(\Omega)} \lesssim h^{1 / 2+\delta}\|\nabla \times(\mathbf{f}+\beta \hat{\mathbf{u}})\|_{L^{2}(\Omega)}
$$

for some $\delta>0$. Since $\int_{\Omega} \xi \cdot \nabla \phi d x=\int_{\partial \Omega} \xi \cdot \mathbf{n} \phi d s-\int_{\Omega} \nabla \cdot \xi \phi d x=0$, we have

$$
|A|=\left|\int_{\Omega}(\mathbf{f}+\beta \hat{\mathbf{u}}-\xi) \cdot \nabla \phi d x\right| \lesssim h^{1 / 2+\delta}\|\nabla \times(\mathbf{f}+\beta \hat{\mathbf{u}})\|_{L^{2}(\Omega)}\|\mathbf{u}\|_{L^{2}(\Omega)} .
$$

We also note that

$$
\int_{\Omega}(\mathbf{f}+\beta \hat{\mathbf{u}}) \cdot \nabla \eta d x=\int_{\Omega} \beta(\hat{\mathbf{u}}-\mathbf{u}) \cdot \nabla \eta d x \text { for all } \eta \in H_{0}^{1}(\Omega) .
$$

Using essentially the same reasoning as above, we have an alternate estimate of $A$,

$$
|A| \leqslant C|\beta| h^{1 / 2+\delta}\|\nabla \times(\mathbf{u}-\hat{\mathbf{u}})\|_{L^{2}(\Omega)}\|v\|_{L^{2}(\Omega)}
$$

Here, we assumed that $\beta$ was constant. This second estimate is not a posteriori in the sense that $\|\nabla \times(\mathbf{u}-\hat{\mathbf{u}})\|_{L^{2}(\Omega)}$ is not computable, but it does indicate that $|A|$ is smaller than $\|\mathbf{u}-\hat{\mathbf{u}}\|_{H(\operatorname{curl} ; \Omega)}$ when $h$ is sufficiently small.

Lemma 4.15. Let $\delta>0$ be the constant guaranteed in Lemma 4.14. Given $\mathbf{v} \in$ $\mathcal{H}, \hat{\mathbf{v}} \in V, \hat{\mathbf{w}} \in W$, and $\mathbf{f} \in H(\operatorname{curl} ; \Omega)$. Then

$$
|B(\mathbf{u}-\hat{\mathbf{u}}, \mathbf{v}-\hat{\mathbf{v}}-\hat{\mathbf{w}})| \lesssim \widetilde{O S C}(\mathbf{u}, \hat{\mathbf{u}}, R, r, \mathcal{T})\|\mathbf{v}\|_{H(\operatorname{curl} ; \Omega)}
$$

where

$$
\begin{aligned}
\widetilde{\mathrm{OSC}}(\mathbf{u}, \hat{\mathbf{u}}, R, r, \mathcal{T}) & =h^{1 / 2+\delta}\|\nabla \times(\mathbf{u}-\hat{\mathbf{u}})\|_{L^{2}(\Omega)}+\sum_{T \in \mathcal{T}} h_{T} \inf _{\lambda_{\mathbf{T}} \in \mathbb{R}^{\mathbf{3}}}\left\|\mathbf{R}_{T}-\lambda_{T}\right\|_{L^{2}(T)} \\
& +\sum_{F \in \mathcal{F}} h_{F}^{1 / 2} \inf _{\kappa_{\mathbf{F}} \in \mathbb{R}^{\mathbf{3}}}\left\|\left(\mathbf{r}_{F}-\kappa_{F}\right) \times \mathbf{n}\right\|_{L^{2}(F)},
\end{aligned}
$$

and $\mathbf{R}_{T}=\mathbf{f}+\beta \hat{\mathbf{u}}-\nabla \times\left(\mu^{-1} \nabla \times \hat{\mathbf{u}}\right)$ is the volumetric (strong) residual, and $\mathbf{r}_{F}=$ $\left[\mu^{-1} \nabla \times \hat{\mathbf{u}}\right]_{J}$ is the jump in the weighted curl $\mu^{-1} \nabla \times \hat{\mathbf{u}}$ across the face $F$ once a single normal $\mathbf{n}$ is chosen. 
Remark 4.16. When $\mu^{-1}$ is piecewise constant, the part of the oscillation associated with faces vanishes entirely, and $\mathbf{R}_{T}$ reduces to $\mathbf{f}+\beta \hat{\mathbf{u}}$.

Proof. From Proposition 4.1, we have

$$
B(\mathbf{u}-\hat{\mathbf{u}}, \mathbf{v})=B(\mathbf{u}-\hat{\mathbf{u}}, \mathbf{v}-\hat{\mathbf{v}}-\hat{\mathbf{w}})+B(\varepsilon, \hat{\mathbf{w}})
$$

where

$$
\begin{aligned}
B(\mathbf{u}-\hat{\mathbf{u}}, \mathbf{v}-\hat{\mathbf{v}}-\hat{\mathbf{w}}) & =\int_{\Omega}(\mathbf{f}+\beta \hat{\mathbf{u}}) \cdot(\mathbf{v}-\hat{\mathbf{v}}-\hat{\mathbf{w}}) d V \\
& -\int_{\Omega}\left(\mu^{-1} \nabla \times \hat{\mathbf{u}}\right) \cdot \nabla \times(\mathbf{v}-\hat{\mathbf{v}}-\hat{\mathbf{w}}) d V
\end{aligned}
$$

Decomposing $\hat{\mathbf{w}} \in W(T)$ as $\hat{\mathbf{w}}=\mathbf{w}_{2}+\mathbf{w}_{3}$ with $\mathbf{w}_{2} \in W_{2}(T)$ and $\mathbf{w}_{3} \in W_{3}(T)$, and using the identity $\mathbf{v}-\hat{\mathbf{v}}=\mathbf{v}-\Pi_{E} \mathbf{v}=\boldsymbol{\psi}+\nabla \phi$ from Theorem 4.9, we rewrite the above as.

$$
\begin{aligned}
B(\mathbf{u}-\hat{\mathbf{u}}, \mathbf{v}-\hat{\mathbf{v}}-\hat{\mathbf{w}}) & =\int_{\Omega}(\mathbf{f}+\beta \hat{\mathbf{u}}) \cdot \nabla \phi d V \\
& +\sum_{T \in \mathcal{T}} \int_{T}(\mathbf{f}+\beta \hat{\mathbf{u}}) \cdot\left(\boldsymbol{\psi}-\mathbf{w}_{2}-\mathbf{w}_{3}\right) d V \\
& -\sum_{T \in \mathcal{T}} \int_{T}\left(\mu^{-1} \nabla \times \hat{\mathbf{u}}\right) \cdot \nabla \times\left(\boldsymbol{\psi}-\mathbf{w}_{2}-\mathbf{w}_{3}\right) d V
\end{aligned}
$$

Using integration-by-parts on the terms in the final sum, we have

$$
\begin{aligned}
B(\mathbf{u}-\hat{\mathbf{u}}, \mathbf{v}-\hat{\mathbf{v}}-\hat{\mathbf{w}}) & =\int_{\Omega}(\mathbf{f}+\beta \hat{\mathbf{u}}) \cdot \nabla \phi d V \\
& +\sum_{T \in \mathcal{T}} \int_{T}\left(\mathbf{f}+\beta \hat{\mathbf{u}}-\nabla \times \mu^{-1} \nabla \times \hat{\mathbf{u}}\right) \cdot\left(\boldsymbol{\psi}-\mathbf{w}_{2}-\mathbf{w}_{3}\right) d V \\
& -\sum_{T \in \mathcal{T}} \int_{\partial T}\left(\mu^{-1} \nabla \times \hat{\mathbf{u}} \times \mathbf{n}\right) \cdot\left(\boldsymbol{\psi}-\mathbf{w}_{2}\right) d A .
\end{aligned}
$$

Here, we have used the fact that $\mathbf{w}_{3} \times \mathbf{n}=0$ on each face $F \in \mathcal{F}$ to drop it from integral on $\partial T$. Taking $\mathbf{R}_{T}=\mathbf{f}+\beta \hat{\mathbf{u}}-\nabla \times \mu^{-1} \nabla \times \hat{\mathbf{u}}$ on $T$ and $\mathbf{r}_{F}$ to be the jump 
across the interior face $F \in \mathcal{F}$ of $\mu^{-1} \nabla \times \hat{\mathbf{u}}$, and from Lemmas 4.11 and 4.12 , we now have

$$
\begin{aligned}
B(\mathbf{u}-\hat{\mathbf{u}}, \mathbf{v}-\hat{\mathbf{v}}-\hat{\mathbf{w}}) & =\int_{\Omega}(\mathbf{f}+\beta \hat{\mathbf{u}}) \cdot \nabla \phi d V \\
& +\sum_{T \in \mathcal{T}} \int_{T}\left(\mathbf{R}_{T}-\lambda_{T}\right) \cdot\left(\boldsymbol{\psi}-\mathbf{w}_{2}-\mathbf{w}_{3}\right) d V \\
& -\sum_{F \in \mathcal{F}} \int_{F}\left(\left(\mathbf{r}_{F}-\kappa_{F}\right) \times \mathbf{n}\right) \cdot\left(\boldsymbol{\psi}-\mathbf{w}_{2}\right) d A
\end{aligned}
$$

for any constants $\kappa_{F} \in\left[\mathbb{P}_{0}(F)\right]^{3}$ and $\lambda_{T} \in\left[\mathbb{P}_{0}(T)\right]^{3}$.

Next, we apply Lemma 4.14 for the first term and use Cauchy-Schwarz inequality for other terms, we have

$$
\begin{aligned}
|B(\mathbf{u}-\hat{\mathbf{u}}, \mathbf{v}-\hat{\mathbf{v}}-\hat{\mathbf{w}})| & \lesssim h^{1 / 2+\delta}\|\nabla \times(\mathbf{u}-\hat{\mathbf{u}})\|_{L^{2}(\Omega)}\|\mathbf{v}\|_{L^{2}(\Omega)} \\
& +\sum_{T \in \mathcal{T}}\left(\left\|\mathbf{R}_{T}-\lambda_{T}\right\|_{L^{2}(T)}\left(\|\boldsymbol{\psi}\|_{L^{2}(T)}+\left\|\mathbf{w}_{2}\right\|_{L^{2}(T)}+\left\|\mathbf{w}_{3}\right\|_{L^{2}(T)}\right)\right) \\
& +\sum_{F \in \mathcal{F}}\left(\left\|\left(\mathbf{r}_{F}-\kappa_{F}\right) \times \mathbf{n}\right\|_{L^{2}(F)}\left(\|\boldsymbol{\psi}\|_{L^{2}(F)}+\left\|\mathbf{w}_{2}\right\|_{L^{2}(F)}\right)\right)
\end{aligned}
$$

Now, From the results in Theorem 4.9 and Lemmas 4.11 and 4.12 , we obtain

$$
\begin{aligned}
|B(\mathbf{u}-\hat{\mathbf{u}}, \mathbf{v}-\hat{\mathbf{v}}-\hat{\mathbf{w}})| & \lesssim h^{1 / 2+\delta}\|\nabla \times(\mathbf{u}-\hat{\mathbf{u}})\|_{L^{2}(T)}\|\mathbf{v}\|_{L^{2}(\Omega)} \\
& +\sum_{T \in \mathcal{T}} h_{T}\left\|\mathbf{R}_{T}-\lambda_{T}\right\|_{L^{2}(T)}\|\nabla \times \mathbf{v}\|_{L^{2}\left(\tilde{\Omega}_{T}\right)} \\
& +\sum_{F \in \mathcal{F}} h_{F}^{1 / 2}\left\|\left(\mathbf{r}_{F}-\kappa_{F}\right) \times \mathbf{n}\right\|_{L^{2}(F)}\|\nabla \times \mathbf{v}\|_{L^{2}\left(\tilde{\Omega}_{T}\right)} .
\end{aligned}
$$

Since $\|\nabla \times \mathbf{v}\|_{L^{2}\left(\tilde{\Omega}_{T}\right)} \lesssim\|\mathbf{v}\|_{H(\operatorname{curl} ; \Omega)}$ and $\|\mathbf{v}\|_{L^{2}(\Omega)} \lesssim\|\mathbf{v}\|_{H(\operatorname{curl} ; \Omega)}$, and by reordering the right side of the inequality, we get

$$
|B(\mathbf{u}-\hat{\mathbf{u}}, \mathbf{v}-\hat{\mathbf{v}}-\hat{\mathbf{w}})| \lesssim \widetilde{O S C}(\mathbf{u}, \hat{\mathbf{u}}, R, r, \mathcal{T})\|\mathbf{v}\|_{H(\operatorname{curl} ; \Omega)}
$$


where

$$
\begin{aligned}
\widetilde{\mathrm{OSC}}(\mathbf{u}, \hat{\mathbf{u}}, R, r, \mathcal{T}) & =h^{1 / 2+\delta}\|\nabla \times(\mathbf{u}-\hat{\mathbf{u}})\|_{L^{2}(\Omega)}\left\|+\sum_{T \in \mathcal{T}} h_{T} \inf _{\lambda_{\mathbf{T}} \in \mathbb{R}^{\mathbf{3}}}\right\| \mathbf{R}_{T}-\lambda_{T} \|_{L^{2}(T)} \\
& +\sum_{F \in \mathcal{F}} h_{F}^{1 / 2} \inf _{\kappa_{\mathbf{F}} \in \mathbb{R}^{3}}\left\|\left(\mathbf{r}_{F}-\kappa_{F}\right) \times \mathbf{n}\right\|_{L^{2}(F)} .
\end{aligned}
$$

\subsubsection{Proof of Theorem 4.3}

Proof of Theorem 4.3. From Proposition 4.1, we have

$$
|B(\mathbf{u}-\hat{\mathbf{u}}, \mathbf{v})| \leqslant|B(\varepsilon, \hat{\mathbf{w}})|+|B(\mathbf{u}-\hat{\mathbf{u}}, \mathbf{v}-\hat{\mathbf{v}}-\hat{\mathbf{w}})|
$$

Using Cauchy-Schwarz inequality and applying Lemmas 4.11, 4.12, and 4.15 with the fact that $\hat{\mathbf{w}}=\mathbf{w}_{2}+\mathbf{w}_{3}$, we obtain

$$
|B(\mathbf{u}-\hat{\mathbf{u}}, \mathbf{v})| \lesssim\left(\|\varepsilon\|_{H(\operatorname{curl} ; \Omega)}+\widetilde{\mathrm{OSC}}(\mathbf{u}, \hat{\mathbf{u}}, R, r, \mathcal{T})\right)\|\mathbf{v}\|_{H(\operatorname{curl} ; \Omega)}
$$

Now by dividing both sides by $\|\mathbf{v}\|_{H(\operatorname{curl} ; \Omega)}$, we get

$$
\frac{|B(\mathbf{u}-\hat{\mathbf{u}}, \mathbf{v})|}{\|\mathbf{v}\|_{H(\operatorname{curl} ; \Omega)}} \lesssim\left(\|\varepsilon\|_{H(\operatorname{curl} ; \Omega)}+\widetilde{\operatorname{OSc}}(\mathbf{u}, \hat{\mathbf{u}}, R, r, \mathcal{T})\right)
$$

Using the continuous inf-sup condition 2.16 yields

$$
\|\mathbf{u}-\hat{\mathbf{u}}\|_{H(\operatorname{curl} ; \Omega)} \leqslant k_{2}\left(\|\varepsilon\|_{H(\operatorname{curl} ; \Omega)}+\widetilde{\mathrm{OSc}}(\mathbf{u}, \hat{\mathbf{u}}, R, r, \mathcal{T})\right)
$$

where $k_{2}$ is a constant that depends on the shape-regularity of $\mathcal{T}$ and problem data $\mu$ and $\beta$.

When $h$ is sufficiently small then the term $h^{1 / 2+\delta}\|\nabla \times(\mathbf{u}-\hat{\mathbf{u}})\|_{L^{2}(\Omega)} \|$ in the oscillation term $\widetilde{\mathrm{osc}}(\mathbf{u}, \hat{\mathbf{u}}, R, r, \mathcal{T})$ can be absorbed into the left-hand side. Therefore

$$
\|\mathbf{u}-\hat{\mathbf{u}}\|_{H(\operatorname{curl} ; \Omega)} \leqslant k_{2}\left(\|\varepsilon\|_{H(\operatorname{curl} ; \Omega)}+\operatorname{osc}(R, r, \mathcal{T})\right)
$$


where $\operatorname{osc}(R, r, \mathcal{T})$ is the residual oscillation

$$
\operatorname{osc}(R, r, \mathcal{T})^{2}=\sum_{T \in \mathcal{T}} h_{T}^{2} \inf _{\lambda_{\mathbf{T}} \in \mathbb{R}^{\mathbf{3}}}\left\|\mathbf{R}_{T}-\lambda_{T}\right\|_{L^{2}(T)}^{2}+\sum_{F \in \mathcal{F}} h_{F} \inf _{\kappa_{\mathbf{F}} \in \mathbb{R}^{\mathbf{3}}}\left\|\left(\mathbf{r}_{F}-\kappa_{F}\right) \times \mathbf{n}\right\|_{L^{2}(F)}^{2}
$$

This proves the upper bound of the error estimate $\mathbf{u}-\hat{\mathbf{u}}$.

From Proposition 4.8, for any $\tilde{\mathbf{w}} \in W$ and when $h$ is sufficiently small, we have

$$
B(\tilde{\mathbf{w}}, \tilde{\mathbf{w}})=\sum_{T \in \mathcal{T}} B_{T}(\tilde{\mathbf{w}}, \tilde{\mathbf{w}}) \geqslant \sum_{T \in \mathcal{T}} \sigma_{T}\|\nabla \times \tilde{\mathbf{w}}\|_{L^{2}(T)}^{2} \geqslant \sigma\|\nabla \times \tilde{\mathbf{w}}\|_{L^{2}(\Omega)}^{2}
$$

where $\sigma=\min \left\{\sigma_{T}: T \in \mathcal{T}\right\}$. From Proposition 4.7 and the definition of $\|\tilde{\mathbf{w}}\|_{H(\operatorname{curl} ; \Omega)}$, the following hold

$$
\|\tilde{\mathbf{w}}\|_{H(\operatorname{curl} ; \Omega)} \leqslant c\|\nabla \times \tilde{\mathbf{w}}\|_{L^{2}(\Omega)}
$$

Therefore

$$
B(\tilde{\mathbf{w}}, \tilde{\mathbf{w}}) \geqslant \tilde{\sigma}\|\tilde{\mathbf{w}}\|_{H(\operatorname{curl} ; \Omega)}^{2}
$$

where $\tilde{\sigma}=c \sigma$. On the space $W$ and from (4.1), the following hold

$$
|B(\varepsilon, \varepsilon)|=|B(\mathbf{u}-\hat{\mathbf{u}}, \varepsilon)|
$$

Applying the continuity condition, (see section 2.4.4), on the right side and the condition 4.16 on the left side, we obtain

$$
\tilde{\sigma}\|\varepsilon\|_{H(\operatorname{curl} ; \Omega)}^{2} \leqslant|B(\varepsilon, \varepsilon)|=|B(\mathbf{u}-\hat{\mathbf{u}}, \varepsilon)| \leqslant M\|\mathbf{u}-\hat{\mathbf{u}}\|_{H(\operatorname{curl} ; \Omega)}\|\varepsilon\|_{H(\operatorname{curl} ; \Omega)}
$$

therefore

$$
k_{1}\|\varepsilon\|_{H(\operatorname{curl} ; \Omega)} \leqslant\|\mathbf{u}-\hat{\mathbf{u}}\|_{H(\operatorname{curl} ; \Omega)}
$$

where $k_{1}=\frac{\tilde{\sigma}}{M}$. This proves the lower bound of the error estimate $\mathbf{u}-\hat{\mathbf{u}}$ and finish the proof of the theorem. 


\subsection{Computational Cost}

The computation of $\varepsilon$ requires the solution of a global stiffness matrix associated with $W$. So, compared with those implicit methods that are based on computing local (elementwise or patchwise) stiffness matrices, one might ask, for practical computations, which approach is less expensive, solving a single global system or a collection of local systems?. With appropriate auxiliary subspace $W$, we argue that this is not the case and the linear system used to compute the approximate error function has condition number bounded independently of the discretization parameter. More particularly, we argue that the global stiffness matrix for $W$ is spectrally-equivalent to its diagonal, which is not the case for the global stiffness matrix for $V$.

Let $\left\{\psi_{1}, \ldots, \psi_{n}\right\}$ be a basis of a finite dimensional subspace $W \subset \mathcal{H}$, we define the global matrix $B$ by $B_{i j}=B\left(\psi_{j}, \psi_{i}\right)$ and let $D$ be its diagonal. The global stiffness matrix is assembled by summing contributions from element stiffness matrices computed on each simplex. For any $T \in \mathcal{T}$, let $\left\{\phi_{1}, \ldots, \phi_{N}\right\}, N=11$, be a basis of the finite dimensional subspace $W(T) \subset \mathcal{H}$, and in order to show that $B$ and $D$ are spectrally equivalent, we consider the element matrix $B_{T} \in \mathbb{C}^{N \times N}$, defined by $\left(B_{T}\right)_{i j}=B_{T}\left(\phi_{j}, \phi_{i}\right)$, and $D_{T}$ be its diagonal. For any function $\mathbf{v} \in W(T)$, there is a unique representation:

$$
\mathbf{v}=\sum_{i=1}^{N} v_{i} \phi_{i}
$$

where the vector $\boldsymbol{v}=\left\{v_{i}\right\}_{i=1}^{N} \in \mathbb{C}^{N}$ is called the coefficient vector of $\mathbf{v}$ relative to the basis $\left\{\phi_{i}\right\}_{i=1}^{N}$, so we see that

$$
B_{T}(\mathbf{v}, \mathbf{w})=\boldsymbol{w}^{*} B_{T} \boldsymbol{v}, \quad D_{T}(\mathbf{v}, \mathbf{w})=\boldsymbol{w}^{*} D_{T} \boldsymbol{v}=\sum_{k=1}^{N} v_{k} \overline{w_{k}} B_{T}\left(\phi_{k}, \phi_{k}\right) .
$$


From these definitions, we have

$$
\boldsymbol{v}^{*} B \boldsymbol{v}=\sum_{T \in \mathcal{T}} \boldsymbol{v}_{T}^{*} B_{T} \boldsymbol{v}_{T} \quad \text { and } \quad \boldsymbol{v}^{*} D \boldsymbol{v}=\sum_{T \in \mathcal{T}} \boldsymbol{v}_{T}^{*} D_{T} \boldsymbol{v}_{T}
$$

Lemma 4.17. When $h_{T}$ is sufficiently small then $B_{T}(\mathbf{v}, \mathbf{w})$ is an inner product on the subspace $W(T)$.

Proof. From the definition of $B_{T}(\mathbf{v}, \mathbf{w})$, we have

1. For all $\mathbf{v}, \mathbf{w} \in W(T)$ and $\alpha \in \mathbb{C}, B_{T}(\alpha \mathbf{v}, \mathbf{w})=\alpha B_{T}(\mathbf{v}, \mathbf{w})$ and $B_{T}(\mathbf{v}, \alpha \mathbf{w})=$ $\bar{\alpha} B_{T}(\mathbf{v}, \mathbf{w})$. For all $\mathbf{u}, \mathbf{v}, \mathbf{w} \in W(T), B_{T}(\mathbf{u}+\mathbf{v}, \mathbf{w})=B_{T}(\mathbf{u}, \mathbf{w})+B_{T}(\mathbf{v}, \mathbf{w})$ and $B_{T}(\mathbf{u}, \mathbf{v}+\mathbf{w})=B_{T}(\mathbf{u}, \mathbf{v})+B_{T}(\mathbf{u}, \mathbf{w})$ (Additivity)

2. For all $\mathbf{v}, \mathbf{w} \in W(T), B_{T}(\mathbf{w}, \mathbf{v})=\overline{B_{T}(\mathbf{v}, \mathbf{w})}$ (Conjugate symmetry).

For all $\mathbf{v} \in W(T)$ and from Proposition 4.8, when $h_{T}$ is sufficiently small then $B_{T}(\mathbf{v}, \mathbf{v})>\sigma_{T}\|\nabla \times \mathbf{v}\|_{L^{2}(T)}^{2}>0$. Also from Proposition 4.7, $B_{T}(\mathbf{v}, \mathbf{v})=0$ if and only if $\mathbf{v}=0$ (Positive definiteness).

Lemma 4.18. When $h_{T}$ is sufficiently small then $D_{T}(\mathbf{v}, \mathbf{w})$ is an inner product on the subspace $W(T)$.

Proof. The functions $\mathbf{u}, \mathbf{v}$, and $\mathbf{w}$ in $W(T)$ are represented by

$$
\mathbf{u}=\sum_{i=1}^{N} u_{i} \phi_{i}, \mathbf{v}=\sum_{i=1}^{N} v_{i} \phi_{i}, \mathbf{w}=\sum_{i=1}^{N} w_{i} \phi_{i}
$$

where $\boldsymbol{u}=\left\{u_{i}\right\}_{i=1}^{N}, \boldsymbol{v}=\left\{v_{i}\right\}_{i=1}^{N}$, and $\boldsymbol{w}=\left\{w_{i}\right\}_{i=1}^{N}$ in $\mathbb{C}^{N}$ are the coefficient vectors of 
$\mathbf{u}, \mathbf{v}$ and $\mathbf{w}$, respectively. For any $\alpha \in \mathbb{C}$ and from the definition of $D_{T}(\mathbf{v}, \mathbf{w})$, we have

$$
\begin{aligned}
D_{T}(\alpha \mathbf{v}, \mathbf{w}) & =\sum_{k=1}^{N}\left(v_{k} \alpha\right) \overline{w_{k}} B_{T}\left(\phi_{k}, \phi_{k}\right)=\alpha \sum_{k=1}^{N} v_{k} \overline{w_{k}} B_{T}\left(\phi_{k}, \phi_{k}\right) \\
& =\alpha D_{T}(\mathbf{v}, \mathbf{w}), \text { and } \\
D_{T}(\mathbf{v}, \alpha \mathbf{w}) & =\sum_{k=1}^{N} v_{k} \overline{\alpha w_{k}} B_{T}\left(\phi_{k}, \phi_{k}\right)=\bar{\alpha} \sum_{k=1}^{N} v_{k} \overline{w_{k}} B_{T}\left(\phi_{k}, \phi_{k}\right) \\
& =\bar{\alpha} D_{T}(\mathbf{v}, \mathbf{w}) . \\
D_{T}(\mathbf{u}+\mathbf{v}, \mathbf{w})= & \sum_{k=1}^{N}\left(u_{k}+v_{k}\right) \overline{w_{k}} B_{T}\left(\phi_{k}, \phi_{k}\right)=\sum_{k=1}^{N}\left(u_{k} \overline{w_{k}}+v_{k} \overline{w_{k}}\right) B_{T}\left(\phi_{k}, \phi_{k}\right) \\
= & \sum_{k=1}^{N} u_{k} \overline{w_{k}} B_{T}\left(\phi_{k}, \phi_{k}\right)+\sum_{k=1}^{N} v_{k} \overline{w_{k}} B_{T}\left(\phi_{k}, \phi_{k}\right) \\
= & D_{T}(\mathbf{u}, \mathbf{w})+D_{T}(\mathbf{v}, \mathbf{w}), \text { and } \\
D_{T}(\mathbf{u}, \mathbf{v}+\mathbf{w})= & \sum_{k=1}^{N} u_{k}\left(\overline{v_{k}+w_{k}}\right) B_{T}\left(\phi_{k}, \phi_{k}\right)=\sum_{k=1}^{N}\left(u_{k} \overline{v_{k}}+u_{k} \overline{w_{k}}\right) B_{T}\left(\phi_{k}, \phi_{k}\right) \\
= & \sum_{k=1}^{N} u_{k} \overline{v_{k}} B_{T}\left(\phi_{k}, \phi_{k}\right)+\sum_{k=1}^{N} u_{k} \overline{w_{k}} B_{T}\left(\phi_{k}, \phi_{k}\right) \\
= & D_{T}(\mathbf{u}, \mathbf{v})+D_{T}(\mathbf{u}, \mathbf{w}) .
\end{aligned}
$$

This proves the Additivity of $D_{T}(\mathbf{v}, \mathbf{w})$. Also we have

$$
\begin{aligned}
D_{T}(\mathbf{w}, \mathbf{v}) & =\frac{\sum_{k=1}^{N} w_{k} \overline{v_{k}} B_{T}\left(\phi_{k}, \phi_{k}\right)}{=}=\sum_{k=1}^{N} \overline{v_{k} \overline{w_{k}}} \overline{B_{T}\left(\phi_{k}, \phi_{k}\right)} \\
& =\sum_{k=1}^{N} v_{k} \overline{w_{k}} B_{T}\left(\phi_{k}, \phi_{k}\right) \\
& =\overline{D_{T}(\mathbf{v}, \mathbf{w})} .
\end{aligned}
$$

Then the conjugate symmetry condition hold for $D_{T}(\mathbf{v}, \mathbf{w})$. Now from Proposition 4.8, $B_{T}\left(\phi_{k}, \phi_{k}\right)>0$ when $h_{T}$ is sufficiently smal, then $D_{T}(\mathbf{v}, \mathbf{v})>0$ when $\mathbf{v} \neq 0$. This proves the positive definite property and finishes the proof of the lemma. 
The next two results, Lemmas 4.19 and 4.20 , follow immediately from this discussion.

Lemma 4.19. If $B_{T}(\mathbf{v}, \mathbf{w})$ is an inner product on the subspace $W(T)$, then the mapping $\mathbf{v} \longmapsto\langle\langle\mathbf{v}\rangle\rangle_{B_{T}}=\sqrt{\boldsymbol{v}^{*} B_{T} \boldsymbol{v}}=\sqrt{B_{T}(\mathbf{v}, \mathbf{v})}$ is a norm on $W(T)$.

Lemma 4.20. If $D_{T}(\mathbf{v}, \mathbf{w})$ is an inner product on the subspace $W(T)$, then the mapping $\mathbf{v} \longmapsto\langle\langle\mathbf{v}\rangle\rangle_{D_{T}}=\sqrt{\boldsymbol{v}^{*} D_{T} \boldsymbol{v}}=\sqrt{\sum_{k=1}^{N} v_{k} \overline{w_{k}} B_{T}\left(\phi_{k}, \phi_{k}\right)}$ is a norm on $W(T)$.

Lemma 4.21. Let $\mathcal{T}$ be a shape-regular mesh. Then $B_{T}$ and $D_{T}$ are spectrally equivalent, with constants-of-equivalence dependent of the mesh.

Proof. From Lemmas 4.19 and 4.20 , both $\langle\langle\mathbf{v}\rangle\rangle_{B_{T}}$ and $\langle\langle\mathbf{v}\rangle\rangle_{D_{T}}$ are norms on $W(T)$ when $h_{T}$ small enough, so they are equivalent, in other words, there are constants $k_{T}, K_{T}>0$ such that

$$
k_{T} \boldsymbol{v}_{T}^{*} D_{T} \boldsymbol{v}_{T} \leqslant \boldsymbol{v}_{T}^{*} B_{T} \boldsymbol{v}_{T} \leqslant K_{T} \boldsymbol{v}_{T}^{*} D_{T} \boldsymbol{v}_{T}
$$

A scaling argument shows that these constants are scale invariant. Therefore, $B_{T}$ is spectrally equivalent to $D_{T}$.

We are now ready to prove Theorem 4.4, our key result concerning the spectral properties of $B$ for the space $W$ :

\subsubsection{Proof of Theorem 4.4}

Proof of Theorem 4.4. Invoking the shape-regularity of the mesh $\mathcal{T}$, we can replace the local constants $k_{T}, K_{T}$ in Lemma 4.21 with universal constants $k, K$ and apply 4.17), we obtain

$$
k \boldsymbol{v}^{*} D \boldsymbol{v} \leqslant \boldsymbol{v}^{*} B \boldsymbol{v} \leqslant K \boldsymbol{v}^{*} D \boldsymbol{v} \text { for all } \boldsymbol{v} \in \mathbb{C}^{N}
$$

which proves that $B$ and $D$ are spectrally equivalent to each other. 
Remark 4.22. Had we chosen the subspace $V$, and because of the singularity of the local matrix $B_{T}$ in the space $V$, there are no scale-invariant $k_{T}, K_{T}$ for which (4.18) holds. Therefore, Lemma 4.21 cannot be applied.

To provide some sense of what we might expect from constants of equivalence for the spectra of $B_{T}$ and $D_{T}$, we consider a reference tetrahedron for which the spectra is known explicitly.

\subsubsection{Example}

Consider a reference tetrahedron $T$ with side length $h$ having vertices

$$
(0,0,0),(h, 0,0),(0, h, 0),(0,0, h) \text {. }
$$

Element stiffness matrix on $T$ is defined by:

$$
\left(B_{T}\right)_{i j}=\int_{T}\left(\nabla \times \phi_{j}\right) \cdot\left(\nabla \times \overline{\phi_{i}}\right) d V,
$$

and $D_{T}=\operatorname{diag}\left(B_{T}\right)$. From the condition 4.18 we have

$$
K_{T}=\max \frac{\boldsymbol{v}_{T}^{*} B_{T} \boldsymbol{v}_{T}}{\boldsymbol{v}_{T}^{*} D_{T} \boldsymbol{v}_{T}} \quad \text { and } \quad k_{T}=\min \frac{\boldsymbol{v}_{T}^{*} B_{T} \boldsymbol{v}_{T}}{\boldsymbol{v}_{T}^{*} D_{T} \boldsymbol{v}_{T}}
$$

- On $V(T)$, eigenvalues of the $6 \times 6$ element stiffness matrix associated with linear edge bubbles are $\lambda\left(B_{T}\right)=\frac{2}{3 h}\{4,4,1,0,0,0\}$, so $K_{T}=2.5$ and $k_{T}=0$.

This shows that condition 4.18 does not hold and as consequence Lemma 4.21 cannot be applied.

- When $W(T)=W_{2}(T)$, then eigenvalues of the $8 \times 8$ element stiffness matrix associated with quadratic face bubbles are

$$
\lambda\left(B_{T}\right)=h^{-1}\{0.349,0.275,0.128,0.061,0.035,0.028,0.017,0.010\}
$$

so $K_{T}=2.958$ and $k_{T}=0.090$. 
- On $W(T)=W_{2}(T) \oplus W_{3}(T)$, then eigenvalues of the $11 \times 11$ element stiffness matrix for face and interior bubbles are

$$
\begin{gathered}
\lambda\left(B_{T}\right)=h^{-1}\{0.348,0.275,0.128,0.062,0.039,0.031,0.018,0.010,0.003, \\
0.002,0.001\},
\end{gathered}
$$

so $K_{T}=2.994$ and $k_{T}=0.048$.

\subsection{Numerical Experiments}

In this section we will apply the hierarchical basis error estimator that is based on the computation of an approximate error function $\varepsilon$ to some numerical examples. These examples will illustrate the robust effectivity of our proposed estimator function $\varepsilon$.

Our results concerning the reliability and computational cost of our estimator were obtained by performing two refinements techniques, uniform and adaptive hrefinements, on unstructured tetrahedral meshes, using Netgen/NGSolve. In first technique we use the uniform h-refinements method which creates a finer mesh by uniformly decreasing the element size throughout the model. This increases the number of elements until the results stop substantially changing. The second technique is the adaptive h-refinements method. This refines the mesh only in areas containing a high number of errors. The increase in the number of elements that must be processed is substantially less than when uniformly refining the mesh. The technique typically uses an error estimate over the elements at a given solution step and refines the mesh by a practical factor only in the high-error regions and then resolves the problem. The adaptive mesh refinement and resolving continues until reaching the specified accuracy. 
For gauging the quality of the error estimator we use the effectivity index

$$
E=\frac{\text { Estimate error }}{\text { Exact error }}
$$

which gives the ratio between the estimated and the true of discretization error. These reported quantities of effectivities reflect the quality of the global estimate and represent the degree of over or underestimation and should be ideally close to 1 . We will report errors and effectivities in the global $H$-curl norm, because our theory deals with such case, and energy norm, which defined by

$$
\|\mathbf{u}\|^{2}=\left\|\mu^{-1} \nabla \times \mathbf{u}\right\|_{0}^{2}+\|\beta \mathbf{u}\|_{0}^{2}
$$

But as a matter of interest, we also report, in Appendix A, such quantities in global $L^{2}$ norm. Also, we calculated condition numbers for the matrices used to compute $\hat{\mathbf{u}}$ and $\varepsilon$, both with (symmetric) diagonal rescaling.

\subsubsection{Example 1}

In our first experiment, we consider a rather simple case on the unit cube $\Omega=(-1,1)^{3}$ in $3 \mathrm{D}$ with a homogeneous boundary condition that $\mathbf{u}=0$ on $\partial \Omega$. The coefficient $\mu$ is kept constant (equal 1) all over the domain; the coefficient $\beta \in\left\{ \pm 1, \pm 10^{-2}, \pm 10^{-4}\right\}$, and all the computations are conducted on uniformly refined (h-refinements) meshes. The smooth analytic solution $\mathbf{u}$ is given by

$$
\mathbf{u}(x)=\mathbf{u}\left(x_{1}, x_{2}, x_{3}\right)=\left(\begin{array}{l}
1 \\
1 \\
1
\end{array}\right)\left(1-x_{1}^{2}\right)\left(1-x_{2}^{2}\right)\left(1-x_{3}^{2}\right) .
$$

and plotted in Figure 4.2. Then, the load function $f$ can be calculated from (2.9), for the given solution $\mathbf{u}$. 
In the computation, the first grid (coarse mesh) is the given domain with a diagonal line when $\operatorname{Dof}(V)=26$. Then each grid is refined into a half-sized grid uniformly, to get a higher level grid, shown in Figure 4.1. In Figure 4.2, we show a cross sectional view (at $z=0$ ) of the exact solution versus the approximate solution, when $\beta=1$, on different mesh refinements.

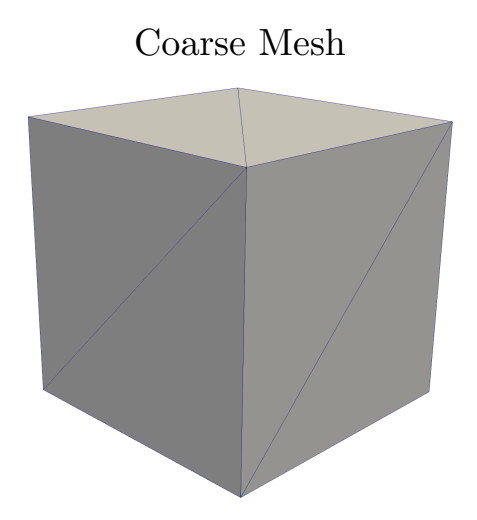

Level 3

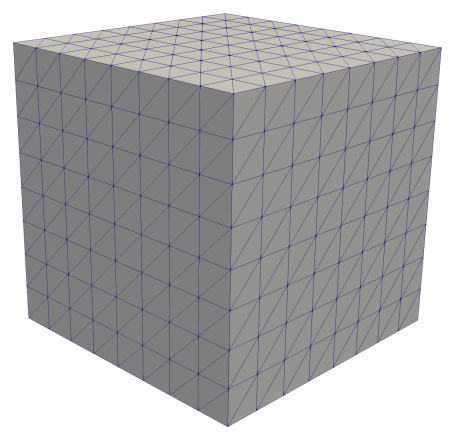

Level 1

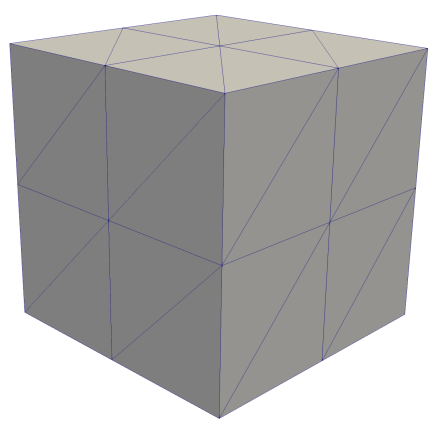

Level 4

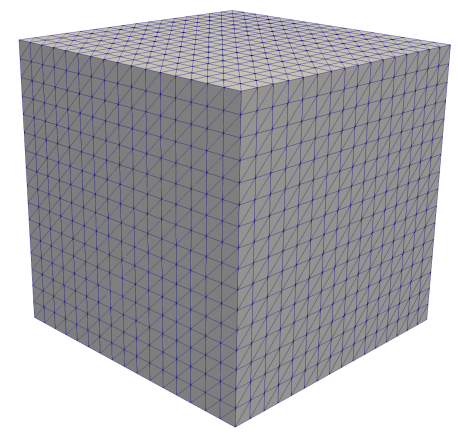

Level 2

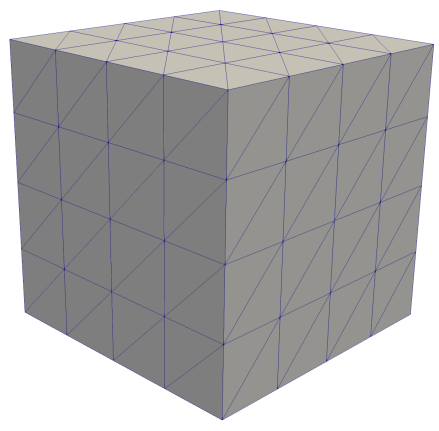

Level 5

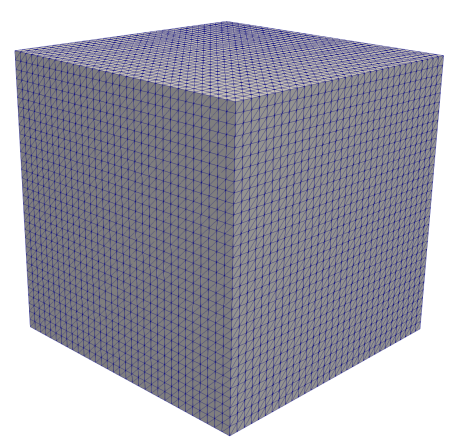

Figure 4.1: The initial mesh and its uniform refinements (Example 1).

Numerical results, in $H$-curl and energy norms, of exact and approximate errors, with convergence order and effectivity indices, are shown in Tables 4.1 and 4.2 for different values of $\beta$. The order of convergence of the method is evaluated by the 


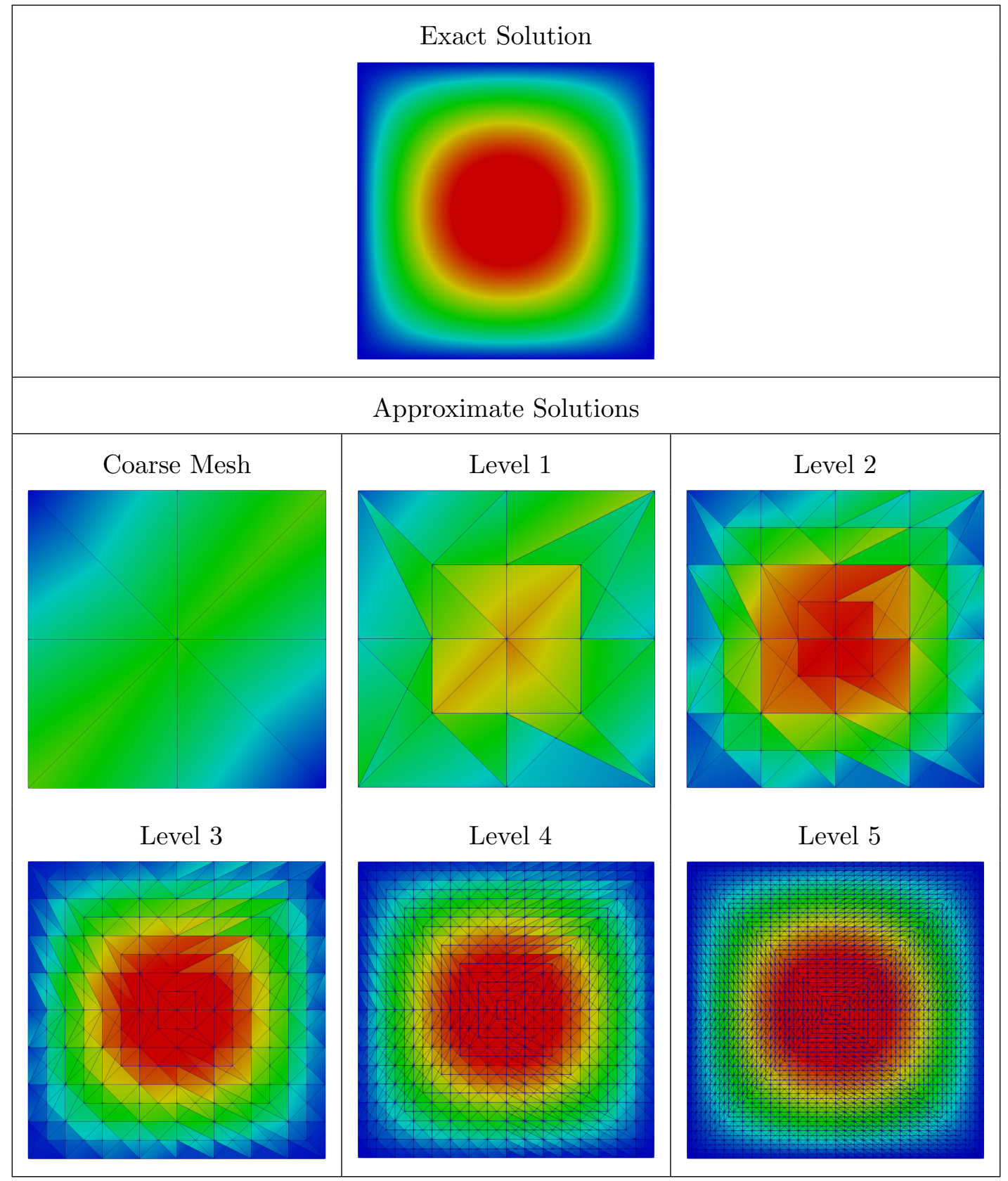

Figure 4.2: Uniform Solutions (Exact Versus Approximate Solutions) (Example 1). 
following formula

$$
\text { order }=\frac{\log \left(E_{i-1} / E_{i}\right)}{\log (2)}
$$

where $E_{i-1}$ and $E_{i}$ are the error norms of numerical solutions for two grid levels. From which, in Figure 4.3, we can see that, for all values of $\beta$, the errors in both norm types are achieve optimal convergence rates $O(h)$ numerically and they as predicted by the theoretical result in Theorem 4.3. The effectivity indices approach a stable constant rapidly as refinement proceeds for most values of $\beta$ as shown in Figure 4.4. For $\beta \in\left\{ \pm 10^{-2}, \pm 10^{-4}\right\}$, the effectivity indices in energy norm reach 0.96 whereas in $H$ curl norm they reach 0.85 . The numerical results for $\beta= \pm 1$ are identical. These results show that our a-posteriori error estimator is efficient and reliable.

In Table 4.3, we present the condition numbers of the diagonally-rescaled stiffness matrices for $W$ and $V$ with respect to the number of degrees of freedom on different mesh refinement levels for positive and negative values of $\beta$. We notice that the computed condition numbers for stiffness matrix for $W$ are stable and bounded in short range [25:96] for most values of $\beta$, see Figure 4.5. However, the condition numbers for stiffness matrix for $V$ grows as the mesh is refined. These results show that the matrix associated with the computation of $\varepsilon$ is much better conditioned, which is certainly not the case for the matrix associated with computing $\hat{\mathbf{u}}$ on $V$. 


\begin{tabular}{|c|c|c|c|c|c|c|}
\hline$\beta$ & $\operatorname{Dof}(V)$ & $\|\mathbf{u}-\hat{\mathbf{u}}\|_{H(\operatorname{curl} ; \Omega)}$ & order & $\|\varepsilon\|_{H(\operatorname{curl} ; \Omega)}$ & order & $\mathrm{EFF}$ \\
\hline \multirow{6}{*}{-1} & 26 & $2.7307 \mathrm{E}+00$ & & $1.4221 \mathrm{E}+00$ & & 0.52 \\
\hline & 180 & $2.0934 \mathrm{E}+00$ & 0.383 & $1.7601 \mathrm{E}+00$ & -0.308 & 0.84 \\
\hline & 1232 & $1.1917 \mathrm{E}+00$ & 0.813 & $1.0155 \mathrm{E}+00$ & 0.794 & 0.85 \\
\hline & 9000 & $6.1597 \mathrm{E}-01$ & 0.952 & $5.2409 \mathrm{E}-01$ & 0.954 & 0.85 \\
\hline & 68696 & $3.1011 \mathrm{E}-01$ & 0.990 & $2.6284 \mathrm{E}-01$ & 0.996 & 0.85 \\
\hline & 536760 & $1.5508 \mathrm{E}-01$ & 1.000 & $1.3109 \mathrm{E}-01$ & 1.004 & 0.85 \\
\hline \multirow{6}{*}{$-10^{-2}$} & 26 & $2.7443 \mathrm{E}+00$ & & $1.5056 \mathrm{E}+00$ & & 0.55 \\
\hline & 180 & $2.1245 \mathrm{E}+00$ & 0.369 & $1.7423 \mathrm{E}+00$ & -0.211 & 0.82 \\
\hline & 1232 & $1.1927 \mathrm{E}+00$ & 0.833 & $1.0143 \mathrm{E}+00$ & 0.780 & 0.85 \\
\hline & 9000 & $6.1610 \mathrm{E}-01$ & 0.953 & $5.2399 \mathrm{E}-01$ & 0.953 & 0.85 \\
\hline & 68696 & $3.1012 \mathrm{E}-01$ & 0.990 & $2.6283 \mathrm{E}-01$ & 0.995 & 0.85 \\
\hline & 536760 & $1.5508 \mathrm{E}-01$ & 1.000 & $1.3108 \mathrm{E}-01$ & 1.004 & 0.85 \\
\hline \multirow{6}{*}{$-10^{-4}$} & 26 & $2.7446 \mathrm{E}+00$ & & $1.5084 \mathrm{E}+00$ & & 0.55 \\
\hline & 180 & $3.4954 \mathrm{E}+01$ & -3.671 & $1.7423 \mathrm{E}+00$ & -0.208 & 0.05 \\
\hline & 1232 & $3.4111 \mathrm{E}+00$ & 3.357 & $1.0144 \mathrm{E}+00$ & 0.780 & 0.30 \\
\hline & 9000 & $6.6006 \mathrm{E}-01$ & 2.370 & $5.2400 \mathrm{E}-01$ & 0.953 & 0.79 \\
\hline & 68696 & $3.1056 \mathrm{E}-01$ & 1.088 & $2.6283 \mathrm{E}-01$ & 0.995 & 0.85 \\
\hline & 536760 & $1.5508 \mathrm{E}-01$ & 1.002 & $1.3108 \mathrm{E}-01$ & 1.004 & 0.85 \\
\hline \multirow{6}{*}{$10^{-}$} & 26 & $2.7446 \mathrm{E}+00$ & & $1.5085 \mathrm{E}+00$ & & 0.55 \\
\hline & 180 & $3.4953 \mathrm{E}+01$ & -3.671 & $1.7423 \mathrm{E}+00$ & -0.208 & 0.05 \\
\hline & 1232 & $3.4110 \mathrm{E}+00$ & 3.357 & $1.0144 \mathrm{E}+00$ & 0.780 & 0.30 \\
\hline & 9000 & $6.6006 \mathrm{E}-01$ & 2.370 & $5.2400 \mathrm{E}-01$ & 0.953 & 0.79 \\
\hline & 68696 & $3.1056 \mathrm{E}-01$ & 1.088 & $2.6283 \mathrm{E}-01$ & 0.995 & 0.85 \\
\hline & 536760 & $1.5508 \mathrm{E}-01$ & 1.002 & $1.3108 \mathrm{E}-01$ & 1.004 & 0.85 \\
\hline \multirow{6}{*}{$10^{-2}$} & 26 & $2.7448 \mathrm{E}+00$ & & $1.5113 \mathrm{E}+00$ & & 0.55 \\
\hline & 180 & $2.1244 \mathrm{E}+00$ & 0.370 & $1.7423 \mathrm{E}+00$ & -0.205 & 0.82 \\
\hline & 1232 & $1.1927 \mathrm{E}+00$ & 0.833 & $1.0144 \mathrm{E}+00$ & 0.780 & 0.85 \\
\hline & 9000 & $6.1610 \mathrm{E}-01$ & 0.953 & $5.2400 \mathrm{E}-01$ & 0.953 & 0.85 \\
\hline & 68696 & $3.1013 \mathrm{E}-01$ & 0.990 & $2.6283 \mathrm{E}-01$ & 0.995 & 0.85 \\
\hline & 536760 & $1.5508 \mathrm{E}-01$ & 1.000 & $1.3108 \mathrm{E}-01$ & 1.004 & 0.85 \\
\hline & 26 & $2.7880 \mathrm{E}+00$ & & $2.2035 \mathrm{E}+00$ & & 0.79 \\
\hline & 180 & $2.1044 \mathrm{E}+00$ & 0.406 & $1.7765 \mathrm{E}+00$ & 0.311 & 0.84 \\
\hline & 1232 & $1.1946 \mathrm{E}+00$ & 0.817 & $1.0221 \mathrm{E}+00$ & 0.797 & 0.86 \\
\hline & 9000 & $6.1652 \mathrm{E}-01$ & 0.954 & $5.2519 \mathrm{E}-01$ & 0.961 & 0.85 \\
\hline & 68696 & $3.1019 \mathrm{E}-01$ & 0.991 & $2.6299 \mathrm{E}-01$ & 0.998 & 0.85 \\
\hline & 536760 & $1.5509 \mathrm{E}-01$ & 1.000 & $1.3111 \mathrm{E}-01$ & 1.004 & 0.85 \\
\hline
\end{tabular}

Table 4.1: Numerical errors, convergence order, and effectivity indices of the error estimator in $H$-curl norm (Example 1). 


\begin{tabular}{c||l|cc|cc|c}
\hline$\beta$ & $D o f(V)$ & $\|\mathbf{u}-\hat{\mathbf{u}}\|$ & order & $\|\varepsilon\|$ & order & EFF \\
\hline \multirow{5}{*}{-1} & 26 & $2.7307 \mathrm{E}+00$ & & $1.4221 \mathrm{E}+00$ & & 0.52 \\
& 180 & $2.0934 \mathrm{E}+00$ & 0.383 & $1.7601 \mathrm{E}+00$ & -0.308 & 0.84 \\
& 1232 & $1.1917 \mathrm{E}+00$ & 0.813 & $1.0155 \mathrm{E}+00$ & 0.794 & 0.85 \\
& 9000 & $6.1597 \mathrm{E}-01$ & 0.952 & $5.2409 \mathrm{E}-01$ & 0.954 & 0.85 \\
& 68696 & $3.1011 \mathrm{E}-01$ & 0.990 & $2.6284 \mathrm{E}-01$ & 0.996 & 0.85 \\
& 536760 & $1.5508 \mathrm{E}-01$ & 1.000 & $1.3109 \mathrm{E}-01$ & 1.004 & 0.85 \\
\hline \multirow{5}{*}{$-10^{-2}$} & 26 & $2.2662 \mathrm{E}+00$ & & $1.4631 \mathrm{E}+00$ & & 0.65 \\
& 180 & $1.8593 \mathrm{E}+00$ & 0.285 & $1.7104 \mathrm{E}+00$ & -0.225 & 0.92 \\
& 1232 & $1.0616 \mathrm{E}+00$ & 0.808 & $1.0089 \mathrm{E}+00$ & 0.762 & 0.95 \\
& 9000 & $5.4552 \mathrm{E}-01$ & 0.961 & $5.2328 \mathrm{E}-01$ & 0.947 & 0.96 \\
& 68696 & $2.7356 \mathrm{E}-01$ & 0.996 & $2.6274 \mathrm{E}-01$ & 0.994 & 0.96 \\
& 536760 & $1.3655 \mathrm{E}-01$ & 1.002 & $1.3107 \mathrm{E}-01$ & 1.003 & 0.96 \\
\hline \multirow{5}{*}{$10^{-4}$} & 26 & $2.2610 \mathrm{E}+00$ & & $1.4649 \mathrm{E}+00$ & & 0.65 \\
& 180 & $1.8890 \mathrm{E}+00$ & 0.259 & $1.7101 \mathrm{E}+00$ & -0.223 & 0.91 \\
& 1232 & $1.0607 \mathrm{E}+00$ & 0.833 & $1.0089 \mathrm{E}+00$ & 0.761 & 0.95 \\
& 9000 & $5.4477 \mathrm{E}-01$ & 0.961 & $5.2327 \mathrm{E}-01$ & 0.947 & 0.96 \\
& 68696 & $2.7317 \mathrm{E}-01$ & 0.996 & $2.6274 \mathrm{E}-01$ & 0.994 & 0.96 \\
& 536760 & $1.3636 \mathrm{E}-01$ & 1.002 & $1.3107 \mathrm{E}-01$ & 1.003 & 0.96 \\
\hline \multirow{5}{*}{$10^{-2}$} & 26 & $2.2610 \mathrm{E}+00$ & & $1.4650 \mathrm{E}+00$ & & 0.65 \\
& 180 & $1.8890 \mathrm{E}+00$ & 0.259 & $1.7101 \mathrm{E}+00$ & -0.223 & 0.91 \\
& 1232 & $1.0607 \mathrm{E}+00$ & 0.833 & $1.0089 \mathrm{E}+00$ & 0.761 & 0.95 \\
& 9000 & $5.4477 \mathrm{E}-01$ & 0.961 & $5.2327 \mathrm{E}-01$ & 0.947 & 0.96 \\
& 68696 & $2.7317 \mathrm{E}-01$ & 0.996 & $2.6274 \mathrm{E}-01$ & 0.994 & 0.96 \\
& 536760 & $1.3636 \mathrm{E}-01$ & 1.002 & $1.3107 \mathrm{E}-01$ & 1.003 & 0.96 \\
\hline \multirow{5}{*}{} & 26 & $2.2663 \mathrm{E}+00$ & & $1.4677 \mathrm{E}+00$ & & 0.65 \\
& 1232 & $1.8593 \mathrm{E}+00$ & 0.286 & $1.7104 \mathrm{E}+00$ & -0.221 & 0.92 \\
& 9000 & $1.0616 \mathrm{E}+00$ & 0.808 & $1.0089 \mathrm{E}+00$ & 0.762 & 0.95 \\
& $5.4552 \mathrm{E}-01$ & 0.961 & $5.2329 \mathrm{E}-01$ & 0.947 & 0.96 \\
& 536760 & $2.7356 \mathrm{E}-01$ & 0.996 & $2.6274 \mathrm{E}-01$ & 0.994 & 0.96 \\
& 26 & $1.3655 \mathrm{E}-01$ & 1.002 & $1.3107 \mathrm{E}-01$ & 1.003 & 0.96 \\
\hline & 180 & $2.7880 \mathrm{E}+00$ & & $2.2035 \mathrm{E}+00$ & & 0.79 \\
& 5000 & $6.1044 \mathrm{E}+00$ & 0.406 & $1.7765 \mathrm{E}+00$ & 0.311 & 0.84 \\
& 536760 & $1.5509 \mathrm{E}-01$ & 1.000 & $1.3111 \mathrm{E}-01$ & 1.004 & 0.85 \\
\hline
\end{tabular}

Table 4.2: Numerical errors, convergence order, and effectivity indices of the error estimator in energy norm (Example 1). 


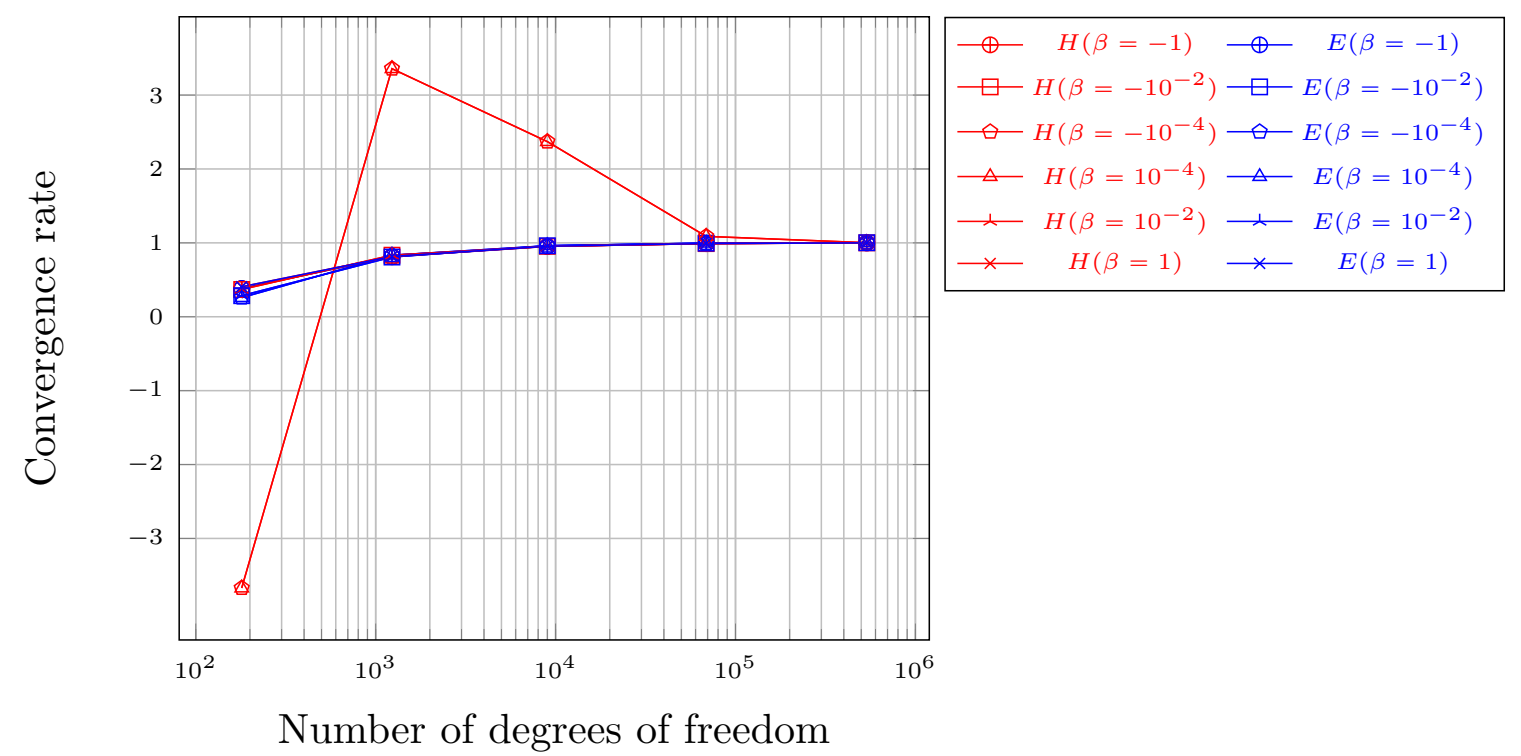

Figure 4.3: Convergence rate of the exact error in $H$-curl norm (red) and energy norm (blue) (Example 1).

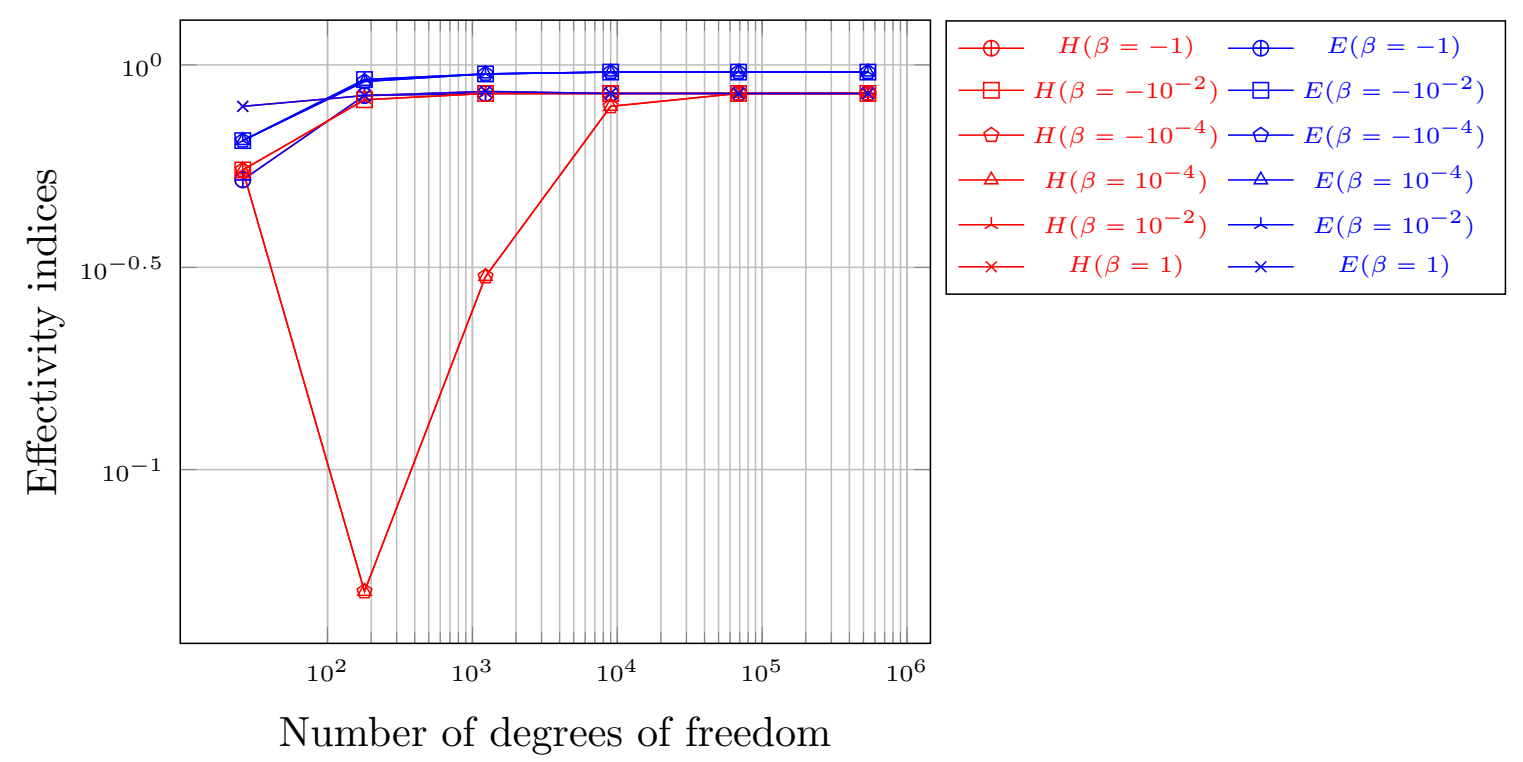

Figure 4.4: Effectivity indices of the error estimators in $H$-curl norm (red) and energy norm (blue) (Example 1). 


\begin{tabular}{|c|c|c|c|}
\hline$\beta$ & $\operatorname{Dof}(V)$ & Cnd. No $(V)$ & Cnd. No $(W)$ \\
\hline \multirow{6}{*}{-1} & 26 & 8.706 & 25.655 \\
\hline & 180 & 64.518 & 57.751 \\
\hline & 1232 & 343.103 & 83.063 \\
\hline & 9000 & 1188.105 & 90.886 \\
\hline & 68696 & 4221.520 & 94.566 \\
\hline & 536760 & 15901.549 & 94.991 \\
\hline \multirow{6}{*}{$-10^{-2}$} & 26 & 800.711 & 35.898 \\
\hline & 180 & 5553.342 & 63.707 \\
\hline & 1232 & 21701.830 & 84.140 \\
\hline & 9000 & 88097.925 & 91.199 \\
\hline & 68696 & 121484.607 & 94.650 \\
\hline & 536760 & 174241.066 & 95.012 \\
\hline \multirow{6}{*}{$-10^{-4}$} & 26 & 80000.711 & 36.072 \\
\hline & 180 & 419178.450 & 63.779 \\
\hline & 1232 & 2160434.527 & 84.151 \\
\hline & 9000 & 720265.625 & 91.202 \\
\hline & 68696 & 170300.780 & 94.651 \\
\hline & 536760 & 193680.976 & 95.012 \\
\hline \multirow{6}{*}{$10^{-4}$} & 26 & 79999.289 & 36.076 \\
\hline & 180 & 422578.963 & 63.754 \\
\hline & 1232 & 2213695.104 & 80.113 \\
\hline & 9000 & 915037.942 & 90.301 \\
\hline & 68696 & 169107.658 & 93.435 \\
\hline & 536760 & 194118.539 & 95.012 \\
\hline \multirow{6}{*}{$10^{-2}$} & 26 & 799.289 & 36.252 \\
\hline & 180 & 4132.533 & 63.827 \\
\hline & 1232 & 22021.853 & 80.125 \\
\hline & 9000 & 111247.673 & 90.304 \\
\hline & 68696 & 281605.782 & 93.436 \\
\hline & 536760 & 218562.354 & 95.013 \\
\hline \multirow{6}{*}{1} & 26 & 7.279 & 89.649 \\
\hline & 180 & 36.277 & 72.875 \\
\hline & 1232 & 166.470 & 81.341 \\
\hline & 9000 & 837.108 & 90.644 \\
\hline & 68696 & 4142.714 & 93.522 \\
\hline & 536760 & 18690.529 & 95.034 \\
\hline
\end{tabular}

Table 4.3: Condition numbers of the stiffness matrices for $V$ and $W$ (Example 1). 


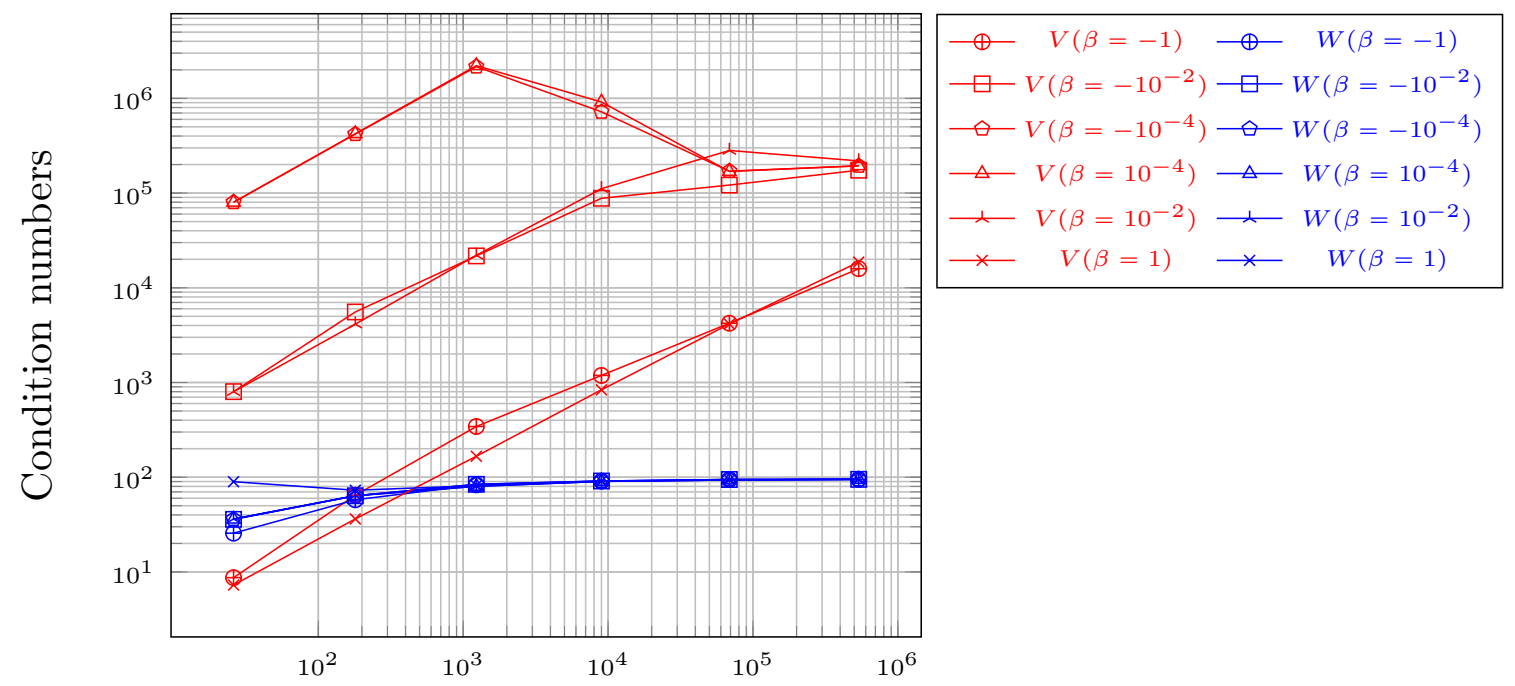

Number of degrees of freedom

Figure 4.5: Condition numbers of the stiffness matrices for $V$ (red) and $W$ (blue) (Example 1).

\subsubsection{Example 2}

In this example, we solve the equation 2.9 on a unit cube $\Omega=(-1,1)^{3}$ with a homogeneous boundary condition that $\mathbf{u}=0$ on $\partial \Omega$. As before, we keep the coefficient $\mu$ constant (equal 1) all over the domain but the values of the coefficient $\beta$ are $\pm 10^{-2}$ and \pm 1 . Here, we consider the particular problem where the exact solution $\mathbf{u}$ is given by the function

$$
\mathbf{u}(x)=\mathbf{u}\left(x_{1}, x_{2}, x_{3}\right)=\left(\begin{array}{l}
1 \\
1 \\
1
\end{array}\right)\left(1-x_{1}^{2}\right)\left(1-x_{2}^{2}\right)\left(1-x_{3}^{2}\right)\left(x_{1}^{2}+x_{2}^{2}+x_{3}^{2}+10^{-2}\right)^{-0.4} .
$$

The load function $f$ can be calculated exactly from $(2.9)$, for the given solution $\mathbf{u}$. In this example, we will apply the adaptive h-refinements method, and due to the behavior of $\mathbf{u}(x)$ at the origin, we expect to require most refinement near the origin. 
We start our procedure with a coarse Delaunay triangulation. A cross sectional view (at $z=0)$ of the initial coarse mesh $\left(\operatorname{DoF}(V) N_{o}=26\right)$ along with the adaptively refined grids, for $\beta=1$, are plotted in Figure 4.6. We observe that, as the meshes are adaptively refined, they become much denser close to the origin due to the singularity of $\mathbf{u}$. In Figure 4.7, a cross sectional view (at $z=0$ ) of the exact solution and the approximate solution are showen for $\beta=1$, on different mesh levels. 
Coarse Mesh

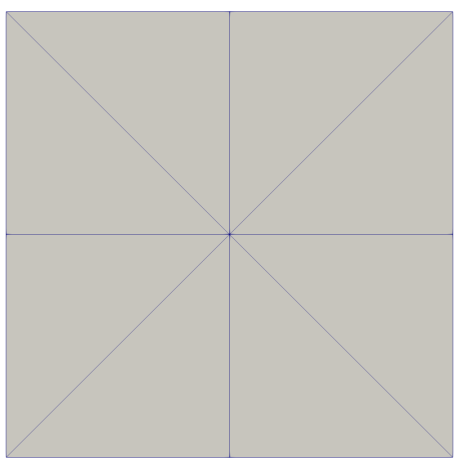

Level 3

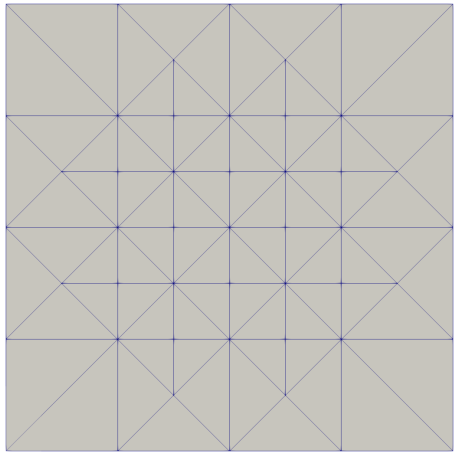

Level 6

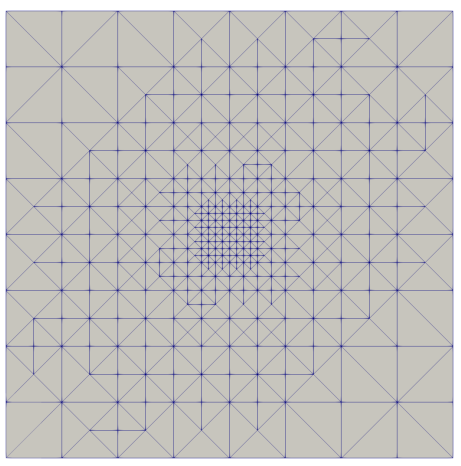

Level 1

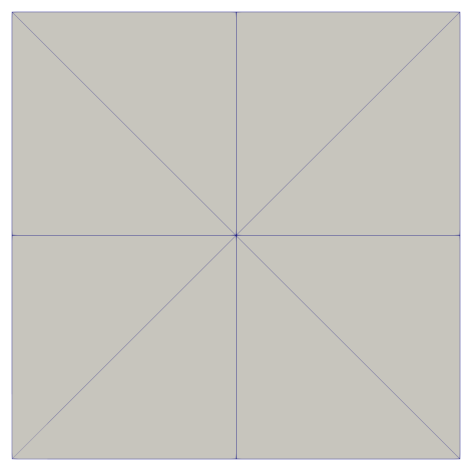

Level 4

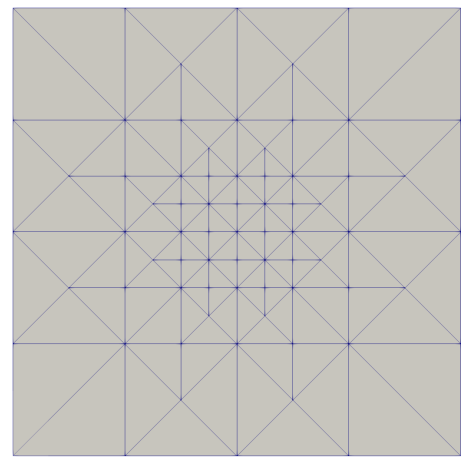

Level 7

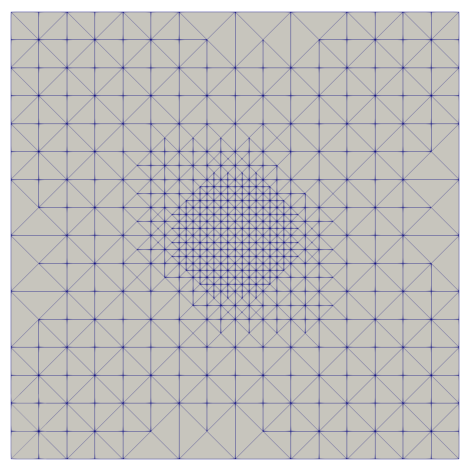

Level 2

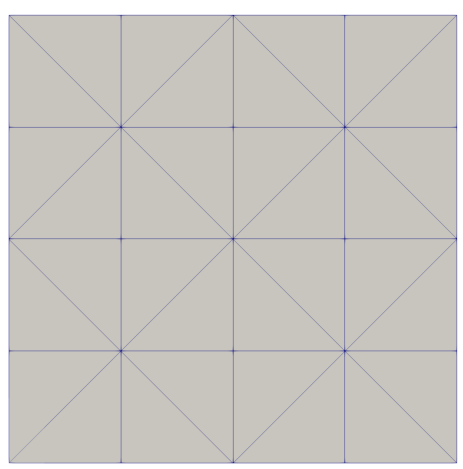

Level 5

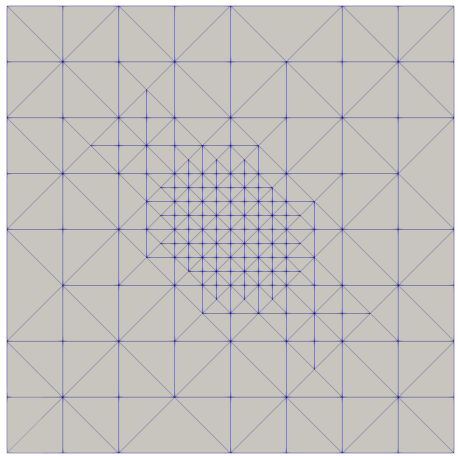

Figure 4.6: Coarse mesh and Adaptive Refinement Levels (Example 2). 


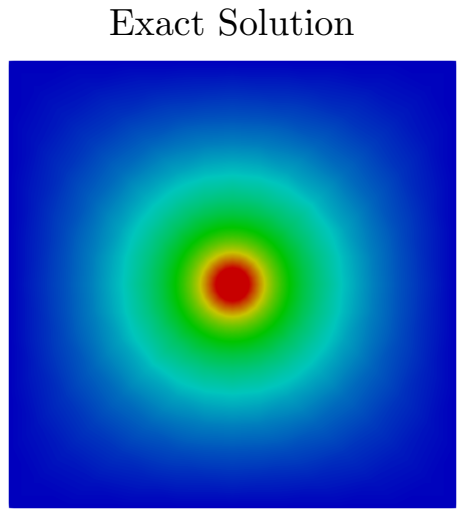

Level 2

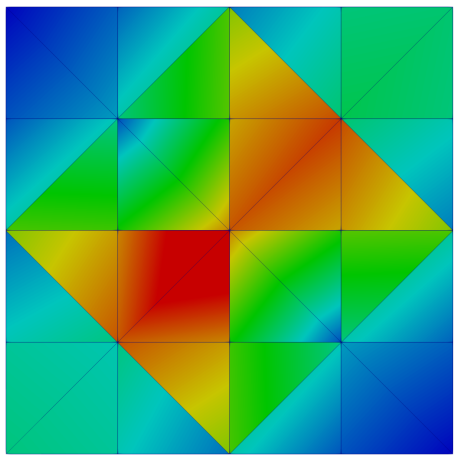

Level 5

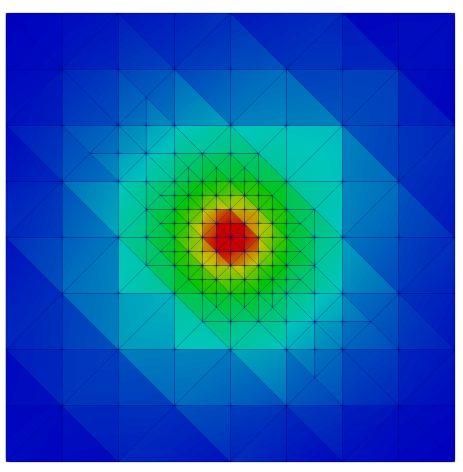

Coarse Mesh

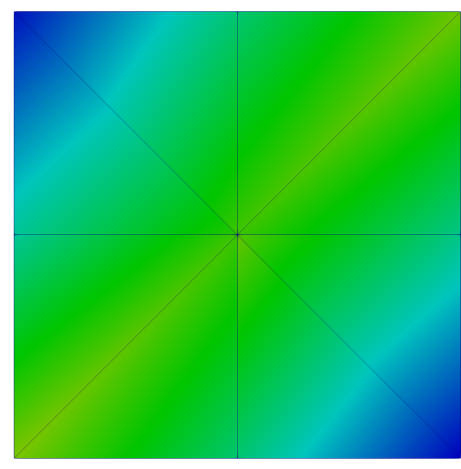

Level 3

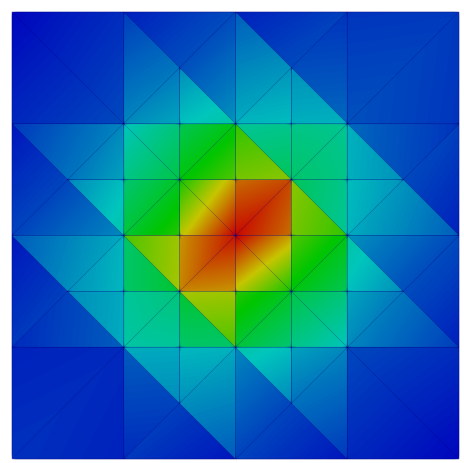

Level 6

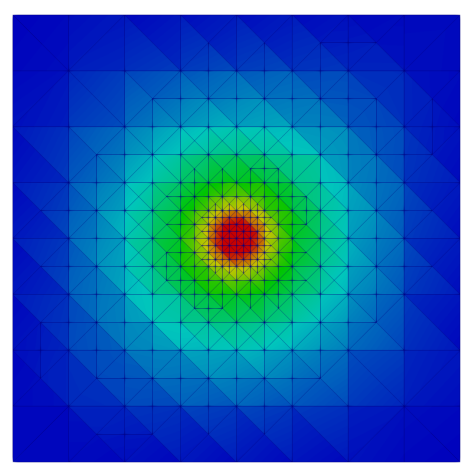

Level 1

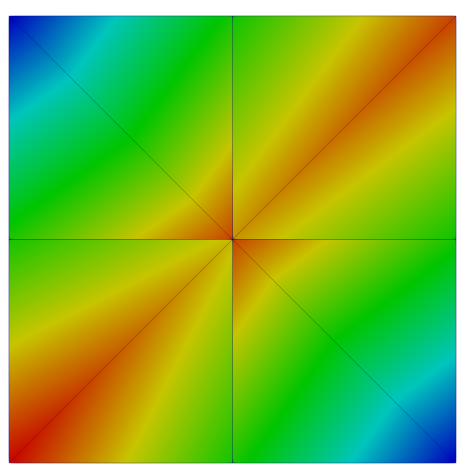

Level 4

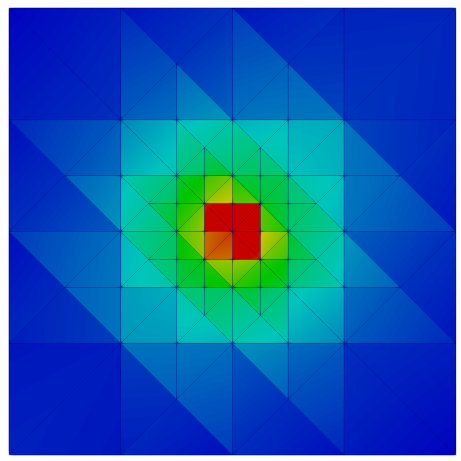

Level 7

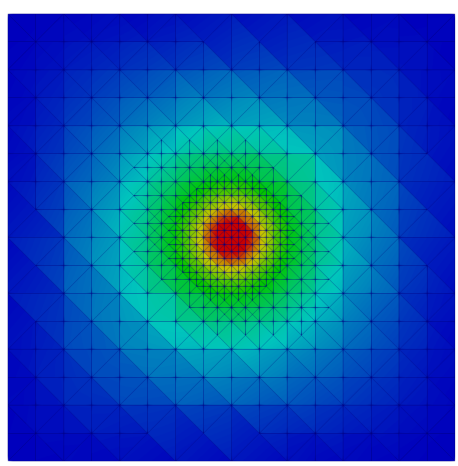

Figure 4.7: Adaptive Solutions (Exact Versus Approximate Solutions) (Example 2).

In Tables 4.4 and 4.5, the errors (exact and approximate errors), convergence order, and effectivity indices, in $H$-curl and energy norms, respectively, are listed for 
different values of $\beta$. Here, we define the convergence order, with respect to $\operatorname{dim}(V)$, by the formula

$$
\text { order }=\frac{\log \left(E_{i-1} / E_{i}\right)}{\log \left(N_{i-1} / N_{i}\right)}
$$

where $E_{i-1}$ and $E_{i}$ are the error norms of numerical solutions for two grid levels and $N_{i-1}$ and $N_{i}$ are $\operatorname{dim}(V)$ on these grids.

Remark 4.23. For the space V (1-order Nédélec space of the first-kind), we have $\operatorname{dim}(V)=\operatorname{Dof}(V)$.

From the data in these tables and as expected, we observe that, for refinement level sufficiently large, the approximate errors in both norm types are achieve optimal convergence rates, approximately $O\left(\operatorname{dim}(V)^{-1 / 3}\right)$, which coincides with our theory, see Figure 4.8. For $\beta= \pm 1$ and as showen in Figure 4.9, the effectivity indices fastly approach a stable value which is not the case when $\beta= \pm 10^{-2}$, where these effectivities reach a stable limit on finer discretization.

The condition numbers of the diagonally-rescaled stiffness matrices for $W$ and $V$, with respect to the number of degrees of freedom, are showen in Table 4.6 for all values of $\beta$. In most cases of $\beta$ values and all mesh levels, we can see, in Figure 4.10 , that the computed condition numbers for $W$ remained in a relatively narrow range, neither monotonically increasing nor decreasing as the mesh was refined. The range of condition numbers was $[25,90]$. However, the condition numbers for stiffness matrix for $V$ are growing as the mesh is adaptively refined, and their ranges vary based on the values of $\beta$. 


\begin{tabular}{|c|c|c|c|c|c|c|}
\hline$\beta$ & $\operatorname{Dof}(V)$ & $\|\mathbf{u}-\hat{\mathbf{u}}\|_{H(\operatorname{curl} ; \Omega)}$ & order & $\|\varepsilon\|_{H(\operatorname{curl} ; \Omega)}$ & order & $\mathrm{EFF}$ \\
\hline \multirow{8}{*}{-1} & 26 & $7.6391 \mathrm{E}+00$ & & $3.4378 \mathrm{E}+00$ & & 0.45 \\
\hline & 180 & $6.5604 \mathrm{E}+00$ & -0.079 & $3.5088 \mathrm{E}+00$ & 0.011 & 0.53 \\
\hline & 1216 & $5.1082 \mathrm{E}+00$ & -0.131 & $2.9435 \mathrm{E}+00$ & -0.092 & 0.58 \\
\hline & 2852 & $2.8118 \mathrm{E}+00$ & -0.700 & $2.3723 \mathrm{E}+00$ & -0.253 & 0.84 \\
\hline & 4552 & $2.2017 \mathrm{E}+00$ & -0.523 & $1.9518 \mathrm{E}+00$ & -0.417 & 0.89 \\
\hline & 17076 & $1.4952 \mathrm{E}+00$ & -0.293 & $1.3461 \mathrm{E}+00$ & -0.281 & 0.90 \\
\hline & 45298 & $1.0316 \mathrm{E}+00$ & -0.380 & $9.5316 \mathrm{E}-01$ & -0.354 & 0.92 \\
\hline & 94716 & $8.3278 \mathrm{E}-01$ & -0.290 & $7.6250 \mathrm{E}-01$ & -0.303 & 0.92 \\
\hline \multirow{8}{*}{$-10^{-2}$} & 26 & $7.6259 \mathrm{E}+00$ & & $4.7063 \mathrm{E}+00$ & & 0.62 \\
\hline & 180 & $1.0510 \mathrm{E}+02$ & 1.356 & $4.1530 \mathrm{E}+00$ & -0.065 & 0.04 \\
\hline & 1216 & $2.0992 \mathrm{E}+02$ & 0.362 & $3.0724 \mathrm{E}+00$ & -0.158 & 0.01 \\
\hline & 2852 & $2.4612 \mathrm{E}+01$ & -2.514 & $2.3921 \mathrm{E}+00$ & -0.294 & 0.10 \\
\hline & 4552 & $4.3938 \mathrm{E}+01$ & 1.240 & $1.9562 \mathrm{E}+00$ & -0.430 & 0.04 \\
\hline & 15708 & $3.5598 \mathrm{E}+00$ & -2.029 & $1.4050 \mathrm{E}+00$ & -0.267 & 0.39 \\
\hline & 32368 & $1.2959 \mathrm{E}+00$ & -1.398 & $1.0539 \mathrm{E}+00$ & -0.398 & 0.81 \\
\hline & 103786 & 8.4243E-01 & -0.370 & $7.3429 \mathrm{E}-01$ & -0.310 & 0.87 \\
\hline \multirow{8}{*}{$10^{-2}$} & 26 & $7.6256 \mathrm{E}+00$ & & $4.7400 \mathrm{E}+00$ & & 0.62 \\
\hline & 180 & $1.0510 \mathrm{E}+02$ & 1.356 & $4.1688 \mathrm{E}+00$ & -0.066 & 0.04 \\
\hline & 1216 & $2.0992 \mathrm{E}+02$ & 0.362 & $3.0752 \mathrm{E}+00$ & -0.159 & 0.01 \\
\hline & 2852 & $2.4614 \mathrm{E}+01$ & -2.514 & $2.3925 \mathrm{E}+00$ & -0.294 & 0.10 \\
\hline & 4552 & $4.3937 \mathrm{E}+01$ & 1.239 & $1.9563 \mathrm{E}+00$ & -0.430 & 0.04 \\
\hline & 15708 & $3.5598 \mathrm{E}+00$ & -2.029 & $1.4051 \mathrm{E}+00$ & -0.267 & 0.39 \\
\hline & 32368 & $1.2959 \mathrm{E}+00$ & -1.398 & $1.0540 \mathrm{E}+00$ & -0.398 & 0.81 \\
\hline & 103786 & $8.4242 \mathrm{E}-01$ & -0.370 & $7.3429 \mathrm{E}-01$ & -0.310 & 0.87 \\
\hline \multirow{8}{*}{1} & 26 & $7.6104 \mathrm{E}+00$ & & $7.1417 \mathrm{E}+00$ & & 0.94 \\
\hline & 180 & $6.5120 \mathrm{E}+00$ & -0.081 & $5.1375 \mathrm{E}+00$ & -0.170 & 0.79 \\
\hline & 1168 & $5.1718 \mathrm{E}+00$ & -0.123 & $3.2377 \mathrm{E}+00$ & -0.247 & 0.63 \\
\hline & 2804 & $2.8349 \mathrm{E}+00$ & -0.686 & $2.4408 \mathrm{E}+00$ & -0.323 & 0.86 \\
\hline & 4504 & $2.2319 \mathrm{E}+00$ & -0.505 & $1.9949 \mathrm{E}+00$ & -0.426 & 0.89 \\
\hline & 15048 & $1.5689 \mathrm{E}+00$ & -0.292 & $1.4232 \mathrm{E}+00$ & -0.280 & 0.91 \\
\hline & 31852 & $1.1565 \mathrm{E}+00$ & -0.407 & $1.0633 \mathrm{E}+00$ & -0.389 & 0.92 \\
\hline & 95494 & 8.2029E-01 & -0.313 & $7.5384 \mathrm{E}-01$ & -0.313 & 0.92 \\
\hline
\end{tabular}

Table 4.4: Numerical errors, convergence order, and effectivity indices of the error estimator in $H$-curl norm (Example 2). 


\begin{tabular}{|c|c|c|c|c|c|c|}
\hline$\beta$ & $\operatorname{Dof}(V)$ & $\|\mathbf{u}-\hat{\mathbf{u}}\|$ & order & $\|\varepsilon\|$ & order & $\mathrm{EFF}$ \\
\hline \multirow{8}{*}{-1} & 26 & $7.6391 \mathrm{E}+00$ & & $3.4378 \mathrm{E}+00$ & & 0.45 \\
\hline & 180 & $6.5604 \mathrm{E}+00$ & -0.079 & $3.5088 \mathrm{E}+00$ & 0.011 & 0.53 \\
\hline & 1216 & $5.1082 \mathrm{E}+00$ & -0.131 & $2.9435 \mathrm{E}+00$ & -0.092 & 0.58 \\
\hline & 2852 & $2.8118 \mathrm{E}+00$ & -0.700 & $2.3723 \mathrm{E}+00$ & -0.253 & 0.84 \\
\hline & 4552 & $2.2017 \mathrm{E}+00$ & -0.523 & $1.9518 \mathrm{E}+00$ & -0.417 & 0.89 \\
\hline & 17076 & $1.4952 \mathrm{E}+00$ & -0.293 & $1.3461 \mathrm{E}+00$ & -0.281 & 0.90 \\
\hline & 45298 & $1.0316 \mathrm{E}+00$ & -0.380 & $9.5316 \mathrm{E}-01$ & -0.354 & 0.92 \\
\hline & 94716 & $8.3278 \mathrm{E}-01$ & -0.290 & $7.6250 \mathrm{E}-01$ & -0.303 & 0.92 \\
\hline \multirow{8}{*}{$-10^{-}$} & 26 & $7.0392 \mathrm{E}+00$ & & $4.3636 \mathrm{E}+00$ & & 0.62 \\
\hline & 180 & $1.2062 \mathrm{E}+01$ & 0.278 & $4.0140 \mathrm{E}+00$ & -0.043 & 0.33 \\
\hline & 1216 & $2.1456 \mathrm{E}+01$ & 0.301 & $3.0488 \mathrm{E}+00$ & -0.144 & 0.14 \\
\hline & 2852 & $3.6273 \mathrm{E}+00$ & -2.085 & $2.3878 \mathrm{E}+00$ & -0.287 & 0.66 \\
\hline & 4552 & $4.8423 \mathrm{E}+00$ & 0.618 & $1.9533 \mathrm{E}+00$ & -0.430 & 0.40 \\
\hline & 15708 & $1.4940 \mathrm{E}+00$ & -0.949 & $1.4037 \mathrm{E}+00$ & -0.267 & 0.94 \\
\hline & 32368 & $1.0892 \mathrm{E}+00$ & -0.437 & $1.0535 \mathrm{E}+00$ & -0.397 & 0.97 \\
\hline & 1037 & $7.5752 \mathrm{E}-01$ & -0.312 & 7.3413E-01 & -0.310 & 0.97 \\
\hline \multirow{8}{*}{$10^{-2}$} & 26 & $7.0388 \mathrm{E}+00$ & & $4.3933 \mathrm{E}+00$ & & 0.62 \\
\hline & 180 & $1.2062 \mathrm{E}+01$ & 0.278 & $4.0287 \mathrm{E}+00$ & -0.045 & 0.33 \\
\hline & 1216 & $2.1457 \mathrm{E}+01$ & 0.301 & $3.0516 \mathrm{E}+00$ & -0.145 & 0.14 \\
\hline & 2852 & $3.6274 \mathrm{E}+00$ & -2.085 & $2.3882 \mathrm{E}+00$ & -0.288 & 0.66 \\
\hline & 4552 & $4.8423 \mathrm{E}+00$ & 0.618 & $1.9534 \mathrm{E}+00$ & -0.430 & 0.40 \\
\hline & 15708 & $1.4940 \mathrm{E}+00$ & -0.949 & $1.4038 \mathrm{E}+00$ & -0.267 & 0.94 \\
\hline & 32368 & $1.0892 \mathrm{E}+00$ & -0.437 & $1.0535 \mathrm{E}+00$ & -0.397 & 0.97 \\
\hline & 103786 & $7.5752 \mathrm{E}-01$ & -0.312 & $7.3414 \mathrm{E}-01$ & -0.310 & 0.97 \\
\hline & 26 & $7.6104 \mathrm{E}+00$ & & $7.1417 \mathrm{E}+00$ & & 0.94 \\
\hline & 180 & $6.5120 \mathrm{E}+00$ & -0.081 & $5.1375 \mathrm{E}+00$ & -0.170 & 0.79 \\
\hline & 1168 & $5.1718 \mathrm{E}+00$ & -0.123 & $3.2377 \mathrm{E}+00$ & -0.247 & 0.63 \\
\hline & 2804 & $2.8349 \mathrm{E}+00$ & -0.686 & $2.4408 \mathrm{E}+00$ & -0.323 & 0.86 \\
\hline & 4504 & $2.2319 \mathrm{E}+00$ & -0.505 & $1.9949 \mathrm{E}+00$ & -0.426 & 0.89 \\
\hline & 15048 & $1.5689 \mathrm{E}+00$ & -0.292 & $1.4232 \mathrm{E}+00$ & -0.280 & 0.91 \\
\hline & 31852 & $1.1565 \mathrm{E}+00$ & -0.407 & $1.0633 \mathrm{E}+00$ & -0.389 & 0.92 \\
\hline & 95494 & $8.2029 \mathrm{E}-01$ & -0.313 & 7.5384E-01 & -0.313 & 0.92 \\
\hline
\end{tabular}

Table 4.5: Numerical errors, convergence order, and effectivity indices of the error estimator in energy norm (Example 2). 


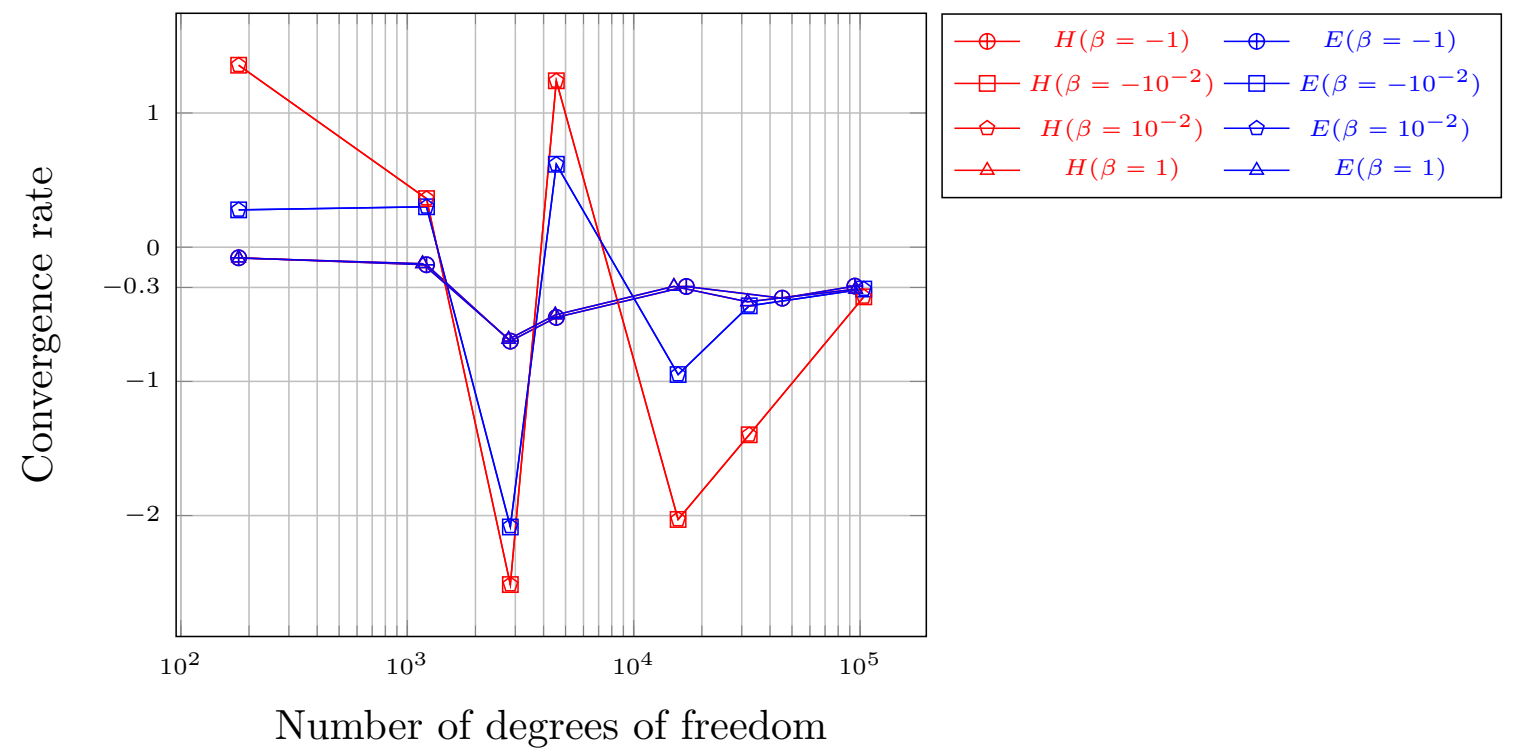

Figure 4.8: Convergence rate of the exact error in $H$-curl norm (red) and energy norm (blue) (Example 2).

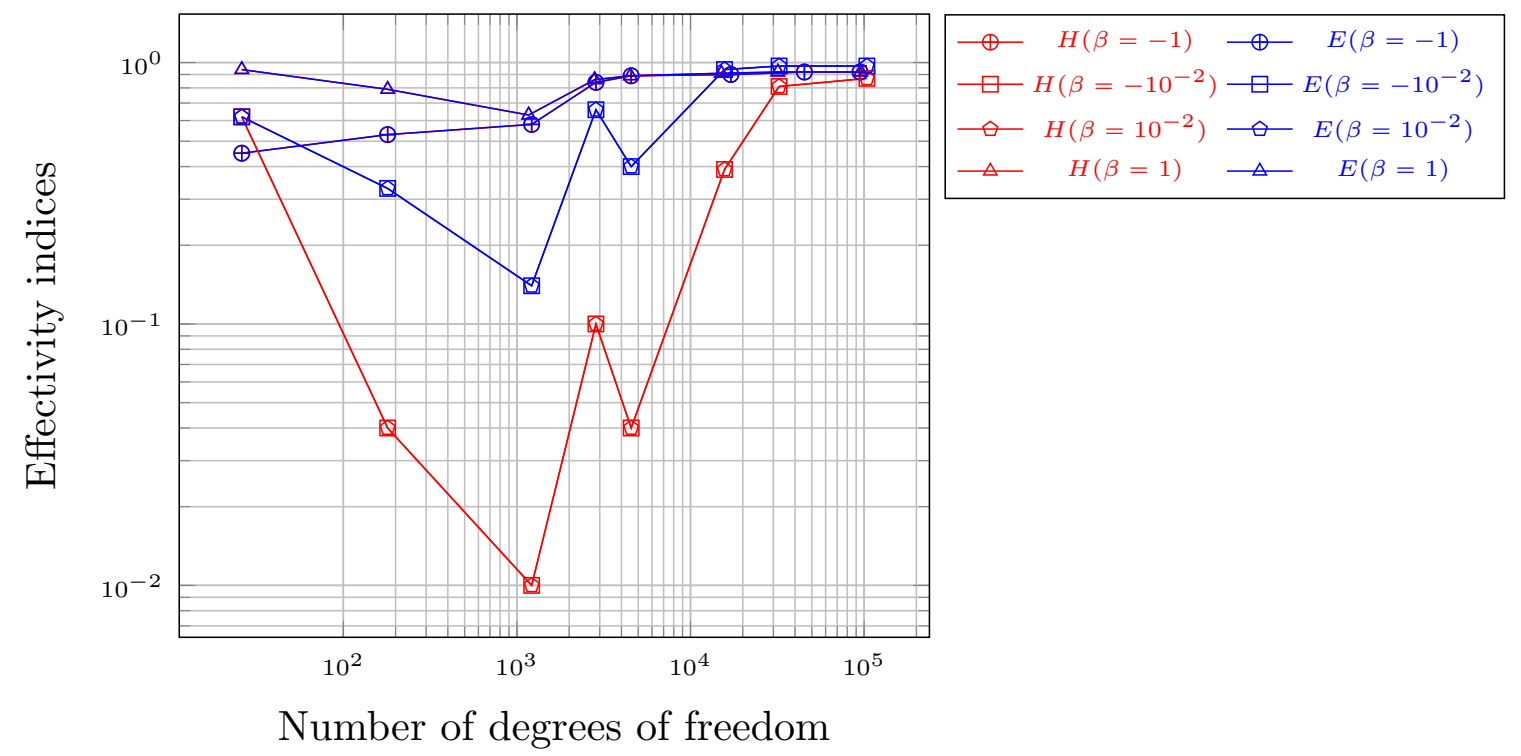

Figure 4.9: Effectivity indices of the error estimators in $H$-curl norm (red) and energy norm (blue) (Example 2). 


\begin{tabular}{|c|c|c|c|}
\hline$\beta$ & $\operatorname{Dof}(V)$ & Cnd. No $(V)$ & Cnd. No $(W)$ \\
\hline \multirow{8}{*}{-1} & 26 & 8.706 & 25.843 \\
\hline & 180 & 72.512 & 67.641 \\
\hline & 1216 & 411.548 & 74.051 \\
\hline & 2852 & 785.997 & 73.619 \\
\hline & 4552 & 1769.605 & 73.886 \\
\hline & 17076 & 3723.828 & 77.157 \\
\hline & 45298 & 4178.156 & 77.203 \\
\hline & 94716 & 4782.625 & 78.790 \\
\hline \multirow{8}{*}{$-10^{-2}$} & 26 & 800.711 & 35.899 \\
\hline & 180 & 4064.624 & 73.977 \\
\hline & 1216 & 18598.227 & 75.668 \\
\hline & 2852 & 46040.994 & 74.531 \\
\hline & 4552 & 59563.815 & 75.142 \\
\hline & 15708 & 124482.043 & 77.293 \\
\hline & 32368 & 202511.238 & 79.686 \\
\hline & 103786 & 387532.191 & 81.221 \\
\hline \multirow{8}{*}{$10^{-2}$} & 26 & 799.289 & 36.252 \\
\hline & 180 & 3584.837 & 74.128 \\
\hline & 1216 & 18565.847 & 75.703 \\
\hline & 2852 & 45948.007 & 74.550 \\
\hline & 4552 & 59373.760 & 75.152 \\
\hline & 15708 & 126551.889 & 77.298 \\
\hline & 32368 & 214002.288 & 79.689 \\
\hline & 103786 & 480710.083 & 81.223 \\
\hline \multirow{8}{*}{1} & 26 & 7.279 & 89.649 \\
\hline & 180 & 35.211 & 83.392 \\
\hline & 1168 & 174.063 & 73.656 \\
\hline & 2804 & 334.503 & 69.854 \\
\hline & 4504 & 340.850 & 75.344 \\
\hline & 15048 & 699.683 & 77.063 \\
\hline & 31852 & 1038.431 & 79.739 \\
\hline & 95494 & 3308.255 & 79.402 \\
\hline
\end{tabular}

Table 4.6: Condition numbers of the stiffness matrices for $V$ and $W$ (Example 2). 


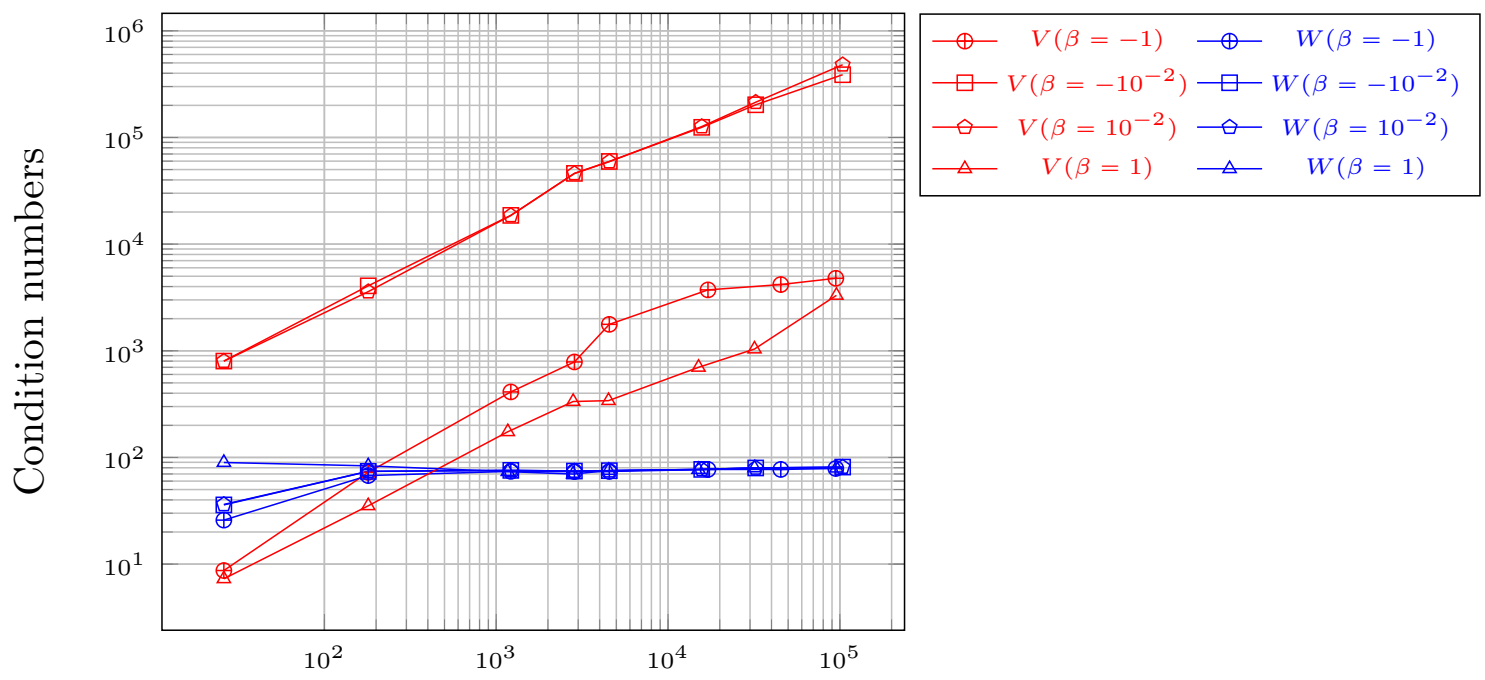

Number of degrees of freedom

Figure 4.10: Condition numbers of the stiffness matrices for $V$ (red) and $W$ (blue) (Example 2).

\subsection{Conclusion}

We have presented, both theoretically and empirically, a hierarchical error estimator, both in terms of estimating global and local errors, for the finite element solution of Maxwells equations in the time-harmonic formulation. The analysis is based on the computation of an approximate error function $\varepsilon \approx \mathbf{u}-\hat{\mathbf{u}}$ in an auxiliary discrete space $W$, where the choice of this auxiliary (error) space is crucial to the success of this approach. We showed that the estimate $\|\varepsilon\|_{H(\operatorname{curl} ; \Omega)}$ of $\|\mathbf{u}-\hat{\mathbf{u}}\|_{H(\operatorname{curl} ; \Omega)}$ is provably reliable and efficient, and the cost of computing $\varepsilon \in W$ is reasonable and much less than that of computing $\hat{\mathbf{u}} \in V$. These theoretical results are clearly observed in the numerical experiments presented in this chapter for the proposed estimator on uniformly/adaptively refined meshes. 


\section{Chapter 5}

\section{The goal-oriented error estimator}

\subsection{Introduction}

It is frequently in finite element analysis that we are more interested in a specic (e.g., localized) feature of the finite element approximation than in the global energy norm. For instance, in some of EM geophysical applications, we focus on the accuracy of the finite element solution at some specific discrete points in the model domain, i.e., the finite element solution only needs to be accurate in some subdomains (where the geophysical observations are made) of the model. In this case, accurate responses at these locations can usually be accomplished with a much sparser optimal mesh using the adaptive refinement approach. While reducing the global error in the finite element solution represented by (4.1) is useful when considering the solution's accuracy over the entire model domain [16], another efficient alternative approach can be found by providing some approximation functional G(u) to measure the accuracy of the solution in some subdomains $\Omega_{s}$ (quantities of interest) of the model. As a result, refinement only occurs where the solution is inaccurate and where such inaccuracy corrupts the solution at a specific quantities. Such methods fall under the category of goal-oriented error estimation $6,15,23,32$. In this study, we are interested in a dual functional $G: H(\operatorname{curl} ; \Omega) \rightarrow \mathbb{C}$ of the error, $G(\mathbf{u}-\hat{\mathbf{u}})$. In other words, the goal is to reduce the error in the quantity of interest, $|G(\mathbf{u}-\hat{\mathbf{u}})|$, rather than the global norm error in $\mathbf{u},\|\mathbf{u}-\hat{\mathbf{u}}\|_{H(\text { curl } ; \Omega)}$.

The key for estimating the error in such quantities is based on the solution of an auxiliary (dual) problem, adjoint to the original (primal) problem. In the following 
we recall the basic framework of this technique.

\subsection{Basic Duality/Adjoint Framework}

We include the basic duality/adjoint framework and our key result of this approach. For more clarification, we start by recalling the problems (2.12), (3.13), and 4.1)

Find $\mathbf{u} \in \mathcal{H}$ such that $B(\mathbf{u}, \mathbf{v})=F(\mathbf{v})$ for all $\mathbf{v} \in \mathcal{H}$,

Find $\hat{\mathbf{u}} \in V$ such that $B(\hat{\mathbf{u}}, \mathbf{v})=F(\mathbf{v})$ for all $\mathbf{v} \in V$,

Find $\varepsilon \in W$ such that $B(\varepsilon, \mathbf{v})=F(\mathbf{v})-B(\hat{\mathbf{u}}, \mathbf{v})$ for all $\mathbf{v} \in W$.

Equation (3.13) is the primal problem; the error in its solution, $\mathbf{u}-\hat{\mathbf{u}} \approx \varepsilon \in W$, is approximated with the residual equation (4.1).

Given a functional $G$, the dual/adjoint problems associated with this functional are as follows:

Find $\mathbf{z} \in \mathcal{H}$ such that $B(\mathbf{v}, \mathbf{z})=G(\mathbf{v})$ for all $\mathbf{v} \in \mathcal{H}$

Find $\hat{\mathbf{z}} \in V$ such that $B(\mathbf{v}, \hat{\mathbf{z}})=G(\mathbf{v})$ for all $\mathbf{v} \in V$,

Find $\delta \in W$ such that $B(\mathbf{v}, \delta)=G(\mathbf{v})-B(\mathbf{v}, \hat{\mathbf{z}})$ for all $\mathbf{v} \in W$.

Equation 5.2 is the discrete dual problem and the error in its solution, $\mathbf{z}-\hat{\mathbf{z}} \approx$ $\delta \in W$, is similarly approximated with the residual equation (5.3). We emphasize the reversal of the roles in the arguments between the dual problem 5.2 and the primal problem (3.13), and on the discrete level, we note that the matrices associated with the computation of (approximate) solutions are conjugate-transposes (adjoints) of each other and based on our assumptions on the model problem, they are identical.

Our objective in the following is to find an estimate for the error

$$
G(\mathbf{u}-\hat{\mathbf{u}})=G(\mathbf{u})-G(\hat{\mathbf{u}})
$$


where $G(\hat{\mathbf{u}})$ denotes the finite element approximation of the quantity of interest and $G(\mathbf{u})$ is the exact value. The primal and dual solutions, $\mathbf{u}$ and $\mathbf{z}$, satisfy the following key relation:

$$
G(\mathbf{u})=B(\mathbf{u}, \mathbf{z})=F(\mathbf{z})
$$

In terms of the approximation error $G(\mathbf{u}-\hat{\mathbf{u}})$, we have

$$
G(\mathbf{u}-\hat{\mathbf{u}})=B(\mathbf{u}-\hat{\mathbf{u}}, \mathbf{z})=F(\mathbf{z})-B(\hat{\mathbf{u}}, \mathbf{z})
$$

In this equation, the middle term shows that the dual solution $\mathbf{z}$ could be thought of as a sensitivity term for the error $\mathbf{u}-\hat{\mathbf{u}}$, which serves to weight such error according to its influence on the dual functional $G(\mathbf{u})$. This idea has been successfully applied in 23, 31, and has proven useful for EM geophysical applications 24, 26, 27, 32, 33.

We further manipulate (5.4) by employing the Galerkin orthogonality condition and primal and dual problems to obtain

$$
\begin{aligned}
G(\mathbf{u}-\hat{\mathbf{u}}) & =B(\mathbf{u}-\hat{\mathbf{u}}, \mathbf{z}) \\
& =B(\mathbf{u}-\hat{\mathbf{u}}, \mathbf{z}-\hat{\mathbf{z}})=B(\mathbf{u}-\hat{\mathbf{u}}-\varepsilon+\varepsilon, \mathbf{z}-\hat{\mathbf{z}}) \\
& =B(\mathbf{u}-\hat{\mathbf{u}}-\varepsilon, \mathbf{z}-\hat{\mathbf{z}})+B(\varepsilon, \mathbf{z}-\hat{\mathbf{z}}) \\
& =B(\mathbf{u}-\hat{\mathbf{u}}-\varepsilon, \mathbf{z}-\hat{\mathbf{z}})+B(\varepsilon, \delta)
\end{aligned}
$$

from problem 4.1, we get

$$
G(\mathbf{u}-\hat{\mathbf{u}})=\underbrace{B(\mathbf{u}-\hat{\mathbf{u}}-\varepsilon, \mathbf{z}-\hat{\mathbf{z}}-\delta)}_{\text {oscillation }}+\underbrace{B(\mathbf{u}-\hat{\mathbf{u}}, \delta)}_{=B(\varepsilon, \delta)},
$$

where $B(\varepsilon, \delta)$ is computable, and the other term will yield a higher-order oscillation. We also define the local element error indicator for element $T$ as:

$$
\eta_{T}=\left|B_{T}(\varepsilon, \delta)\right|
$$


$\eta_{T}$ values are used to decide which elements should be refined to further decrease the error. In practice, the oscillation term vanishes more rapidly than $G(\mathbf{u}-\hat{\mathbf{u}})$ as the mesh is refined, so the error in the goal function is then :

$$
G(\mathbf{u}-\hat{\mathbf{u}}) \approx B(\varepsilon, \delta)
$$

\subsection{Adaptive Refinement Scheme for Numerical Accuracy}

The simplest strategy to control the approximation error consists in an iterative process whose steps are described below.

1. Construct an initial coarse Delaunay triangulation of the model domain $\Omega$.

2. Solve equations (3.13), 4.1), 5.2), and (5.3) and apply the goal-oriented error estimation method as described to produce the local error indicator $\eta_{T}$ for each element.

3. Identify and mark elements with the largest errors for refinement.

4. This process is repeated until the functional error is less than a given preset tolerance.

At first glance, one might think, solving equations (4.1), 5.2), and (5.3) appear to be quite expensive since they require the solution of three additional linear systems. However, note that the primary and dual problems share the same system matrix due to symmetry. In other words, the stiffness matrix that used to solve the primary problem (3.13) is reused for the dual problem (5.2); thus solving the dual problem only requires a new sequence of forward and backward substitutions of the factored matrix. 


\subsection{Numerical Experiments}

In this section, we study the performance of the error estimates with respect to some approximation functionals $G(\mathbf{u})$ in some subdomains $\Omega_{s}$ (quantities of interest) of the model $\Omega$. We now illustrate the theory with examples. In all experiments, we employ adaptive mesh refinements based on the goal-oriented technique, and comparisons are also made with global error estimates in terms of H-curl norms and effectivities.

\subsubsection{Example 1}

In this example, we recall the problem in section 4.6 .2 with values of $\beta$ are $-10^{-2}$, \pm 1 , and \pm 10 . We seek the solution of the equation 2.9 on a unit cube $\Omega=(-1,1)^{3}$ with a homogeneous boundary condition that $\mathbf{u}=0$ on $\partial \Omega$. Here, we consider the particular problem where the exact solution $\mathbf{u}$ is given by the function

$$
\mathbf{u}(x)=\mathbf{u}\left(x_{1}, x_{2}, x_{3}\right)=\left(\begin{array}{l}
1 \\
1 \\
1
\end{array}\right)\left(1-x_{1}^{2}\right)\left(1-x_{2}^{2}\right)\left(1-x_{3}^{2}\right)\left(x_{1}^{2}+x_{2}^{2}+x_{3}^{2}+10^{-2}\right)^{-0.4} .
$$

The load function $f$ can be calculated exactly from $(2.9)$, for the given solution $\mathbf{u}$.

We choose the dual functional $G(\mathbf{u})$, which measures the error in a functional sense, as

$$
G(\mathbf{u})=\int_{\Omega_{s}}(\nabla \times \mathbf{u}) \cdot(\nabla \times \varepsilon) d V .
$$

The subdomain $\Omega_{s}$ is defined as $\Omega_{s}=\Omega_{1} \cup \Omega_{2} \cup \Omega_{3} \cup \Omega_{4}$, where $\left\{\Omega_{i}\right\}_{i=1}^{4}$ are solid cylinders having radius 0.2 and lengths 0.5 and locating on $y$ and $x$ axises as showen in the Figure 5.1. We show in Figure 5.2 a cross sectional view (at $z=0$ ) of intermediate mesh $\left(\operatorname{DoF}(V) N_{o}=121214\right)$ and the final mesh $\left(D o F(V) N_{o}=696351\right)$ for $\beta=1$. We observe that the meshes have been refined in $\Omega_{s}$, and they are much denser close 


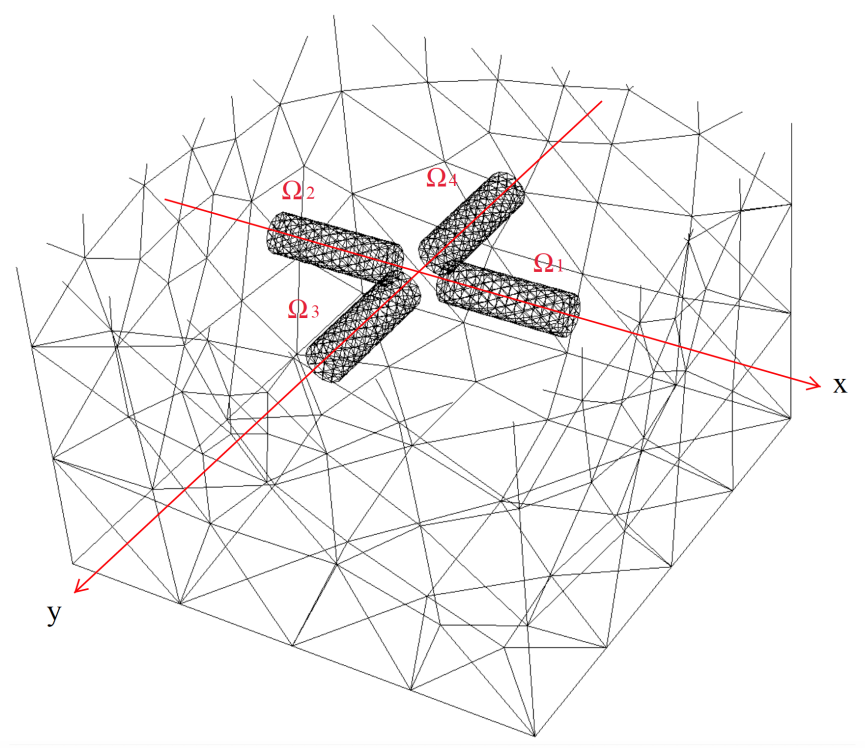

Figure 5.1: The subdomain $\Omega_{s}$ of the domain $\Omega$ (Example 1).

to the origin due to the singularity of $\mathbf{u}$. In Figure 5.3, we show a cross sectional view (at $z=0$ ) of the approximate dual solution for both intermediate and final meshes for $\beta=1$. 

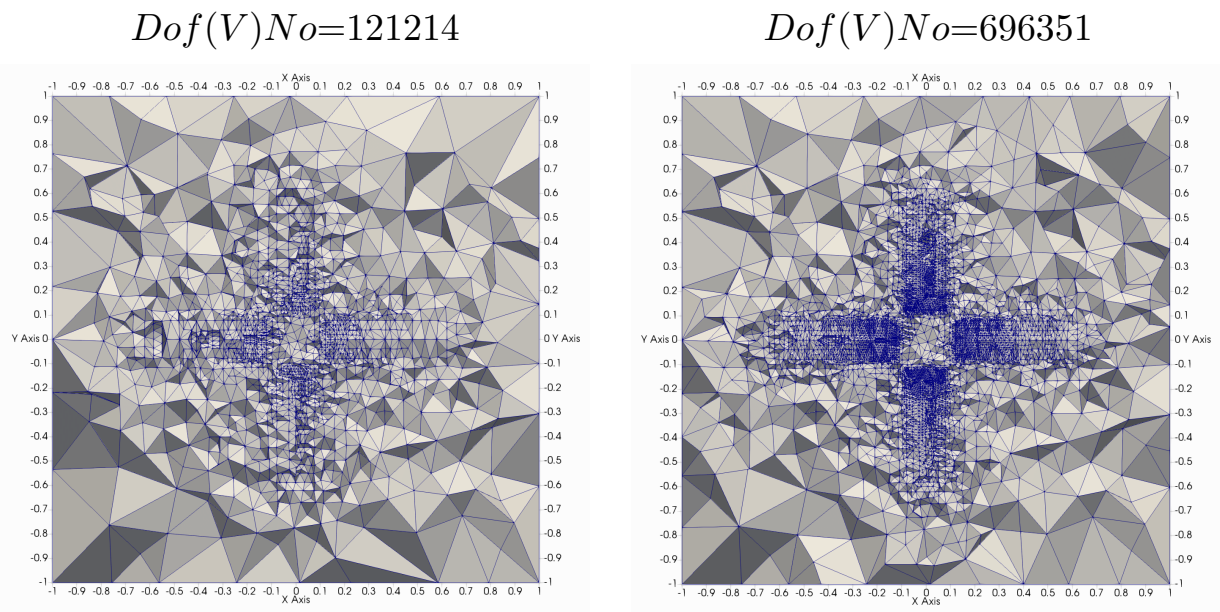

Figure 5.2: Adapted meshes, intermediate and final, to control $G(\mathbf{u})$ in $\Omega_{s}$ when $\beta=1$ (Example 1).
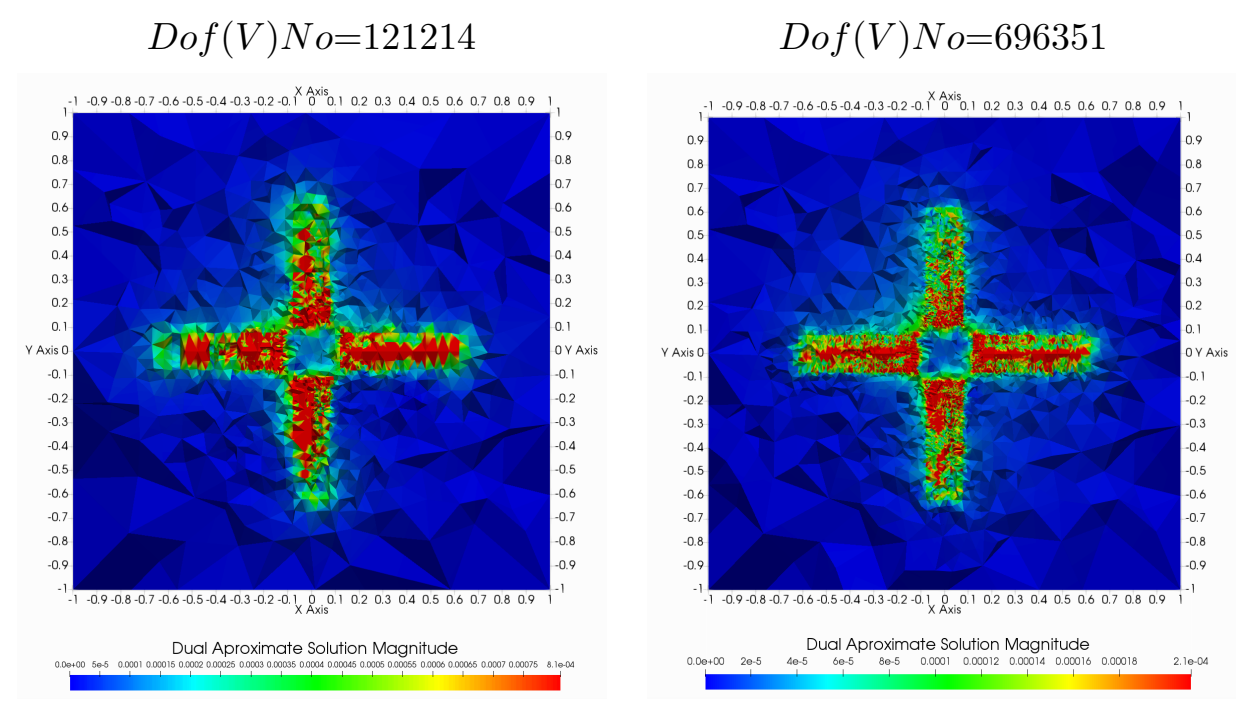

Figure 5.3: Approximate Dual Solutions for intermediate and final meshes for $\beta=1$ (Example 1). 
For comparison purposes, we show goal-oriented error estimations of $B(\varepsilon, \delta)$ and $\|\nabla \times(\mathbf{u}-\hat{\mathbf{u}})\|_{L^{2}\left(\Omega_{s}\right)}^{2}$ in Table 5.1, and error estimators of the global norm errors $\|\varepsilon\|_{H(\operatorname{curl} ; \Omega)}$ and $\|\varepsilon\|$ in Table 5.2. As can be shown in Figure 5.4, the goal-oriented error estimators are smaller than the global error estimators with the same number of degrees of freedom. It is also important to note that as the refinement procedure is continued, the effectivity indices of the estimates computed on a sequence of meshes obtained by goal-oriented refinement are close to one whatever the level of refinement, see Figure 5.5. This indicates that the error obtained from the goal-oriented error estimator is a good approximation of the exact error.

The condition numbers of the diagonally-rescaled stiffness matrices for $W$ and $V$ are showen in Table 5.3. In Figure 5.6, we observe that the computed condition numbers for stiffness matrix for $W$ remained stable and bounded and in a relatively narrow range [113: 141], for all choices of $\beta$. However, the condition numbers for stiffness matrix for $V$ are growing as the mesh is adaptively refined, and their ranges vary based on the difference of $\beta$ values except at $\beta=10$ where they are small with range [20:41]. 


\begin{tabular}{|c|c|c|c|c|c|c|}
\hline$\beta$ & $\operatorname{Dof}(V)$ & $G(\mathbf{u}-\hat{\mathbf{u}})$ & $B(\varepsilon, \delta)$ & $\mathrm{EFF}$ & $\|\nabla \times(\mathbf{u}-\hat{\mathbf{u}})\|_{L^{2}\left(\Omega_{s}\right)}^{2}$ & $\mathrm{EFF}$ \\
\hline \multirow{7}{*}{$-10^{2}$} & 29814 & $4.6176 \mathrm{E}-02$ & $4.8507 \mathrm{E}-02$ & 1.05 & $4.8420 \mathrm{E}-02$ & 1.05 \\
\hline & 47169 & $2.1809 \mathrm{E}-02$ & $2.2743 \mathrm{E}-02$ & 1.04 & 2.3105E-02 & 1.06 \\
\hline & 71178 & $1.3803 \mathrm{E}-02$ & $1.4040 \mathrm{E}-02$ & 1.02 & $1.4820 \mathrm{E}-02$ & 1.07 \\
\hline & 130916 & $6.7803 \mathrm{E}-03$ & $6.8413 \mathrm{E}-03$ & 1.01 & $7.4859 \mathrm{E}-03$ & 1.10 \\
\hline & 217824 & $4.5484 \mathrm{E}-03$ & 4.4712E-03 & 0.98 & $5.1084 \mathrm{E}-03$ & 1.12 \\
\hline & 463638 & $2.2469 \mathrm{E}-03$ & $2.1954 \mathrm{E}-03$ & 0.98 & $2.5750 \mathrm{E}-03$ & 1.15 \\
\hline & 741215 & $1.5215 \mathrm{E}-03$ & $1.4723 \mathrm{E}-03$ & 0.97 & 1.7700E-03 & 1.16 \\
\hline \multirow{7}{*}{-10} & 29814 & $4.5764 \mathrm{E}-02$ & $4.4728 \mathrm{E}-02$ & 0.98 & $4.8342 \mathrm{E}-02$ & 1.06 \\
\hline & 48100 & $2.0804 \mathrm{E}-02$ & 2.0207E-02 & 0.97 & $2.2227 \mathrm{E}-02$ & 1.07 \\
\hline & 62544 & $1.5282 \mathrm{E}-02$ & $1.4830 \mathrm{E}-02$ & 0.97 & 1.6441E-02 & 1.08 \\
\hline & 123556 & $6.8679 \mathrm{E}-03$ & $6.6216 \mathrm{E}-03$ & 0.96 & 7.6129E-03 & 1.11 \\
\hline & 185473 & 4.9973E-03 & 4.8063E-03 & 0.96 & $5.6114 \mathrm{E}-03$ & 1.12 \\
\hline & 402280 & $2.4696 \mathrm{E}-03$ & $2.3647 \mathrm{E}-03$ & 0.96 & $2.8262 \mathrm{E}-03$ & 1.14 \\
\hline & 683309 & $1.6037 \mathrm{E}-03$ & $1.5337 \mathrm{E}-03$ & 0.96 & $1.8589 \mathrm{E}-03$ & 1.16 \\
\hline \multirow{7}{*}{-1} & 29814 & $4.5705 \mathrm{E}-02$ & $4.4580 \mathrm{E}-02$ & 0.98 & $4.8390 \mathrm{E}-02$ & 1.06 \\
\hline & 48108 & $2.0754 \mathrm{E}-02$ & 2.0113E-02 & 0.97 & $2.2234 \mathrm{E}-02$ & 1.07 \\
\hline & 62322 & $1.5338 \mathrm{E}-02$ & $1.4864 \mathrm{E}-02$ & 0.97 & $1.6552 \mathrm{E}-02$ & 1.08 \\
\hline & 120134 & 7.0310E-03 & $6.7722 \mathrm{E}-03$ & 0.96 & 7.8153E-03 & 1.11 \\
\hline & 189784 & $4.9071 \mathrm{E}-03$ & $4.7153 \mathrm{E}-03$ & 0.96 & $5.5311 \mathrm{E}-03$ & 1.13 \\
\hline & 427750 & $2.3496 \mathrm{E}-03$ & $2.2470 \mathrm{E}-03$ & 0.96 & 2.7103E-03 & 1.15 \\
\hline & 674592 & $1.6090 \mathrm{E}-03$ & $1.5380 \mathrm{E}-03$ & 0.96 & $1.8770 \mathrm{E}-03$ & 1.17 \\
\hline \multirow{7}{*}{1} & 29814 & $4.5690 \mathrm{E}-02$ & $4.4555 \mathrm{E}-02$ & 0.98 & $4.8417 \mathrm{E}-02$ & 1.06 \\
\hline & 48108 & $2.0745 \mathrm{E}-02$ & $2.0098 \mathrm{E}-02$ & 0.97 & $2.2252 \mathrm{E}-02$ & 1.07 \\
\hline & 62161 & $1.5391 \mathrm{E}-02$ & $1.4911 \mathrm{E}-02$ & 0.97 & $1.6627 \mathrm{E}-02$ & 1.08 \\
\hline & 121214 & $6.9575 \mathrm{E}-03$ & 6.6970E-03 & 0.96 & 7.7571E-03 & 1.11 \\
\hline & 188339 & $4.9007 \mathrm{E}-03$ & $4.7078 \mathrm{E}-03$ & 0.96 & $5.5382 \mathrm{E}-03$ & 1.13 \\
\hline & 438178 & $2.3018 \mathrm{E}-03$ & $2.2004 \mathrm{E}-03$ & 0.96 & $2.6647 \mathrm{E}-03$ & 1.16 \\
\hline & 696351 & $1.5684 \mathrm{E}-03$ & $1.4989 \mathrm{E}-03$ & 0.96 & $1.8362 \mathrm{E}-03$ & 1.17 \\
\hline \multirow{7}{*}{10} & 29814 & $4.5585 \mathrm{E}-02$ & $4.4330 \mathrm{E}-02$ & 0.97 & 4.8619E-02 & 1.07 \\
\hline & 48093 & $2.0701 \mathrm{E}-02$ & $1.9997 \mathrm{E}-02$ & 0.97 & $2.2417 \mathrm{E}-02$ & 1.08 \\
\hline & 62125 & $1.5377 \mathrm{E}-02$ & $1.4862 \mathrm{E}-02$ & 0.97 & $1.6842 \mathrm{E}-02$ & 1.10 \\
\hline & 123474 & $6.8564 \mathrm{E}-03$ & $6.5794 \mathrm{E}-03$ & 0.96 & 7.8552E-03 & 1.15 \\
\hline & 188042 & $4.9053 \mathrm{E}-03$ & $4.7032 \mathrm{E}-03$ & 0.96 & $5.7077 \mathrm{E}-03$ & 1.16 \\
\hline & 438941 & $2.2812 \mathrm{E}-03$ & $2.1763 \mathrm{E}-03$ & 0.95 & $2.7406 \mathrm{E}-03$ & 1.20 \\
\hline & 707149 & $1.5480 \mathrm{E}-03$ & $1.4764 \mathrm{E}-03$ & 0.95 & $1.8958 \mathrm{E}-03$ & 1.22 \\
\hline
\end{tabular}

Table 5.1: Numerical errors and effectivity indices of goal-oriented error estimator when $\beta \in\left\{-10^{2},-10,-1,1,10\right\}$ (Example 1). 


\begin{tabular}{|c|c|c|c|c|c|c|c|}
\hline$\beta$ & $\operatorname{Dof}(V)$ & $\|\mathbf{u}-\hat{\mathbf{u}}\|_{H(\operatorname{curl} ; \Omega)}$ & $\|\varepsilon\|_{H(\operatorname{curl} ; \Omega)}$ & EFF & $\|\mathbf{u}-\hat{\mathbf{u}}\|$ & $\|\varepsilon\|$ & $\mathrm{EFF}$ \\
\hline \multirow{7}{*}{$-10^{2}$} & 29814 & $1.5531 \mathrm{E}+00$ & $2.0940 \mathrm{E}+00$ & 1.35 & $4.4494 \mathrm{E}+00$ & $2.6293 \mathrm{E}+00$ & 0.59 \\
\hline & 47169 & $1.3983 \mathrm{E}+00$ & $1.8978 \mathrm{E}+00$ & 1.36 & $4.1341 \mathrm{E}+00$ & $2.3668 \mathrm{E}+00$ & 0.57 \\
\hline & 71178 & $1.3272 \mathrm{E}+00$ & $1.8125 \mathrm{E}+00$ & 1.37 & $3.9648 \mathrm{E}+00$ & $2.2330 \mathrm{E}+00$ & 0.56 \\
\hline & 130916 & $1.2346 \mathrm{E}+00$ & $1.6994 \mathrm{E}+00$ & 1.38 & $3.7660 \mathrm{E}+00$ & $2.0605 \mathrm{E}+00$ & 0.55 \\
\hline & 217824 & $1.1368 \mathrm{E}+00$ & $1.5448 \mathrm{E}+00$ & 1.36 & $3.4844 \mathrm{E}+00$ & $1.8169 \mathrm{E}+00$ & 0.52 \\
\hline & 463638 & $1.0869 \mathrm{E}+00$ & $1.4759 \mathrm{E}+00$ & 1.36 & $3.3632 \mathrm{E}+00$ & $1.7294 \mathrm{E}+00$ & 0.51 \\
\hline & 741215 & $1.0698 \mathrm{E}+00$ & $1.4558 \mathrm{E}+00$ & 1.36 & $3.3307 \mathrm{E}+00$ & $1.7074 \mathrm{E}+00$ & 0.51 \\
\hline \multirow{7}{*}{-10} & 29814 & $1.4649 \mathrm{E}+00$ & $1.3813 \mathrm{E}+00$ & 0.94 & $1.9452 \mathrm{E}+00$ & $1.3990 \mathrm{E}+00$ & 0.72 \\
\hline & 48100 & $1.2973 \mathrm{E}+00$ & $1.2145 \mathrm{E}+00$ & 0.94 & $1.7648 \mathrm{E}+00$ & $1.2309 \mathrm{E}+00$ & 0.70 \\
\hline & 62544 & $1.2571 \mathrm{E}+00$ & $1.1751 \mathrm{E}+00$ & 0.93 & $1.7181 \mathrm{E}+00$ & $1.1903 \mathrm{E}+00$ & 0.69 \\
\hline & 123556 & $1.1591 \mathrm{E}+00$ & $1.0797 \mathrm{E}+00$ & 0.93 & $1.6043 \mathrm{E}+00$ & $1.0906 \mathrm{E}+00$ & 0.68 \\
\hline & 185473 & $1.0766 \mathrm{E}+00$ & $9.9976 \mathrm{E}-01$ & 0.93 & $1.4937 \mathrm{E}+00$ & $1.0070 \mathrm{E}+00$ & 0.67 \\
\hline & 402280 & $1.0208 \mathrm{E}+00$ & $9.4532 \mathrm{E}-01$ & 0.93 & $1.4230 \mathrm{E}+00$ & $9.5186 \mathrm{E}-01$ & 0.67 \\
\hline & 683309 & $9.8767 \mathrm{E}-01$ & $9.1259 \mathrm{E}-01$ & 0.92 & $1.3818 \mathrm{E}+00$ & $9.1892 \mathrm{E}-01$ & 0.67 \\
\hline \multirow{7}{*}{-1} & 29814 & $1.4625 \mathrm{E}+00$ & $1.3645 \mathrm{E}+00$ & 0.93 & $1.4625 \mathrm{E}+00$ & $1.3645 \mathrm{E}+00$ & 0.93 \\
\hline & 48108 & $1.2942 \mathrm{E}+00$ & $1.1985 \mathrm{E}+00$ & 0.93 & $1.2942 \mathrm{E}+00$ & $1.1985 \mathrm{E}+00$ & 0.93 \\
\hline & 62322 & $1.2544 \mathrm{E}+00$ & $1.1597 \mathrm{E}+00$ & 0.92 & $1.2544 \mathrm{E}+00$ & $1.1597 \mathrm{E}+00$ & 0.92 \\
\hline & 120134 & $1.1573 \mathrm{E}+00$ & $1.0649 \mathrm{E}+00$ & 0.92 & $1.1573 \mathrm{E}+00$ & $1.0649 \mathrm{E}+00$ & 0.92 \\
\hline & 189784 & $1.0743 \mathrm{E}+00$ & 9.8561E-01 & 0.92 & $1.0743 \mathrm{E}+00$ & $9.8561 \mathrm{E}-01$ & 0.92 \\
\hline & 427750 & $1.0152 \mathrm{E}+00$ & $9.2934 \mathrm{E}-01$ & 0.92 & $1.0152 \mathrm{E}+00$ & $9.2934 \mathrm{E}-01$ & 0.92 \\
\hline & 674592 & $9.9034 \mathrm{E}-01$ & $9.0509 \mathrm{E}-01$ & 0.91 & $9.9034 \mathrm{E}-01$ & $9.0509 \mathrm{E}-01$ & 0.91 \\
\hline \multirow{7}{*}{1} & 29814 & $1.4627 \mathrm{E}+00$ & $1.3696 \mathrm{E}+00$ & 0.94 & $1.4627 \mathrm{E}+00$ & $1.3696 \mathrm{E}+00$ & 0.94 \\
\hline & 48108 & $1.2945 \mathrm{E}+00$ & $1.2029 \mathrm{E}+00$ & 0.93 & $1.2945 \mathrm{E}+00$ & $1.2029 \mathrm{E}+00$ & 0.93 \\
\hline & 62161 & $1.2547 \mathrm{E}+00$ & $1.1639 \mathrm{E}+00$ & 0.93 & $1.2547 \mathrm{E}+00$ & $1.1639 \mathrm{E}+00$ & 0.93 \\
\hline & 121214 & $1.1574 \mathrm{E}+00$ & $1.0678 \mathrm{E}+00$ & 0.92 & $1.1574 \mathrm{E}+00$ & $1.0678 \mathrm{E}+00$ & 0.92 \\
\hline & 188339 & $1.0845 \mathrm{E}+00$ & $9.9768 \mathrm{E}-01$ & 0.92 & $1.0845 \mathrm{E}+00$ & $9.9768 \mathrm{E}-01$ & 0.92 \\
\hline & 438178 & $1.0107 \mathrm{E}+00$ & $9.2661 \mathrm{E}-01$ & 0.92 & $1.0107 \mathrm{E}+00$ & $9.2661 \mathrm{E}-01$ & 0.92 \\
\hline & 696351 & $9.8973 \mathrm{E}-01$ & $9.0610 \mathrm{E}-01$ & 0.92 & $9.8973 \mathrm{E}-01$ & $9.0610 \mathrm{E}-01$ & 0.9 \\
\hline \multirow{7}{*}{10} & 29814 & $1.4797 \mathrm{E}+00$ & $1.4972 \mathrm{E}+00$ & 1.01 & $1.9773 \mathrm{E}+00$ & $1.5451 \mathrm{E}+00$ & 0.78 \\
\hline & 48093 & $1.3090 \mathrm{E}+00$ & $1.3087 \mathrm{E}+00$ & 1.00 & $1.7920 \mathrm{E}+00$ & $1.3463 \mathrm{E}+00$ & 0.75 \\
\hline & 62125 & $1.2701 \mathrm{E}+00$ & $1.2610 \mathrm{E}+00$ & 0.99 & $1.7465 \mathrm{E}+00$ & $1.2953 \mathrm{E}+00$ & 0.74 \\
\hline & 123474 & $1.1698 \mathrm{E}+00$ & $1.1311 \mathrm{E}+00$ & 0.97 & $1.6280 \mathrm{E}+00$ & $1.1504 \mathrm{E}+00$ & 0.71 \\
\hline & 188042 & $1.0976 \mathrm{E}+00$ & $1.0391 \mathrm{E}+00$ & 0.95 & $1.5272 \mathrm{E}+00$ & $1.0508 \mathrm{E}+00$ & 0.69 \\
\hline & 438941 & $1.0344 \mathrm{E}+00$ & $9.7784 \mathrm{E}-01$ & 0.95 & $1.4489 \mathrm{E}+00$ & $9.8802 \mathrm{E}-01$ & 0.68 \\
\hline & 707149 & $9.9748 \mathrm{E}-01$ & $9.4132 \mathrm{E}-01$ & 0.94 & $1.4022 \mathrm{E}+00$ & $9.5071 \mathrm{E}-01$ & 0.68 \\
\hline
\end{tabular}

Table 5.2: Numerical errors and effectivity indices of global error estimator in H-curl and energy norms when $\beta \in\left\{-10^{2},-10,-1,1,10\right\}$ (Example 1). 


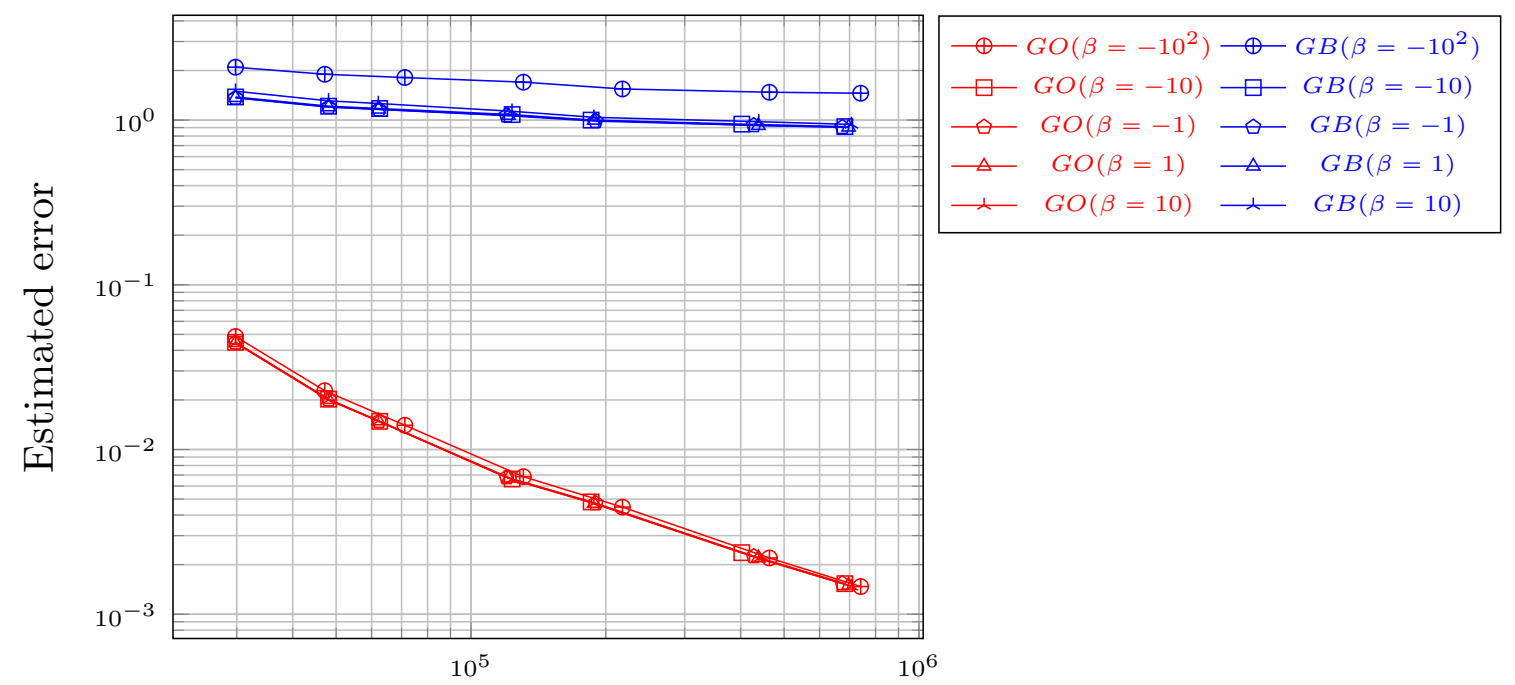

Number of degrees of freedom

Figure 5.4: Error estimators using the global error (GB) and goal-oriented (GO) adaptivity strategy (Example 1).

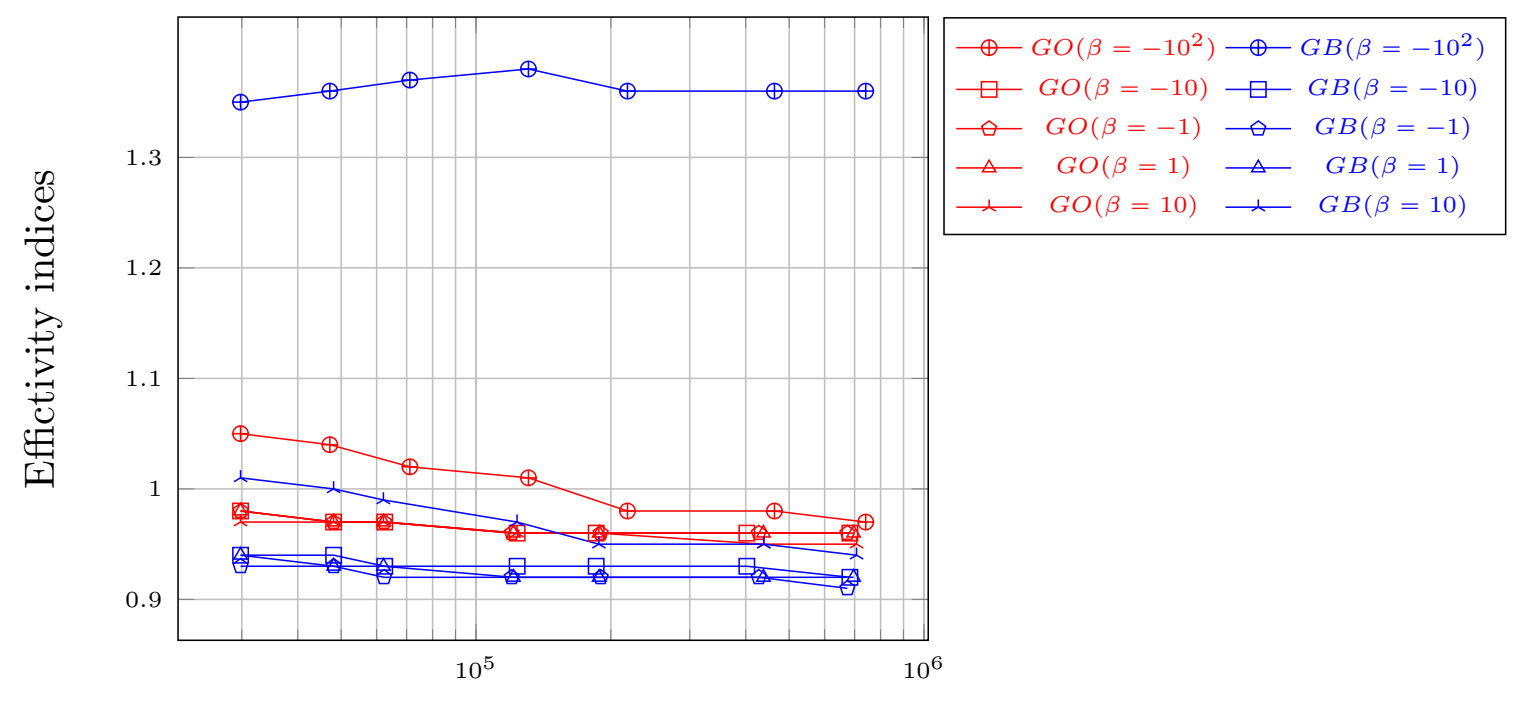

Number of degrees of freedom

Figure 5.5: Effectivity indices using the global error (GB) and goal-oriented (GO) adaptivity strategy (Example 1). 


\begin{tabular}{|c|c|c|c|}
\hline$\beta$ & $\operatorname{Dof}(V)$ & Cnd. No $(V)$ & Cnd. No $(W)$ \\
\hline \multirow{7}{*}{$-10^{2}$} & 29814 & 328.447 & 118.236 \\
\hline & 47169 & 579.807 & 108.057 \\
\hline & 71178 & 828.031 & 109.343 \\
\hline & 130916 & 1485.615 & 117.988 \\
\hline & 217824 & 1790.201 & 124.611 \\
\hline & 463638 & 2896.415 & 132.641 \\
\hline & 741215 & 3691.986 & 141.730 \\
\hline \multirow{7}{*}{-10} & 29814 & 1453.882 & 115.694 \\
\hline & 48100 & 1995.799 & 109.395 \\
\hline & 62544 & 2496.996 & 106.560 \\
\hline & 123556 & 3401.107 & 118.084 \\
\hline & 185473 & 3934.207 & 123.286 \\
\hline & 402280 & 5425.491 & 136.013 \\
\hline & 683309 & 6987.758 & 139.848 \\
\hline \multirow{7}{*}{-1} & 29814 & 5090.769 & 114.360 \\
\hline & 48108 & 6223.700 & 107.838 \\
\hline & 62322 & 7438.939 & 105.961 \\
\hline & 120134 & 8681.565 & 116.942 \\
\hline & 189784 & 10491.221 & 122.438 \\
\hline & 427750 & 13855.012 & 135.747 \\
\hline & 674592 & 16500.720 & 139.288 \\
\hline \multirow{7}{*}{1} & 29814 & 394.594 & 113.944 \\
\hline & 48108 & 527.524 & 107.471 \\
\hline & 62161 & 629.078 & 106.181 \\
\hline & 121214 & 1110.099 & 117.813 \\
\hline & 188339 & 1708.355 & 122.620 \\
\hline & 438178 & 2669.085 & 136.344 \\
\hline & 696351 & 4274.989 & 139.593 \\
\hline \multirow{7}{*}{10} & 29814 & 20.879 & 116.494 \\
\hline & 48093 & 24.495 & 108.961 \\
\hline & 62125 & 26.072 & 108.239 \\
\hline & 123474 & 39.953 & 117.201 \\
\hline & 188042 & 41.642 & 122.311 \\
\hline & 438941 & 41.198 & 136.434 \\
\hline & 707149 & 41.913 & 139.890 \\
\hline
\end{tabular}

Table 5.3: Condition numbers of the stiffness matrices for $V$ and $W$ for $\beta \in$ $\left\{-10^{2},-10,-1,1,10\right\}$ (Example 1). 


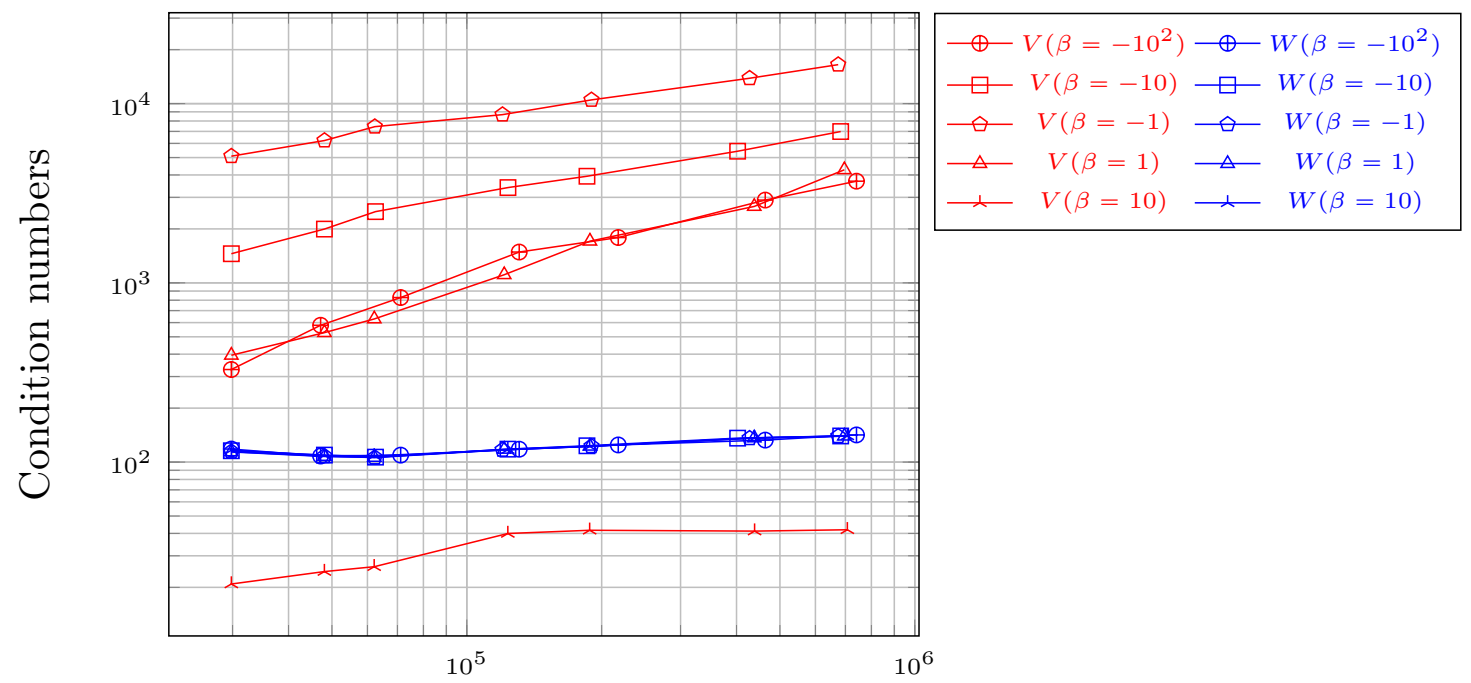

Number of degrees of freedom

Figure 5.6: Condition numbers of the stiffness matrices for $V$ (red) and $W$ (blue) (Example 1).

\subsubsection{Example 2}

Here, In this example, we continue working on the same problem in the previous Example 1 but over different subdomains. As shown in Figure 5.7, the subdomain $\Omega_{s}$ is defined as $\Omega_{s}=\Omega_{1} \cup \Omega_{2} \cup \Omega_{3}$, where $\Omega_{1}$ is a cube centered at $(0.3,0.3,0.3), \Omega_{2}$ is a semi-cube centered at $(-0.3,-0.3,0.3)$, and $\Omega_{3}$ is a sphere centered at $(0,0,-0.4)$ and has radios 0.3. The coefficient $\beta$ is kept constant (equal 1) all over the domain and the linear functional $G(\mathbf{u})$ is defined as in (5.6). In this experiment, we employ goal-oriented error estimates over each sudomain separately then over all subdomains $\Omega_{s}$. The adapted final meshes are showen in Figure 5.8. We observe that the meshes have been refined in quantities of interest $\Omega_{1}, \Omega_{2}, \Omega_{3}$, and $\Omega_{s}$, and they are much denser close to the origin due to the singularity of $\mathbf{u}$. 


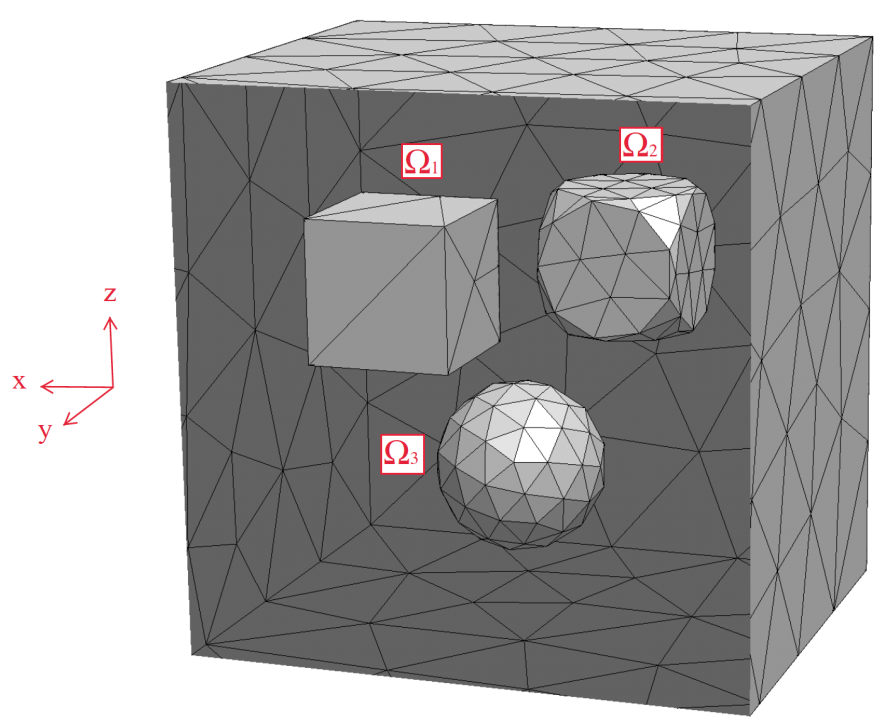

Figure 5.7: The subdomain $\Omega_{s}$ of the domain $\Omega$ (Example 2).

In Tables 5.4. we show the goal oriented error estimators and the global error estimator in the H-curl norm with respect to the number of degrees of freedom on sequences of meshes. As showen in Figure 5.9, the goal oriented error estimators decrease faster than the global error estimators as the mesh refined. Also we see, in Figure 5.10, that the effectivity indices of the two estimates are close to one and that the goal oriented strategy provides better results than the global error estimates in all subdomain cases. 
Final refinement on $\Omega_{1}$

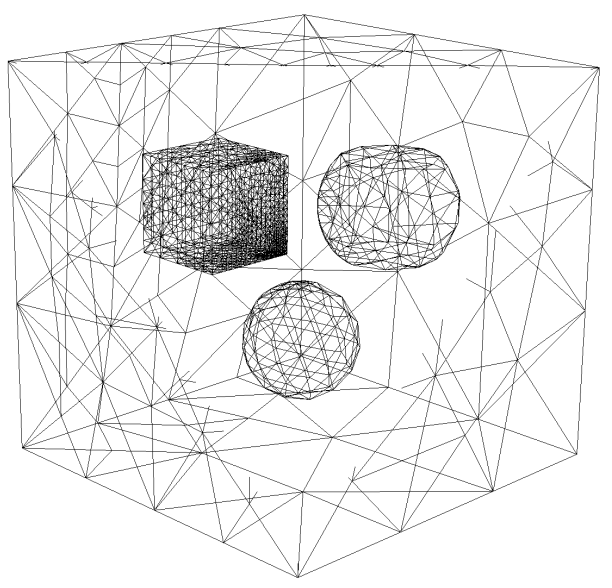

Final refinement on $\Omega_{3}$

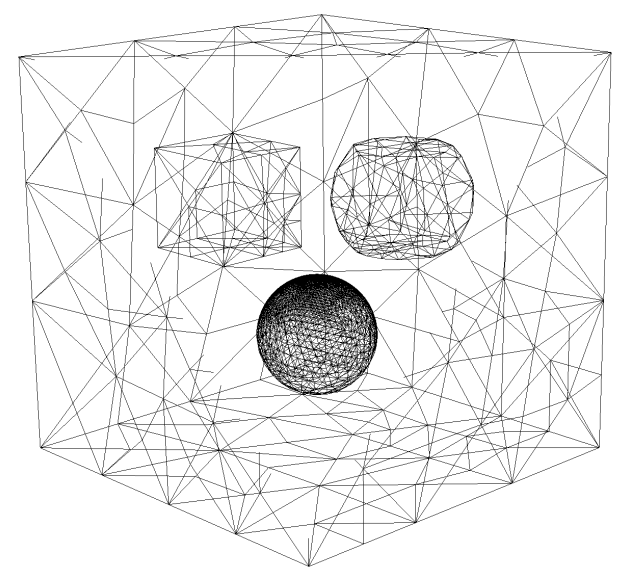

Final refinement on $\Omega_{2}$

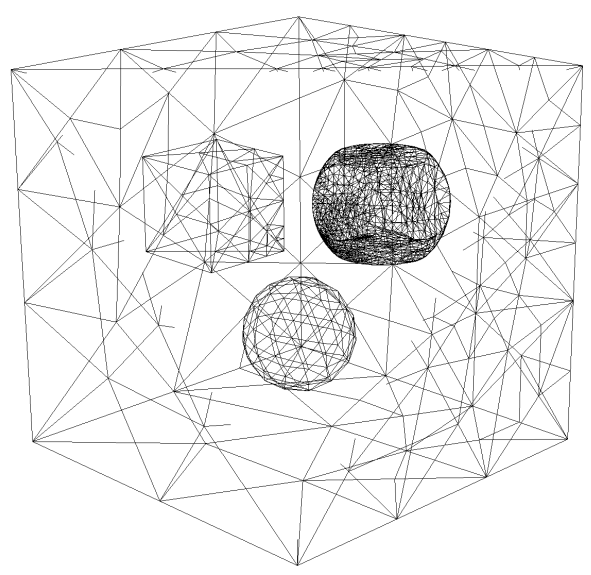

Final refinement on $\Omega_{s}$

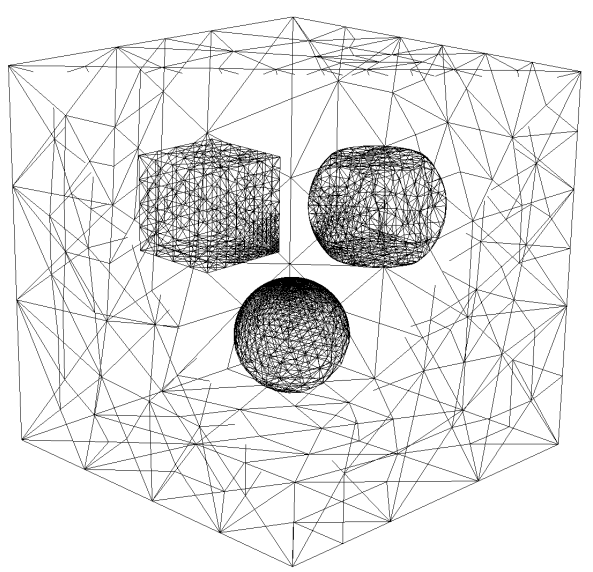

Figure 5.8: The final mesh refinement over each subdomain separately and over all subdomains (Example 2). 


\begin{tabular}{|c|c|c|c|c|c|c|c|}
\hline & & \multicolumn{3}{|c|}{ Goal Oriented Estimates } & \multicolumn{3}{|c|}{ Global Estimates } \\
\hline$\Omega_{i}$ & $\operatorname{Dof}(V)$ & $G(\mathbf{u}-\hat{\mathbf{u}})$ & $B(\varepsilon, \delta)$ & $\mathrm{EFF}$ & $\|\mathbf{u}-\hat{\mathbf{u}}\|_{H(\operatorname{curl} ; \Omega)}$ & $\|\varepsilon\|_{H(\operatorname{curl} ; \Omega)}$ & $\mathrm{EFF}$ \\
\hline \multirow{7}{*}{$\Omega_{1}$} & 2849 & $2.5061 \mathrm{E}-01$ & $2.6250 \mathrm{E}-01$ & 1.05 & $3.3860 \mathrm{E}+00$ & $2.7932 \mathrm{E}+00$ & 0.82 \\
\hline & 3685 & $1.2971 \mathrm{E}-01$ & $1.2727 \mathrm{E}-01$ & 0.98 & $3.1688 \mathrm{E}+00$ & $2.6020 \mathrm{E}+00$ & 0.82 \\
\hline & 4878 & $7.5016 \mathrm{E}-02$ & 7.3192E-02 & 0.98 & $2.7981 \mathrm{E}+00$ & $2.3602 \mathrm{E}+00$ & 0.84 \\
\hline & 8945 & $3.1924 \mathrm{E}-02$ & 3.0799E-02 & 0.96 & $2.4348 \mathrm{E}+00$ & $2.1230 \mathrm{E}+00$ & 0.87 \\
\hline & 17120 & $1.5809 \mathrm{E}-02$ & $1.4996 \mathrm{E}-02$ & 0.95 & $2.2409 \mathrm{E}+00$ & $1.9063 \mathrm{E}+00$ & 0.85 \\
\hline & 30812 & $8.3470 \mathrm{E}-03$ & 7.9173E-03 & 0.95 & $2.1471 \mathrm{E}+00$ & $1.8140 \mathrm{E}+00$ & 0.84 \\
\hline & 62055 & $4.5057 \mathrm{E}-03$ & $4.2391 \mathrm{E}-03$ & 0.94 & $2.1055 \mathrm{E}+00$ & $1.7710 \mathrm{E}+00$ & 0.84 \\
\hline \multirow{7}{*}{$\Omega_{2}$} & 2849 & $2.2522 \mathrm{E}-01$ & $2.2012 \mathrm{E}-01$ & 0.98 & $3.3860 \mathrm{E}+00$ & $2.7932 \mathrm{E}+00$ & 0.82 \\
\hline & 4040 & $9.7817 \mathrm{E}-02$ & $9.3420 \mathrm{E}-02$ & 0.96 & $2.8417 \mathrm{E}+00$ & $2.4028 \mathrm{E}+00$ & 0.85 \\
\hline & 10038 & $3.5929 \mathrm{E}-02$ & $3.3735 \mathrm{E}-02$ & 0.94 & $2.6415 \mathrm{E}+00$ & $2.1812 \mathrm{E}+00$ & 0.83 \\
\hline & 17252 & $1.9974 \mathrm{E}-02$ & $1.8801 \mathrm{E}-02$ & 0.94 & $2.5910 \mathrm{E}+00$ & $2.1266 \mathrm{E}+00$ & 0.82 \\
\hline & 30870 & $1.1161 \mathrm{E}-02$ & $1.0702 \mathrm{E}-02$ & 0.96 & $2.4229 \mathrm{E}+00$ & $1.9745 \mathrm{E}+00$ & 0.81 \\
\hline & 40328 & $9.0806 \mathrm{E}-03$ & $8.6803 \mathrm{E}-03$ & 0.96 & $2.3876 \mathrm{E}+00$ & $1.9359 \mathrm{E}+00$ & 0.81 \\
\hline & 70381 & $5.7861 \mathrm{E}-03$ & $5.5319 \mathrm{E}-03$ & 0.96 & $2.3362 \mathrm{E}+00$ & $1.8846 \mathrm{E}+00$ & 0.81 \\
\hline \multirow{7}{*}{$\Omega_{3}$} & 2849 & 7.0849E-01 & 6.1914E-01 & 0.87 & $3.3860 \mathrm{E}+00$ & $2.7932 \mathrm{E}+00$ & 0.82 \\
\hline & 4613 & $3.9256 \mathrm{E}-01$ & $3.5816 \mathrm{E}-01$ & 0.91 & $2.5854 \mathrm{E}+00$ & $2.2115 \mathrm{E}+00$ & 0.86 \\
\hline & 9141 & $1.6050 \mathrm{E}-01$ & $1.4790 \mathrm{E}-01$ & 0.92 & $2.3826 \mathrm{E}+00$ & $1.9676 \mathrm{E}+00$ & 0.83 \\
\hline & 21998 & $6.8684 \mathrm{E}-02$ & $6.3640 \mathrm{E}-02$ & 0.93 & $2.1611 \mathrm{E}+00$ & $1.7713 \mathrm{E}+00$ & 0.82 \\
\hline & 39955 & $4.1786 \mathrm{E}-02$ & $3.8448 \mathrm{E}-02$ & 0.92 & $2.1308 \mathrm{E}+00$ & $1.7258 \mathrm{E}+00$ & 0.81 \\
\hline & 89236 & $2.0759 \mathrm{E}-02$ & $1.9145 \mathrm{E}-02$ & 0.92 & $2.1189 \mathrm{E}+00$ & $1.7004 \mathrm{E}+00$ & 0.80 \\
\hline & 159169 & $1.3226 \mathrm{E}-02$ & $1.2228 \mathrm{E}-02$ & 0.92 & $2.0524 \mathrm{E}+00$ & $1.6099 \mathrm{E}+00$ & 0.78 \\
\hline \multirow{7}{*}{$\Omega_{s}$} & 2849 & $1.1843 \mathrm{E}+00$ & $1.1018 \mathrm{E}+00$ & 0.93 & $3.3860 \mathrm{E}+00$ & $2.7932 \mathrm{E}+00$ & 0.82 \\
\hline & 4997 & $6.7204 \mathrm{E}-01$ & $6.3261 \mathrm{E}-01$ & 0.94 & $2.5198 \mathrm{E}+00$ & $2.1555 \mathrm{E}+00$ & 0.86 \\
\hline & 10060 & $3.3196 \mathrm{E}-01$ & $3.1405 \mathrm{E}-01$ & 0.95 & $2.2018 \mathrm{E}+00$ & $1.8467 \mathrm{E}+00$ & 0.84 \\
\hline & 21197 & $1.8960 \mathrm{E}-01$ & $1.7809 \mathrm{E}-01$ & 0.94 & $1.9828 \mathrm{E}+00$ & $1.5840 \mathrm{E}+00$ & 0.80 \\
\hline & 45562 & $9.9880 \mathrm{E}-02$ & $9.3950 \mathrm{E}-02$ & 0.94 & $1.8614 \mathrm{E}+00$ & $1.4281 \mathrm{E}+00$ & 0.77 \\
\hline & 78581 & 6.3071E-02 & $5.9275 \mathrm{E}-02$ & 0.94 & $1.8016 \mathrm{E}+00$ & $1.3337 \mathrm{E}+00$ & 0.74 \\
\hline & 151234 & $3.7354 \mathrm{E}-02$ & $3.5165 \mathrm{E}-02$ & 0.94 & $1.7398 \mathrm{E}+00$ & $1.2499 \mathrm{E}+00$ & 0.72 \\
\hline
\end{tabular}

Table 5.4: Numerical errors and effectivity indices of goal-oriented and global estimates over $\Omega_{1}, \Omega_{2}, \Omega_{3}$, and $\Omega_{s}$ (Example 2 ).

In Table 5.5, we present the condition numbers of the diagonally-rescaled stiffness matrices for $W$ and $V$. We notice that the computed condition numbers for stiffness matrix for $W$ decrease in stable and bounded ranges [292:154] on subdomains $\Omega_{1}$ and $\Omega_{2}$, and ranges [292:240] on $\Omega_{1}$ and $\Omega_{2}$. However, the condition numbers for stiffness matrix for $V$ grows as the mesh is adaptively refined, see Figure 5.11 . 


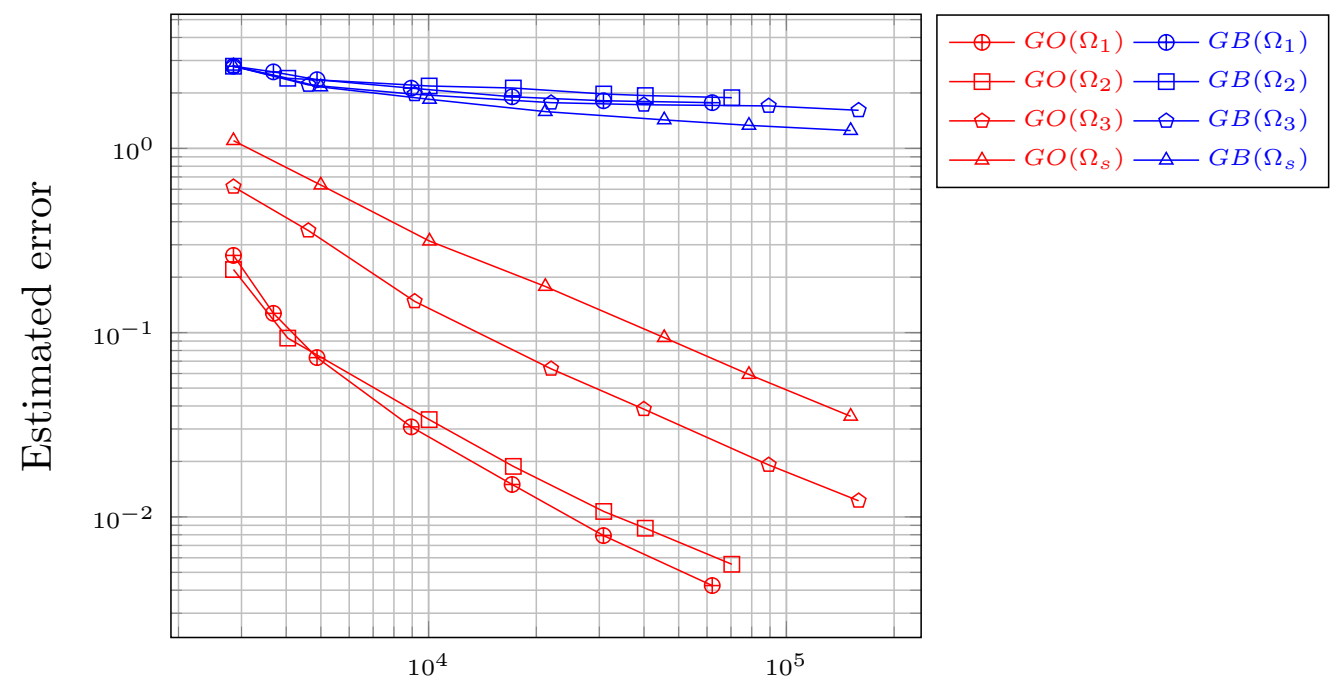

Number of degrees of freedom

Figure 5.9: Error estimators using the global error (GB) and goal-oriented (GO) adaptivity strategy (Example 2).

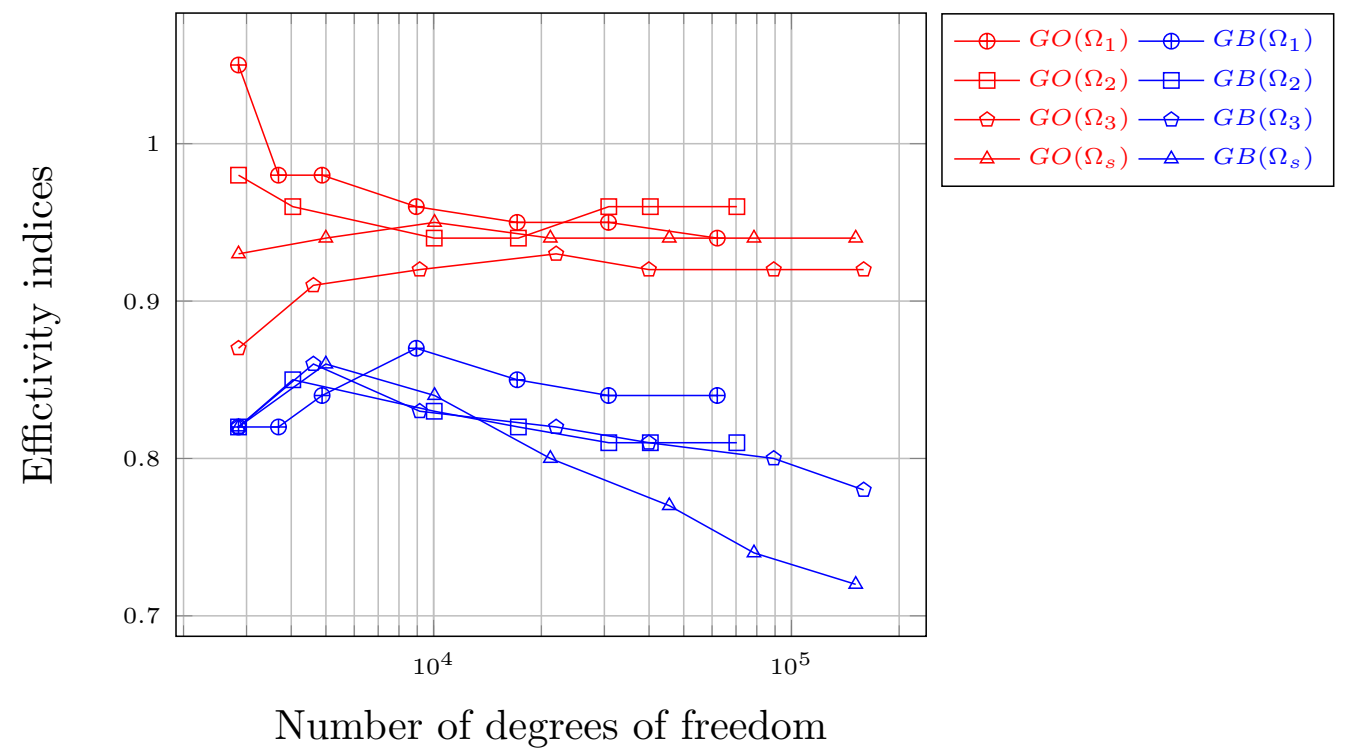

Figure 5.10: Effectivity indices using the global error (GB) and goal-oriented (GO) adaptivity strategy (Example 2). 


\begin{tabular}{l||l|l|l}
\hline$\Omega_{i}$ & Dof $(V)$ & Cnd. No $(V)$ & Cnd. No $(W)$ \\
\hline \multirow{5}{*}{$\Omega_{1}$} & 2849 & 619.713 & 291.975 \\
& 3685 & 739.514 & 250.924 \\
& 4878 & 1036.078 & 214.677 \\
& 8945 & 1681.551 & 177.772 \\
& 17120 & 2680.487 & 155.136 \\
& 30812 & 3777.240 & 163.511 \\
\hline \multirow{5}{*}{$\Omega_{2}$} & 62055 & 5588.615 & 154.055 \\
\hline & 2849 & 619.713 & 291.975 \\
& 4040 & 855.694 & 255.045 \\
& 10038 & 2543.936 & 228.477 \\
& 17252 & 3517.871 & 224.574 \\
& 30870 & 5065.558 & 182.858 \\
& 40328 & 5192.088 & 173.751 \\
& 70381 & 7944.255 & 166.645 \\
\hline \multirow{5}{*}{$\Omega_{3}$} & 2849 & 619.713 & 291.975 \\
& 4613 & 1145.222 & 198.840 \\
& 9141 & 3642.920 & 228.073 \\
& 21998 & 5010.010 & 246.103 \\
& 39955 & 7903.599 & 256.574 \\
& 89236 & 43173.853 & 265.042 \\
& 159169 & 943219.106 & 270.802 \\
\hline \multirow{5}{*}{} & 2849 & 619.713 & 291.975 \\
& 4997 & 1162.885 & 191.543 \\
& 10060 & 2304.632 & 212.929 \\
\hline
\end{tabular}

Table 5.5: Condition numbers of the stiffness matrices for $V$ and $W$ in each choice of subdomains (Example 2). 


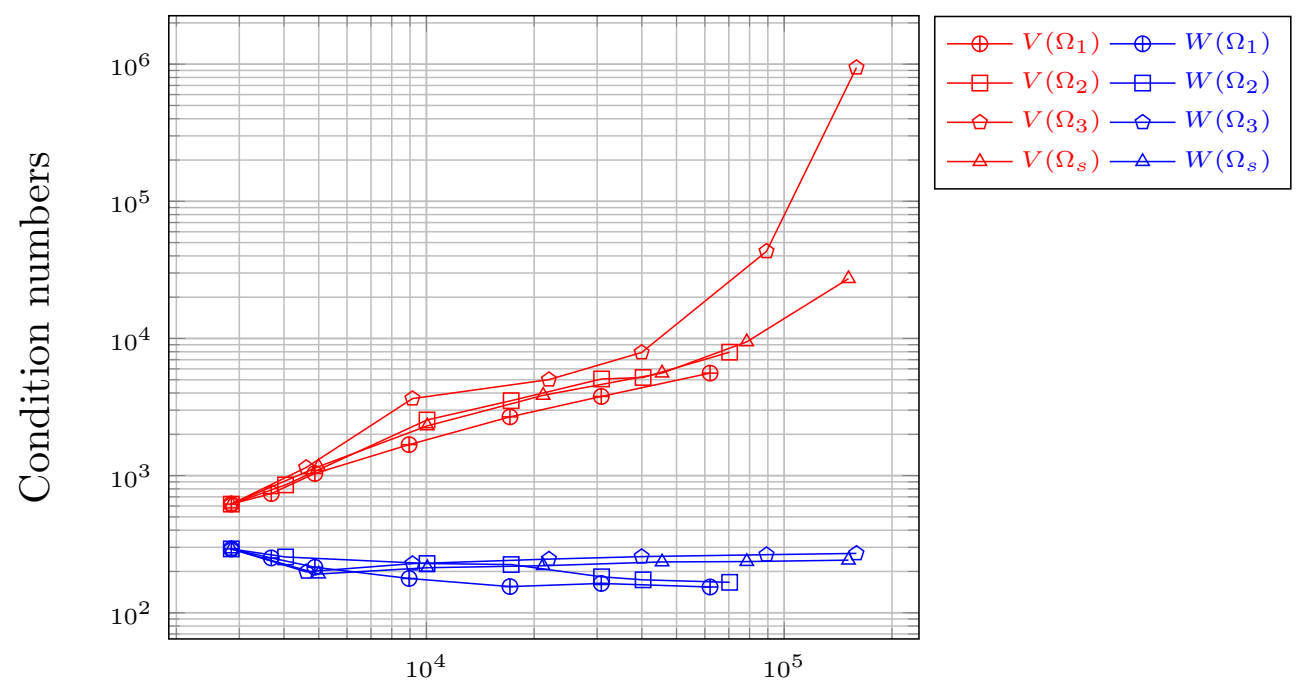

Number of degrees of freedom

Figure 5.11: Condition numbers of the stiffness matrices for $V$ (red) and $W$ (blue) (Example 2).

\subsection{Conclusion}

In this chapter, we presented a new approach in a posteriori error estimation, in which the numerical error of finite element approximations is estimated in terms of quantities of interest rather than the classical global $H$-curl or energy norm. These so-called quantities of interest are characterized by linear functionals on the space of functions to where the solution belongs. Goal-oriented error estimation is based on the notion of the adjoint/dual problem, which is "straightforwardly" derived from the weak formulation of the primal problem. Error estimates and refinement indicators are obtained by solving for approximations to the adjoint problem. The numerical results illustrated that the error in the goal functional is estimated very precisely and can be reduced rapidly by applying the adaptive algorithm. 


\section{REFERENCES}

[1] M. Ainsworth and J. Oden. A posteriori error estimation in finite element analysis. Computer Methods in Applied Mechanics and Engineering, 142(1):1 - 88, 1997.

[2] M. Ainsworth and J. T. Oden. A posteriori error estimation in finite element analysis. Pure and Applied Mathematics (New York). Wiley-Interscience [John Wiley \& Sons], New York, 2000.

[3] R. Bank and R. Smith. A posteriori error estimates based on hierarchical bases. SIAM Journal on Numerical Analysis, 30(4):921-935, 1993.

[4] R. E. Bank. Hierarchical bases and the finite element method. In Acta numerica, 1996, volume 5 of Acta Numer., pages 1-43. Cambridge Univ. Press, Cambridge, 1996.

[5] R. Beck, R. Hiptmair, and B. Wohlmuth. Hierarchical error estimator for eddy current computation. In Numerical mathematics and advanced applications (Jyväskylä, 1999), pages 110-120. World Sci. Publ., River Edge, NJ, 2000.

[6] R. Becker and R. Rannacher. An optimal control approach to a posteriori error estimation in finite element methods. Acta Numerica, 10:1102, 2001.

[7] M. M. Botha and D. B. Davidson. An explicit a posteriori error indicator for electromagnetic, finite element-boundary integral analysis. IEEE Trans. Antennas and Propagation, 53(11):3717-3725, 2005. 
[8] D. Braess and J. Schöberl. Equilibrated residual error estimator for edge elements. Math. Comp., 77(262):651-672, 2008.

[9] A. Buffa and P. Ciarlet, Jr. On traces for functional spaces related to Maxwell's equations. I. An integration by parts formula in Lipschitz polyhedra. Math. Methods Appl. Sci., 24(1):9-30, 2001.

[10] A. Buffa, M. Costabel, and D. Sheen. On traces for $\mathbf{H}(\mathbf{c u r l}, \Omega)$ in Lipschitz domains. J. Math. Anal. Appl., 276(2):845-867, 2002.

[11] M. Bürg. A residual-based a posteriori error estimator for the $h p$-finite element method for Maxwell's equations. Appl. Numer. Math., 62(8):922-940, 2012.

[12] P. G. Ciarlet. The finite element method for elliptic problems. North-Holland Publishing Co., Amsterdam-New York-Oxford, 1978. Studies in Mathematics and its Applications, Vol. 4.

[13] A. W. Craig, J. Z. Zhu, and O. C. Zienkiewicz. A posteriori error estimation, adaptive mesh refinement and multigrid methods using hierarchical finite element bases. In The mathematics of finite elements and applications, $V$ (Uxbridge, 1984), pages 587-594. Academic Press, London, 1985.

[14] P. Deuflhard, P. Leinen, and H. Yserentant. Concepts of an adaptive hierarchical finite element code. IMPACT of Computing in Science and Engineering, 1(1):3 $-35,1989$.

[15] D. Estep, M. Holst, and M. Larson. Generalized Green's functions and the effective domain of influence. SIAM J. Sci. Comput., 26(4):1314-1339, 2005.

[16] A. Franke, R.-U. Börner, and K. Spitzer. Adaptive unstructured grid finite 
element simulation of two-dimensional magnetotelluric fields for arbitrary surface and seafloor topography. Geophysical Journal International, 171(1):71-86, 2007.

[17] J. Gopalakrishnan, L. E. García-Castillo, and L. F. Demkowicz. Nédélec spaces in affine coordinates. Comput. Math. Appl., 49(7-8):1285-1294, 2005.

[18] H. Hakula, M. Neilan, and J. S. Ovall. A posteriori estimates using auxiliary subspace techniques. J. Sci. Comput., 72(1):97-127, 2017.

[19] D. Harutyunyan, F. Izsák, J. J. W. van der Vegt, and M. A. Botchev. Adaptive finite element techniques for the Maxwell equations using implicit a posteriori error estimates. Comput. Methods Appl. Mech. Engrg., 197(17-18):1620-1638, 2008.

[20] M. Holst, J. S. Ovall, and R. Szypowski. An efficient, reliable and robust error estimator for elliptic problems in $\mathbb{R}^{3}$. Applied Numerical Mathematics, 61(5):675 $-695,2011$.

[21] P. Houston, I. Perugia, and D. Schötzau. Energy norm a posteriori error estimation for mixed discontinuous Galerkin approximations of the Maxwell operator. Comput. Methods Appl. Mech. Engrg., 194(2-5):499-510, 2005.

[22] F. Izsák, D. Harutyunyan, and J. J. W. van der Vegt. Implicit a posteriori error estimates for the Maxwell equations. Math. Comp., 77(263):1355-1386, 2008.

[23] K. Key and J. Ovall. A parallel goal-oriented adaptive finite element method for 2.5-d electromagnetic modelling. Geophysical Journal International, 186(1):137$154,2011$.

[24] K. Key and C. Weiss. Adaptive finite-element modeling using unstructured grids: The 2d magnetotelluric example. GEOPHYSICS, 71(6):G291-G299, 2006. 
[25] J. Li and Y. Lin. A priori and posteriori error analysis for time-dependent Maxwell's equations. Comput. Methods Appl. Mech. Engrg., 292:54-68, 2015.

[26] Y. Li and K. Key. 2d marine controlled-source electromagnetic modeling: Part 1 an adaptive finite-element algorithm. GEOPHYSICS, 72(2):WA51-WA62, 2007.

[27] Y. Li and J. Pek. Adaptive finite element modelling of two-dimensional magnetotelluric fields in general anisotropic media. Geophysical Journal International, 175(3):942-954, 122008.

[28] P. Monk. Finite element methods for Maxwell's equations. Numerical Mathematics and Scientific Computation. Oxford University Press, New York, 2003.

[29] J. C. Nedelec. Mixed finite elements in 3. Numerische Mathematik, 35(3):315341, Sep 1980.

[30] S. Nicaise and E. Creusé. A posteriori error estimation for the heterogeneous Maxwell equations on isotropic and anisotropic meshes. Calcolo, 40(4):249-271, 2003.

[31] J. S. Ovall. Duality-based adaptive refinement for elliptic PDEs. ProQuest LLC, Ann Arbor, MI, 2004. Thesis (Ph.D.)-University of California, San Diego.

[32] J. S. Ovall. Asymptotically exact functional error estimators based on superconvergent gradient recovery. Numer. Math., 102(3):543-558, 2006.

[33] Z. Ren, T. Kalscheuer, S. Greenhalgh, and H. Maurer. A goal-oriented adaptive finite-element approach for plane wave 3-D electromagnetic modelling. Geophysical Journal International, 194(2):700-718, 052013. 
[34] J. Schöberl. A posteriori error estimates for Maxwell equations. Math. Comp., $77(262): 633-649,2008$.

[35] J. P. Webb. Hierarchal vector basis functions of arbitrary order for triangular and tetrahedral finite elements. IEEE Trans. Antennas and Propagation, 47(8):1244$1253,1999$.

[36] L. Zhong, S. Shu, G. Wittum, and J. Xu. Optimal error estimates for nedelec edge elements for time-harmonic maxwell's equations. Journal of Computational Mathematics, 27(5):563-572, 2009.

[37] O. C. Zienkiewicz, D. W. Kelly, J. Gago, and I. Babuška. Hierarchical finite element approaches, error estimates and adaptive refinement. In The mathematics of finite elements and applications, IV (Uxbridge, 1981), pages 313-346. Academic Press, London-New York, 1982. 


\section{APPENDIX}

\section{A.1 Additional Numerical Results}

In this appendix, and as a matter of interest, we report further numerical results for the examples given in Section 4.6. In first section, we will report errors and effectivities in the global $L^{2}$ norm for examples 4.6.1 and 4.6.2. In the second section, we will resolve the problems in examples 4.6.1 and 4.6.2 by using first-order Nédélec space of the second-kind and then we report errors and effectivities in the global $H$-curl norm, energy norm, and $L^{2}$ norm.

\section{A.2 Numerical Results in $L^{2}$ norm}

Although there are no (available) prior theoretical results had been done in $L^{2}$ norm in our approach area, we show numerical errors and effectivity indices of the error estimator in $L^{2}$ norm for examples 4.6.1 and 4.6.2.

\section{A.2.1 Example 1}

In Table 6, we show numerical errors and effectivity indices of the error estimator in $L^{2}$ norm for example 4.6.1. It can be observed, for all $\beta$ values, that the convergence

rate of $\|\mathbf{u}-\hat{\mathbf{u}}\|_{L^{2}(\Omega)}$ is optimal, i.e. $O(h)$, whereas the one of $\|\varepsilon\|_{L^{2}(\Omega)}$ is $O\left(h^{2}\right)$. And as result of this rates difference, we have poor effectivity indices. 


\begin{tabular}{|c|c|c|c|c|c|c|}
\hline$\beta$ & $\operatorname{Dof}(V)$ & $\|\mathbf{u}-\hat{\mathbf{u}}\|_{L^{2}(\Omega)}$ & order & $\|\varepsilon\|_{L^{2}(\Omega)}$ & order & $\mathrm{EFF}$ \\
\hline \multirow{6}{*}{-1} & 26 & $1.5337 \mathrm{E}+00$ & & $2.6555 \mathrm{E}-01$ & & 0.17 \\
\hline & 180 & $9.6301 \mathrm{E}-01$ & 0.671 & $3.4191 \mathrm{E}-01$ & -0.365 & 0.36 \\
\hline & 1232 & $5.4268 \mathrm{E}-01$ & 0.827 & $1.0555 \mathrm{E}-01$ & 1.696 & 0.19 \\
\hline & 9000 & $2.8724 \mathrm{E}-01$ & 0.918 & $2.7479 \mathrm{E}-02$ & 1.941 & 0.10 \\
\hline & 68696 & $1.4675 \mathrm{E}-01$ & 0.969 & $6.9188 \mathrm{E}-03$ & 1.990 & 0.05 \\
\hline & 536760 & 7.3862E-02 & 0.990 & $1.7301 \mathrm{E}-03$ & 2.000 & 0.02 \\
\hline \multirow{6}{*}{$-10^{-2}$} & 26 & $1.5557 \mathrm{E}+00$ & & $3.5720 \mathrm{E}-01$ & & 0.23 \\
\hline & 180 & $1.0330 \mathrm{E}+00$ & 0.591 & $3.3360 \mathrm{E}-01$ & 0.099 & 0.32 \\
\hline & 1232 & $5.4634 \mathrm{E}-01$ & 0.919 & $1.0546 \mathrm{E}-01$ & 1.661 & 0.19 \\
\hline & 9000 & $2.8778 \mathrm{E}-01$ & 0.925 & $2.7478 \mathrm{E}-02$ & 1.940 & 0.10 \\
\hline & 68696 & $1.4683 \mathrm{E}-01$ & 0.971 & $6.9186 \mathrm{E}-03$ & 1.990 & 0.05 \\
\hline & 536760 & 7.3873E-02 & 0.991 & $1.7301 \mathrm{E}-03$ & 2.000 & 0.02 \\
\hline \multirow{6}{*}{$-10^{-4}$} & 26 & $1.5559 \mathrm{E}+00$ & & $3.5965 \mathrm{E}-01$ & & 0.23 \\
\hline & 180 & $3.4905 \mathrm{E}+01$ & -4.488 & $3.3361 \mathrm{E}-01$ & 0.108 & 0.01 \\
\hline & 1232 & $3.2421 \mathrm{E}+00$ & 3.428 & $1.0547 \mathrm{E}-01$ & 1.661 & 0.03 \\
\hline & 9000 & $3.7273 \mathrm{E}-01$ & 3.121 & $2.7479 \mathrm{E}-02$ & 1.940 & 0.07 \\
\hline & 68696 & $1.4775 \mathrm{E}-01$ & 1.335 & $6.9187 \mathrm{E}-03$ & 1.990 & 0.05 \\
\hline & 536760 & 7.3881E-02 & 1.000 & $1.7301 \mathrm{E}-03$ & 2.000 & 0.02 \\
\hline \multirow{6}{*}{$10^{-}$} & 26 & $1.5560 \mathrm{E}+00$ & & $3.5970 \mathrm{E}-01$ & & 0.23 \\
\hline & 180 & $3.4904 \mathrm{E}+01$ & -4.488 & $3.3361 \mathrm{E}-01$ & 0.109 & 0.01 \\
\hline & 1232 & $3.2420 \mathrm{E}+00$ & 3.428 & $1.0547 \mathrm{E}-01$ & 1.661 & 0.03 \\
\hline & 9000 & $3.7272 \mathrm{E}-01$ & 3.121 & $2.7479 \mathrm{E}-02$ & 1.940 & 0.07 \\
\hline & 68696 & $1.4775 \mathrm{E}-01$ & 1.335 & $6.9187 \mathrm{E}-03$ & 1.990 & 0.05 \\
\hline & 536760 & 7.3881E-02 & 1.000 & $1.7301 \mathrm{E}-03$ & 2.000 & 0.02 \\
\hline \multirow{6}{*}{$10^{-2}$} & 26 & $1.5562 \mathrm{E}+00$ & & $3.6218 \mathrm{E}-01$ & & 0.23 \\
\hline & 180 & $1.0329 \mathrm{E}+00$ & 0.591 & $3.3362 \mathrm{E}-01$ & 0.118 & 0.32 \\
\hline & 1232 & $5.4639 \mathrm{E}-01$ & 0.919 & $1.0549 \mathrm{E}-01$ & 1.661 & 0.19 \\
\hline & 9000 & $2.8779 \mathrm{E}-01$ & 0.925 & $2.7480 \mathrm{E}-02$ & 1.941 & 0.10 \\
\hline & 68696 & $1.4683 \mathrm{E}-01$ & 0.971 & $6.9188 \mathrm{E}-03$ & 1.990 & 0.05 \\
\hline & 536760 & 7.3873E-02 & 0.991 & $1.7301 \mathrm{E}-03$ & 2.000 & 0.02 \\
\hline \multirow{6}{*}{1} & 26 & $1.5958 \mathrm{E}+00$ & & $8.1746 \mathrm{E}-01$ & & 0.51 \\
\hline & 180 & $9.8448 \mathrm{E}-01$ & 0.697 & $3.5485 \mathrm{E}-01$ & 1.204 & 0.36 \\
\hline & 1232 & $5.4869 \mathrm{E}-01$ & 0.843 & $1.0841 \mathrm{E}-01$ & 1.711 & 0.20 \\
\hline & 9000 & $2.8838 \mathrm{E}-01$ & 0.928 & $2.7728 \mathrm{E}-02$ & 1.967 & 0.10 \\
\hline & 68696 & $1.4692 \mathrm{E}-01$ & 0.973 & $6.9365 \mathrm{E}-03$ & 1.999 & 0.05 \\
\hline & 536760 & 7.3885E-02 & 0.992 & $1.7313 \mathrm{E}-03$ & 2.002 & 0.02 \\
\hline
\end{tabular}

Table 6: Numerical errors and effectivity indices of the error estimator in $L^{2}$ norm (Uniform refinement). 


\section{A.2.2 Example 2}

We present, in Table 7, numerical errors and effectivity indices of the error estimator in $L^{2}$ norm for example 4.6.2. We can see unstable behavior of the convergence rates of $\|\mathbf{u}-\hat{\mathbf{u}}\|_{L^{2}(\Omega)}$ and $\|\varepsilon\|_{L^{2}(\Omega)}$ for all values of $\beta$.

As showen from these numerical data, the effectivities for the $L^{2}$ norm under first

kind elements are terrible, and are only shown to provide a contrast with the good effectivities for the second kind elements in the next section. 


\begin{tabular}{|c|c|c|c|c|c|c|}
\hline$\beta$ & $\operatorname{Dof}(V)$ & $\|\mathbf{u}-\hat{\mathbf{u}}\|_{L^{2}(\Omega)}$ & order & $\|\varepsilon\|_{L^{2}(\Omega)}$ & order & $\mathrm{EFF}$ \\
\hline \multirow{8}{*}{-1} & 26 & $2.9390 \mathrm{E}+00$ & & $1.1376 \mathrm{E}+00$ & & 0.39 \\
\hline & 180 & $2.7008 \mathrm{E}+00$ & -0.044 & $8.1395 \mathrm{E}-01$ & -0.173 & 0.30 \\
\hline & 1216 & $2.5136 \mathrm{E}+00$ & -0.038 & $3.5147 \mathrm{E}-01$ & -0.440 & 0.14 \\
\hline & 2852 & $8.5593 \mathrm{E}-01$ & -1.264 & $1.4117 \mathrm{E}-01$ & -1.070 & 0.16 \\
\hline & 4552 & $8.1450 \mathrm{E}-01$ & -0.106 & $1.0523 \mathrm{E}-01$ & -0.628 & 0.13 \\
\hline & 17076 & $5.2557 \mathrm{E}-01$ & -0.331 & $5.5874 \mathrm{E}-02$ & -0.479 & 0.11 \\
\hline & 45298 & $3.1545 \mathrm{E}-01$ & -0.523 & $2.5559 \mathrm{E}-02$ & -0.802 & 0.08 \\
\hline & 94716 & $2.7666 \mathrm{E}-01$ & -0.178 & $1.5933 \mathrm{E}-02$ & -0.641 & 0.06 \\
\hline \multirow{8}{*}{$-10^{-2}$} & 26 & $2.9481 \mathrm{E}+00$ & & $1.7718 \mathrm{E}+00$ & & 0.60 \\
\hline & 180 & $1.0493 \mathrm{E}+02$ & 1.846 & $1.0709 \mathrm{E}+00$ & -0.260 & 0.01 \\
\hline & 1216 & $2.0987 \mathrm{E}+02$ & 0.363 & $3.8198 \mathrm{E}-01$ & -0.540 & 0.00 \\
\hline & 2852 & $2.4466 \mathrm{E}+01$ & -2.521 & $1.4407 \mathrm{E}-01$ & -1.144 & 0.01 \\
\hline & 4552 & $4.3890 \mathrm{E}+01$ & 1.250 & $1.0682 \mathrm{E}-01$ & -0.640 & 0.00 \\
\hline & 15708 & $3.2474 \mathrm{E}+00$ & -2.102 & $6.1131 \mathrm{E}-02$ & -0.451 & 0.02 \\
\hline & 32368 & $7.0579 \mathrm{E}-01$ & -2.111 & $2.9981 \mathrm{E}-02$ & -0.985 & 0.04 \\
\hline & 103786 & $3.7042 \mathrm{E}-01$ & -0.553 & $1.5185 \mathrm{E}-02$ & -0.584 & 0.04 \\
\hline \multirow{8}{*}{$10^{-2}$} & 26 & $2.9483 \mathrm{E}+00$ & & $1.7885 \mathrm{E}+00$ & & 0.61 \\
\hline & 180 & $1.0493 \mathrm{E}+02$ & 1.846 & $1.0771 \mathrm{E}+00$ & -0.262 & 0.01 \\
\hline & 1216 & $2.0988 \mathrm{E}+02$ & 0.363 & $3.8264 \mathrm{E}-01$ & -0.542 & 0.00 \\
\hline & 2852 & $2.4468 \mathrm{E}+01$ & -2.521 & $1.4414 \mathrm{E}-01$ & -1.145 & 0.01 \\
\hline & 4552 & $4.3890 \mathrm{E}+01$ & 1.250 & $1.0686 \mathrm{E}-01$ & -0.640 & 0.00 \\
\hline & 15708 & $3.2474 \mathrm{E}+00$ & -2.102 & $6.1162 \mathrm{E}-02$ & -0.450 & 0.02 \\
\hline & 32368 & $7.0578 \mathrm{E}-01$ & -2.111 & $2.9984 \mathrm{E}-02$ & -0.986 & 0.04 \\
\hline & 103786 & $3.7042 \mathrm{E}-01$ & -0.553 & $1.5186 \mathrm{E}-02$ & -0.584 & 0.04 \\
\hline \multirow{8}{*}{1} & 26 & $2.9644 \mathrm{E}+00$ & & $2.9746 \mathrm{E}+00$ & & 1.00 \\
\hline & 180 & $2.7281 \mathrm{E}+00$ & -0.043 & $1.4569 \mathrm{E}+00$ & -0.369 & 0.53 \\
\hline & 1168 & $2.5358 \mathrm{E}+00$ & -0.039 & 4.1993E-01 & -0.665 & 0.17 \\
\hline & 2804 & $8.5821 \mathrm{E}-01$ & -1.237 & $1.5631 \mathrm{E}-01$ & -1.128 & 0.18 \\
\hline & 4504 & $8.1576 \mathrm{E}-01$ & -0.107 & $1.2067 \mathrm{E}-01$ & -0.546 & 0.15 \\
\hline & 15048 & $5.4365 \mathrm{E}-01$ & -0.336 & $6.4376 \mathrm{E}-02$ & -0.521 & 0.12 \\
\hline & 31852 & $3.7034 \mathrm{E}-01$ & -0.512 & $3.1309 \mathrm{E}-02$ & -0.961 & 0.08 \\
\hline & 95494 & $2.6700 \mathrm{E}-01$ & -0.298 & $1.5915 \mathrm{E}-02$ & -0.616 & 0.06 \\
\hline
\end{tabular}

Table 7: Numerical errors and effectivity indices of the error estimator in $L^{2}$ norm (Adaptive refinement.) 


\section{A.3 Numerical Results by using Nédélec space of the second-kind}

In the second section, we will resolve the problems in examples 4.6 .1 and 4.6 .2 by using first-order Nédélec space of the second-kind and then we report errors and effectivities in the global $H$-curl norm, energy norm, and $L^{2}$ norm. Also, we calculate condition numbers for the matrices used to compute $\hat{\mathbf{u}}$ and $\varepsilon$. First, we start by presenting the local $p$-order Nédélec space of the second-kind and its finite element.

\section{A.3.1 Nédélec $H(\operatorname{curl} ; \Omega)$ element of the second-kind}

For any given conforming tetrahedral triangulation $\mathcal{T}$ of $\Omega$, we define below the local $p$-order Nédélec space of the second-kind. But first we need to define the spaces $D_{p}(T)$ and $D_{p}(F)$ that used for the definition of Nédélec space of the second-kind.

$$
\begin{array}{ll}
D_{p}(T)=\left[\mathbb{P}_{p-1}\right]^{3} \oplus \tilde{\mathbb{P}}_{p-1} \boldsymbol{x}_{\boldsymbol{T}} \quad, \quad \operatorname{dim} D_{p}=1 / 2 p(p+1)(p+3), \\
D_{p}(F)=\left[\mathbb{P}_{p-1}\right]^{2} \oplus \tilde{\mathbb{P}}_{p-1} \boldsymbol{x}_{\boldsymbol{F}} \quad, \quad \operatorname{dim} D_{p}=p(p+2),
\end{array}
$$

where $\boldsymbol{x}_{\boldsymbol{T}} \in \mathbb{R}^{3}$ and $\boldsymbol{x}_{\boldsymbol{F}} \in \mathbb{R}^{2}$.

Definition .1. The curl-conforming finite element of Nédélec of the second-kind is defined by:

- $T$ is a tetrahedron,

- $V_{p}(T)=\left[\mathbb{P}_{p}\right]^{3}$,

- The degrees of freedom are of three types associated with edges e of T, faces $F$ of $T$ and $T$ itself. We denote by $\mathbf{t}$ a unit vector in the direction of the edge $e$. There are three different degrees of freedom: 
1. the first set is associated with edges of the element:

$$
M_{e}(\mathbf{v})=\left\{\int_{e}(\mathbf{v} \cdot \mathbf{t}) q d s \text { for all } q \in \mathbb{P}_{p}(e) \text { and } e \in \mathcal{E}_{T}\right\}
$$

2. the second set is associated with faces of the element:

$$
M_{F}(\mathbf{v})=\left\{\int_{F_{k}} \mathbf{v} \cdot \mathbf{q} d A \text { for all } \mathbf{q} \in D_{p-1}(F) \text { and } F \in \mathcal{F}\right\}
$$

3. the last set is associated with the volume:

$$
M_{T}(\mathbf{v})=\left\{\int_{T} \mathbf{v} \cdot \mathbf{q} d V \text { for all } \mathbf{q} \in D_{p-2}(T)\right\} .
$$

Then $\Sigma_{T}=M_{e}(\mathbf{v}) \cup M_{F}(\mathbf{v}) \cup M_{T}(\mathbf{v})$.

In the computation, we used the second-kind Nédélec space of lowest order, $V_{1}$, to compute $\hat{\mathbf{u}}$. Then we choose the decomposition of second and third order secondkind Nédélec spaces $\left(W(T)=V_{2}^{F}(T) \oplus V_{3}^{T}(T)\right)$ to compute the error function $\varepsilon$. This means that the auxiliary subspace $W$ has the same form in first-kind Nédélec space but its components have different definitions and dimensions based on the definitions above. So the global finite error space $W$ and its dimension are given by

$$
\begin{aligned}
W & =\left\{\mathbf{v} \in \mathcal{H}:\left.\mathbf{v}\right|_{T} \in V_{2}^{F}(T) \oplus V_{3}^{T}(T) \text { for all } T \in \mathcal{T}\right\}, \\
\operatorname{dim} W & =3 \# \mathcal{F}+4 \# \mathcal{T} .
\end{aligned}
$$

\section{A.3.2 Example 1}

Here we presents numerical errors, convergence rates, and effectivities for the problem in example 4.6.1. In Table 8, we show errors in $L^{2}$ norm. The rate of convergence is $O\left(h^{2}\right)$ when $\beta= \pm 1$ and $O\left(h^{5 / 2}\right)$ when $\beta=\left\{ \pm 10^{-2}, \pm 10^{-4}\right\}$. The effectivity indices approach a stable constant rapidly as refinement proceeds for most values of $\beta$. Results in $H$-curl norm are showen in Table 9. Here we have convergence rate of 
$O(h)$ for $\beta \in\left\{ \pm 1, \pm 10^{-2}\right\}$ and $O\left(h^{5 / 2}\right)$ when $\beta=\left\{ \pm 10^{-4}\right\}$, and the effectivity indices fastly approach a stable value. Errors in energy norms are presented in Table 10 . From the data in this table, we observe that, the exact error and approximate error are achieve optimal convergence rates, $O(h)$, and the effectivity indices approach a stable constant, 0.96, rapidly as refinement proceeds for all values of $\beta$.

In Table 11, we present the condition numbers of the diagonally-rescaled stiffness matrices for $W$ and $V$ with respect to the number of degrees of freedom on different mesh refinement levels. We were able to get condition numbers for just cases when values of $\beta$ are negative. From this, we notice that the computed condition numbers for stiffness matrix for $W$ are stable and bounded in short range [24: 150]. However, the condition numbers for stiffness matrix for $V$ grows as the mesh is refined. These results show that the matrix associated with the computation of $\varepsilon$ is much better conditioned, which is certainly not the case for the matrix associated with computing $\hat{\mathbf{u}}$ on $V$. 


\begin{tabular}{c||l|ll|cc|c}
\hline$\beta$ & $D o f(V)$ & $\|\mathbf{u}-\hat{\mathbf{u}}\|_{L^{2}(\Omega)}$ & order & $\|\varepsilon\|_{L^{2}(\Omega)}$ & order & EFF \\
\hline \multirow{5}{*}{-1} & 52 & $5.0773 \mathrm{E}-01$ & & $3.6569 \mathrm{E}-01$ & & 0.72 \\
& 334 & $3.8117 \mathrm{E}-01$ & 0.414 & $3.3079 \mathrm{E}-01$ & 0.145 & 0.87 \\
& 2284 & $1.2467 \mathrm{E}-01$ & 1.612 & $1.0719 \mathrm{E}-01$ & 1.626 & 0.86 \\
& 16768 & $3.3439 \mathrm{E}-02$ & 1.898 & $2.8855 \mathrm{E}-02$ & 1.893 & 0.86 \\
& 128392 & $8.5378 \mathrm{E}-03$ & 1.970 & $7.4056 \mathrm{E}-03$ & 1.962 & 0.87 \\
\hline \multirow{5}{*}{$-10^{-2}$} & 52 & $1.7909 \mathrm{E}+01$ & & $1.7903 \mathrm{E}+01$ & & 1.00 \\
& 334 & $6.3839 \mathrm{E}+00$ & 1.488 & $6.2799 \mathrm{E}+00$ & 1.511 & 0.98 \\
& 2284 & $1.4966 \mathrm{E}+00$ & 2.093 & $1.4603 \mathrm{E}+00$ & 2.104 & 0.98 \\
& 16768 & $2.8427 \mathrm{E}-01$ & 2.396 & $2.7676 \mathrm{E}-01$ & 2.400 & 0.97 \\
& 128392 & $5.1267 \mathrm{E}-02$ & 2.471 & $4.9785 \mathrm{E}-02$ & 2.475 & 0.97 \\
\hline \multirow{5}{*}{$-10^{-4}$} & 52 & $1.7868 \mathrm{E}+03$ & & $1.7868 \mathrm{E}+03$ & & 1.00 \\
& 334 & $6.3704 \mathrm{E}+02$ & 1.488 & $6.2675 \mathrm{E}+02$ & 1.511 & 0.98 \\
& 2284 & $1.4900 \mathrm{E}+02$ & 2.096 & $1.4550 \mathrm{E}+02$ & 2.107 & 0.98 \\
& 16768 & $2.8189 \mathrm{E}+01$ & 2.402 & $2.7509 \mathrm{E}+01$ & 2.403 & 0.98 \\
& 128392 & $5.0431 \mathrm{E}+00$ & 2.483 & $4.9211 \mathrm{E}+00$ & 2.483 & 0.98 \\
\hline \multirow{5}{*}{$10^{-4}$} & 52 & $1.7867 \mathrm{E}+03$ & & $1.7867 \mathrm{E}+03$ & & 1.00 \\
& 334 & $6.3704 \mathrm{E}+02$ & 1.488 & $6.2674 \mathrm{E}+02$ & 1.511 & 0.98 \\
& 2284 & $1.4900 \mathrm{E}+02$ & 2.096 & $1.4550 \mathrm{E}+02$ & 2.107 & 0.98 \\
& 16768 & $2.8189 \mathrm{E}+01$ & 2.402 & $2.7509 \mathrm{E}+01$ & 2.403 & 0.98 \\
& 128392 & $5.0430 \mathrm{E}+00$ & 2.483 & $4.9211 \mathrm{E}+00$ & 2.483 & 0.98 \\
\hline \multirow{5}{*}{$10^{-2}$} & 52 & $1.7841 \mathrm{E}+01$ & & $1.7836 \mathrm{E}+01$ & & 1.00 \\
& 334 & $6.3818 \mathrm{E}+00$ & 1.483 & $6.2725 \mathrm{E}+00$ & 1.508 & 0.98 \\
& 2284 & $1.4952 \mathrm{E}+00$ & 2.094 & $1.4576 \mathrm{E}+00$ & 2.105 & 0.97 \\
& 16768 & $2.8402 \mathrm{E}-01$ & 2.396 & $2.7642 \mathrm{E}-01$ & 2.399 & 0.97 \\
& 128392 & $5.1232 \mathrm{E}-02$ & 2.471 & $4.9742 \mathrm{E}-02$ & 2.474 & 0.97 \\
\hline \multirow{5}{*}{} & 52 & $5.8764 \mathrm{E}-01$ & & $3.4525 \mathrm{E}-01$ & & 0.59 \\
& 334 & $4.3873 \mathrm{E}-01$ & 0.422 & $3.4190 \mathrm{E}-01$ & 0.014 & 0.78 \\
& 2284 & $1.4673 \mathrm{E}-01$ & 1.580 & $1.0852 \mathrm{E}-01$ & 1.656 & 0.74 \\
& 16768 & $3.9584 \mathrm{E}-02$ & 1.890 & $2.8974 \mathrm{E}-02$ & 1.905 & 0.73 \\
& 128392 & $1.0093 \mathrm{E}-02$ & 1.972 & $7.4143 \mathrm{E}-03$ & 1.966 & 0.73 \\
\hline \multirow{5}{*}{} & & & & & &
\end{tabular}

Table 8: Numerical errors and effectivity indices of the error estimator in $L^{2}$ norm (Uniform refinement). 


\begin{tabular}{|c|c|c|c|c|c|c|}
\hline$\beta$ & $\operatorname{Dof}(V)$ & $\|\mathbf{u}-\hat{\mathbf{u}}\|_{H(\operatorname{curl} ; \Omega)}$ & order & $\|\varepsilon\|_{H(\operatorname{curl} ; \Omega)}$ & order & EFF \\
\hline \multirow{5}{*}{-1} & 52 & $2.3322 \mathrm{E}+00$ & & $1.5391 \mathrm{E}+00$ & & 0.66 \\
\hline & 334 & $1.8977 \mathrm{E}+00$ & 0.297 & $1.7446 \mathrm{E}+00$ & -0.181 & 0.92 \\
\hline & 2284 & $1.0680 \mathrm{E}+00$ & 0.829 & $1.0156 \mathrm{E}+00$ & 0.781 & 0.95 \\
\hline & 16768 & $5.4585 \mathrm{E}-01$ & 0.968 & $5.2422 \mathrm{E}-01$ & 0.954 & 0.96 \\
\hline & 128392 & $2.7331 \mathrm{E}-01$ & 0.998 & $2.6286 \mathrm{E}-01$ & 0.996 & 0.96 \\
\hline \multirow{5}{*}{$-10^{-2}$} & 52 & $1.8051 \mathrm{E}+01$ & & $1.7963 \mathrm{E}+01$ & & 1.00 \\
\hline & 334 & $6.6484 \mathrm{E}+00$ & 1.441 & $6.5086 \mathrm{E}+00$ & 1.465 & 0.98 \\
\hline & 2284 & $1.8341 \mathrm{E}+00$ & 1.858 & $1.7749 \mathrm{E}+00$ & 1.875 & 0.97 \\
\hline & 16768 & $6.1446 \mathrm{E}-01$ & 1.578 & $5.9196 \mathrm{E}-01$ & 1.584 & 0.96 \\
\hline & 128392 & 2.7793E-01 & 1.145 & $2.6741 \mathrm{E}-01$ & 1.146 & 0.96 \\
\hline \multirow{5}{*}{$-10^{-4}$} & 52 & $1.7868 \mathrm{E}+03$ & & $1.7868 \mathrm{E}+03$ & & 1.00 \\
\hline & 334 & $6.3704 \mathrm{E}+02$ & 1.488 & $6.2675 \mathrm{E}+02$ & 1.511 & 0.98 \\
\hline & 2284 & $1.4901 \mathrm{E}+02$ & 2.096 & $1.4551 \mathrm{E}+02$ & 2.107 & 0.98 \\
\hline & 16768 & $2.8195 \mathrm{E}+01$ & 2.402 & $2.7514 \mathrm{E}+01$ & 2.403 & 0.98 \\
\hline & 128392 & $5.0504 \mathrm{E}+00$ & 2.481 & $4.9281 \mathrm{E}+00$ & 2.481 & 0.98 \\
\hline \multirow{5}{*}{$10^{-4}$} & 52 & $1.7867 \mathrm{E}+03$ & & $1.7867 \mathrm{E}+03$ & & 1.00 \\
\hline & 334 & $6.3704 \mathrm{E}+02$ & 1.488 & $6.2674 \mathrm{E}+02$ & 1.511 & 0.98 \\
\hline & 2284 & $1.4901 \mathrm{E}+02$ & 2.096 & $1.4551 \mathrm{E}+02$ & 2.107 & 0.98 \\
\hline & 16768 & $2.8194 \mathrm{E}+01$ & 2.402 & $2.7514 \mathrm{E}+01$ & 2.403 & 0.98 \\
\hline & 128392 & $5.0504 \mathrm{E}+00$ & 2.481 & $4.9281 \mathrm{E}+00$ & 2.481 & 0.98 \\
\hline \multirow{5}{*}{$10^{-2}$} & 52 & $1.7983 \mathrm{E}+01$ & & $1.7897 \mathrm{E}+01$ & & 1.00 \\
\hline & 334 & $6.6464 \mathrm{E}+00$ & 1.436 & $6.5015 \mathrm{E}+00$ & 1.461 & 0.98 \\
\hline & 2284 & $1.8329 \mathrm{E}+00$ & 1.858 & $1.7727 \mathrm{E}+00$ & 1.875 & 0.97 \\
\hline & 16768 & $6.1435 \mathrm{E}-01$ & 1.577 & $5.9180 \mathrm{E}-01$ & 1.583 & 0.96 \\
\hline & 128392 & $2.7793 \mathrm{E}-01$ & 1.144 & $2.6740 \mathrm{E}-01$ & 1.146 & 0.96 \\
\hline \multirow{5}{*}{1} & 52 & $2.3358 \mathrm{E}+00$ & & $1.4626 \mathrm{E}+00$ & & 0.63 \\
\hline & 334 & $1.9114 \mathrm{E}+00$ & 0.289 & $1.7340 \mathrm{E}+00$ & -0.246 & 0.91 \\
\hline & 2284 & $1.0713 \mathrm{E}+00$ & 0.835 & $1.0114 \mathrm{E}+00$ & 0.778 & 0.94 \\
\hline & 16768 & $5.4634 \mathrm{E}-01$ & 0.971 & $5.2356 \mathrm{E}-01$ & 0.950 & 0.96 \\
\hline & 128392 & $2.7337 \mathrm{E}-01$ & 0.999 & $2.6278 \mathrm{E}-01$ & 0.995 & 0.96 \\
\hline
\end{tabular}

Table 9: Numerical errors, convergence order, and effectivity indices of the error estimator in $H$-curl norm (Uniform refinement). 


\begin{tabular}{c||l|cc|cc|c}
\hline$\beta$ & $D o f(V)$ & $\|\mathbf{u}-\hat{\mathbf{u}}\|$ & order & $\|\varepsilon\|$ & order & EFF \\
\hline \multirow{5}{*}{-1} & 52 & $2.3322 \mathrm{E}+00$ & & $1.5391 \mathrm{E}+00$ & & 0.66 \\
& 334 & $1.8977 \mathrm{E}+00$ & 0.297 & $1.7446 \mathrm{E}+00$ & -0.181 & 0.92 \\
& 2284 & $1.0680 \mathrm{E}+00$ & 0.829 & $1.0156 \mathrm{E}+00$ & 0.781 & 0.95 \\
& 16768 & $5.4585 \mathrm{E}-01$ & 0.968 & $5.2422 \mathrm{E}-01$ & 0.954 & 0.96 \\
& 128392 & $2.7331 \mathrm{E}-01$ & 0.998 & $2.6286 \mathrm{E}-01$ & 0.996 & 0.96 \\
\hline \multirow{5}{*}{$-10^{-2}$} & 52 & $2.8848 \mathrm{E}+00$ & & $2.3142 \mathrm{E}+00$ & & 0.80 \\
& 334 & $1.9632 \mathrm{E}+00$ & 0.555 & $1.8220 \mathrm{E}+00$ & 0.345 & 0.93 \\
& 2284 & $1.0707 \mathrm{E}+00$ & 0.875 & $1.0194 \mathrm{E}+00$ & 0.838 & 0.95 \\
& 16768 & $5.4550 \mathrm{E}-01$ & 0.973 & $5.2401 \mathrm{E}-01$ & 0.960 & 0.96 \\
& 128392 & $2.7321 \mathrm{E}-01$ & 0.998 & $2.6278 \mathrm{E}-01$ & 0.996 & 0.96 \\
\hline \multirow{5}{*}{$-10^{-4}$} & 52 & $1.8011 \mathrm{E}+01$ & & $1.7928 \mathrm{E}+01$ & & 1.00 \\
& 334 & $6.6354 \mathrm{E}+00$ & 1.441 & $6.4967 \mathrm{E}+00$ & 1.464 & 0.98 \\
& 2284 & $1.8287 \mathrm{E}+00$ & 1.859 & $1.7706 \mathrm{E}+00$ & 1.875 & 0.97 \\
& 16768 & $6.1337 \mathrm{E}-01$ & 1.576 & $5.9118 \mathrm{E}-01$ & 1.583 & 0.96 \\
& 128392 & $2.7778 \mathrm{E}-01$ & 1.143 & $2.6731 \mathrm{E}-01$ & 1.145 & 0.96 \\
\hline \multirow{5}{*}{$10^{-4}$} & 52 & $1.8010 \mathrm{E}+01$ & & $1.7927 \mathrm{E}+01$ & & 1.00 \\
& 334 & $6.6354 \mathrm{E}+00$ & 1.441 & $6.4966 \mathrm{E}+00$ & 1.464 & 0.98 \\
& 2284 & $1.8287 \mathrm{E}+00$ & 1.859 & $1.7706 \mathrm{E}+00$ & 1.875 & 0.97 \\
& 16768 & $6.1337 \mathrm{E}-01$ & 1.576 & $5.9118 \mathrm{E}-01$ & 1.583 & 0.96 \\
& 128392 & $2.7778 \mathrm{E}-01$ & 1.143 & $2.6731 \mathrm{E}-01$ & 1.145 & 0.96 \\
\hline \multirow{5}{*}{$10^{-2}$} & 52 & $1.7983 \mathrm{E}+01$ & & $1.7897 \mathrm{E}+01$ & & 1.00 \\
& 334 & $6.6464 \mathrm{E}+00$ & 1.436 & $6.5015 \mathrm{E}+00$ & 1.461 & 0.98 \\
& 2284 & $1.8329 \mathrm{E}+00$ & 1.858 & $1.7727 \mathrm{E}+00$ & 1.875 & 0.97 \\
& 16768 & $6.1435 \mathrm{E}-01$ & 1.577 & $5.9180 \mathrm{E}-01$ & 1.583 & 0.96 \\
& 128392 & $2.7793 \mathrm{E}-01$ & 1.144 & $2.6740 \mathrm{E}-01$ & 1.146 & 0.96 \\
\hline \multirow{5}{*}{1} & 52 & $2.3358 \mathrm{E}+00$ & & $1.4626 \mathrm{E}+00$ & & 0.63 \\
& 334 & $1.9114 \mathrm{E}+00$ & 0.289 & $1.7340 \mathrm{E}+00$ & -0.246 & 0.91 \\
& 2284 & $1.0713 \mathrm{E}+00$ & 0.835 & $1.0114 \mathrm{E}+00$ & 0.778 & 0.94 \\
& 16768 & $5.4634 \mathrm{E}-01$ & 0.971 & $5.2356 \mathrm{E}-01$ & 0.950 & 0.96 \\
& 128392 & $2.7337 \mathrm{E}-01$ & 0.999 & $2.6278 \mathrm{E}-01$ & 0.995 & 0.96 \\
\hline \multirow{5}{*}{} & & & & & & \\
& & & & & \\
& & & & & &
\end{tabular}

Table 10: Numerical errors, convergence order, and effectivity indices of the error estimator in energy norm (Uniform refinement). 


\begin{tabular}{c||l|l|l}
\hline$\beta$ & $\operatorname{Dof}(V)$ & Cnd. No $(V)$ & Cnd. No $(W)$ \\
\hline \multirow{5}{*}{-1} & 52 & 27.571 & 30.882 \\
& 334 & 201.776 & 57.482 \\
& 2284 & 587.814 & 94.600 \\
& 16768 & 1306.276 & 132.515 \\
& 128392 & 4575.384 & 150.561 \\
\hline \multirow{5}{*}{$-10^{-2}$} & 52 & 2228.669 & 24.427 \\
& 334 & 15345.589 & 54.058 \\
& 2284 & 31840.001 & 93.343 \\
& 16768 & 91092.717 & 131.897 \\
& 128392 & 97016.678 & 149.811 \\
\hline \multirow{5}{*}{$-10^{-4}$} & 52 & 222345.435 & 24.284 \\
& 334 & 930557.758 & 54.017 \\
& 2284 & 2520070.668 & 93.329 \\
& 16768 & 232300.082 & 131.837 \\
& 128392 & 54698.544 & 149.653 \\
\hline
\end{tabular}

Table 11: Condition numbers of the stiffness matrices for $V$ and $W$ (Uniform refinement).

\section{A.3.3 Example 2}

In this section, we resolve the problem in example 4.6.2 by using first-order Nédélec space of the second-kind. We show the numerical errors in $L^{2}$ norm, $H$-curl norm, and energy norm in Tables 12 , 13, and 14, respectively. From these tables, we observe that, in all norm types and most cases of $\beta$, the effectivity indices fastly approach a stable value despite the erratic behavior of the convergence rate of the errors.

The condition numbers of the diagonally-rescaled stiffness matrices for $W$ and $V$ are showen in Table 15 . For negative values of $\beta$, the computed condition numbers for stiffness matrix for $W$ remained stable and bounded in the range [24:173]. However, the condition numbers for stiffness matrix for $V$ are growing as the mesh is adaptively refined, and their ranges vary based on the difference of $\beta$ values. For positive values of $\beta$, no results were obtained for condition numbers of both stiffness matrices for $W$ 
and $V$.

\begin{tabular}{c||l|cc|cc|c}
\hline$\beta$ & $D o f(V)$ & $\|\mathbf{u}-\hat{\mathbf{u}}\|_{L^{2}(\Omega)}$ & order & $\|\varepsilon\|_{L^{2}(\Omega)}$ & order & EFF \\
\hline \multirow{5}{*}{-1} & 52 & $4.5522 \mathrm{E}+00$ & & $4.1301 \mathrm{E}+00$ & & 0.91 \\
& 334 & $6.2400 \mathrm{E}+00$ & 0.170 & $4.6333 \mathrm{E}+00$ & 0.062 & 0.74 \\
& 2164 & $8.5726 \mathrm{E}+00$ & 0.170 & $8.4281 \mathrm{E}+00$ & 0.320 & 0.98 \\
& 5218 & $2.3684 \mathrm{E}+00$ & -1.461 & $2.3842 \mathrm{E}+00$ & -1.435 & 1.01 \\
& 8272 & $1.6801 \mathrm{E}+00$ & -0.745 & $1.6547 \mathrm{E}+00$ & -0.793 & 0.98 \\
& 11326 & $3.1887 \mathrm{E}-01$ & -5.289 & $2.9509 \mathrm{E}-01$ & -5.487 & 0.93 \\
& 21856 & $1.8479 \mathrm{E}-01$ & -0.830 & $1.6952 \mathrm{E}-01$ & -0.843 & 0.92 \\
& 70138 & $1.0442 \mathrm{E}-01$ & -0.490 & $9.8537 \mathrm{E}-02$ & -0.465 & 0.94 \\
& 151174 & $5.3707 \mathrm{E}-02$ & -0.866 & $5.0636 \mathrm{E}-02$ & -0.867 & 0.94 \\
\hline \multirow{5}{*}{$10^{-2}$} & 52 & $4.3348 \mathrm{E}+02$ & & $4.2840 \mathrm{E}+02$ & & 0.99 \\
& 334 & $6.0895 \mathrm{E}+02$ & 0.183 & $4.6839 \mathrm{E}+02$ & 0.048 & 0.77 \\
& 2164 & $8.5548 \mathrm{E}+02$ & 0.182 & $8.4367 \mathrm{E}+02$ & 0.315 & 0.99 \\
& 5218 & $2.3663 \mathrm{E}+02$ & -1.460 & $2.3866 \mathrm{E}+02$ & -1.435 & 1.01 \\
& 7936 & $1.6701 \mathrm{E}+02$ & -0.831 & $1.6477 \mathrm{E}+02$ & -0.884 & 0.99 \\
& 11326 & $2.7217 \mathrm{E}+01$ & -5.101 & $2.6762 \mathrm{E}+01$ & -5.110 & 0.98 \\
& 14380 & $1.4132 \mathrm{E}+01$ & -2.745 & $1.3716 \mathrm{E}+01$ & -2.800 & 0.97 \\
& 52780 & $6.5905 \mathrm{E}+00$ & -0.587 & $6.4269 \mathrm{E}+00$ & -0.583 & 0.98 \\
& 74656 & $4.7801 \mathrm{E}+00$ & -0.926 & $4.6857 \mathrm{E}+00$ & -0.911 & 0.98 \\
\hline \multirow{5}{*}{$10^{-2}$} & 52 & $4.3380 \mathrm{E}+02$ & & $4.2887 \mathrm{E}+02$ & & 0.99 \\
& 334 & $6.0895 \mathrm{E}+02$ & 0.182 & $4.6855 \mathrm{E}+02$ & 0.048 & 0.77 \\
& 2164 & $8.5549 \mathrm{E}+02$ & 0.182 & $8.4369 \mathrm{E}+02$ & 0.315 & 0.99 \\
& 5218 & $2.3664 \mathrm{E}+02$ & -1.460 & $2.3868 \mathrm{E}+02$ & -1.435 & 1.01 \\
& 7936 & $1.6700 \mathrm{E}+02$ & -0.831 & $1.6476 \mathrm{E}+02$ & -0.884 & 0.99 \\
& 11326 & $2.7216 \mathrm{E}+01$ & -5.101 & $2.6761 \mathrm{E}+01$ & -5.110 & 0.98 \\
& 14380 & $1.4132 \mathrm{E}+01$ & -2.745 & $1.3716 \mathrm{E}+01$ & -2.800 & 0.97 \\
& 52780 & $6.5897 \mathrm{E}+00$ & -0.587 & $6.4260 \mathrm{E}+00$ & -0.583 & 0.98 \\
& 74656 & $4.7800 \mathrm{E}+00$ & -0.926 & $4.6857 \mathrm{E}+00$ & -0.911 & 0.98 \\
\hline \multirow{5}{*}{} & 52 & $5.0018 \mathrm{E}+00$ & & $4.5822 \mathrm{E}+00$ & & 0.92 \\
& 334 & $6.3247 \mathrm{E}+00$ & 0.126 & $4.7948 \mathrm{E}+00$ & 0.024 & 0.76 \\
& 2164 & $8.5994 \mathrm{E}+00$ & 0.164 & $8.4517 \mathrm{E}+00$ & 0.303 & 0.98 \\
& 5218 & $2.3825 \mathrm{E}+00$ & -1.458 & $2.3974 \mathrm{E}+00$ & -1.432 & 1.01 \\
& 11326 & $1.6772 \mathrm{E}+00$ & -0.762 & $1.6497 \mathrm{E}+00$ & -0.811 & 0.98 \\
& 21856 & $1.2785 \mathrm{E}-01$ & -5.195 & $2.9470 \mathrm{E}-01$ & -5.481 & 0.90 \\
& 70810 & $1.036158 \mathrm{E}-01$ & -0.841 & $1.6944 \mathrm{E}-01$ & -0.842 & 0.90 \\
& & $5.5125 \mathrm{E}-02$ & -0.845 & $5.0824 \mathrm{E}-02$ & -0.848 & 0.92 \\
\hline
\end{tabular}

Table 12: Numerical errors and effectivity indices of the error estimator in $L^{2}$ norm (Adaptive refinement). 


\begin{tabular}{|c|c|c|c|c|c|c|}
\hline$\beta$ & $\operatorname{Dof}(V)$ & $\|\mathbf{u}-\hat{\mathbf{u}}\|_{H(\operatorname{curl} ; \Omega)}$ & order & $\|\varepsilon\|_{H(\operatorname{curl} ; \Omega)}$ & order & $\mathrm{EFF}$ \\
\hline \multirow{9}{*}{-1} & 52 & $8.4612 \mathrm{E}+00$ & & $5.7291 \mathrm{E}+00$ & & 0.68 \\
\hline & 334 & $8.6984 \mathrm{E}+00$ & 0.015 & $5.7495 \mathrm{E}+00$ & 0.002 & 0.66 \\
\hline & 2164 & $9.7104 \mathrm{E}+00$ & 0.059 & $8.9312 \mathrm{E}+00$ & 0.236 & 0.92 \\
\hline & 5218 & $3.5941 \mathrm{E}+00$ & -1.129 & $3.3856 \mathrm{E}+00$ & -1.102 & 0.94 \\
\hline & 8272 & $2.6783 \mathrm{E}+00$ & -0.638 & $2.5916 \mathrm{E}+00$ & -0.580 & 0.97 \\
\hline & 11326 & $1.9417 \mathrm{E}+00$ & -1.023 & $1.8669 \mathrm{E}+00$ & -1.044 & 0.96 \\
\hline & 21856 & $1.5911 \mathrm{E}+00$ & -0.303 & $1.5379 \mathrm{E}+00$ & -0.295 & 0.97 \\
\hline & 70138 & $1.0471 \mathrm{E}+00$ & -0.359 & $1.0163 \mathrm{E}+00$ & -0.355 & 0.97 \\
\hline & 151174 & $8.1930 \mathrm{E}-01$ & -0.319 & $7.9837 \mathrm{E}-01$ & -0.314 & 0.97 \\
\hline \multirow{9}{*}{$-10^{-2}$} & 52 & $4.3354 \mathrm{E}+02$ & & $4.2842 \mathrm{E}+02$ & & 0.99 \\
\hline & 334 & $6.0898 \mathrm{E}+02$ & 0.183 & $4.6840 \mathrm{E}+02$ & 0.048 & 0.77 \\
\hline & 2164 & $8.5549 \mathrm{E}+02$ & 0.182 & $8.4367 \mathrm{E}+02$ & 0.315 & 0.99 \\
\hline & 5218 & $2.3665 \mathrm{E}+02$ & -1.460 & $2.3868 \mathrm{E}+02$ & -1.435 & 1.01 \\
\hline & 7936 & $1.6702 \mathrm{E}+02$ & -0.831 & $1.6478 \mathrm{E}+02$ & -0.884 & 0.99 \\
\hline & 11326 & $2.7284 \mathrm{E}+01$ & -5.094 & $2.6825 \mathrm{E}+01$ & -5.104 & 0.98 \\
\hline & 14380 & $1.4256 \mathrm{E}+01$ & -2.719 & $1.3834 \mathrm{E}+01$ & -2.774 & 0.97 \\
\hline & 52780 & $6.7541 \mathrm{E}+00$ & -0.574 & $6.5835 \mathrm{E}+00$ & -0.571 & 0.97 \\
\hline & 74656 & $4.9329 \mathrm{E}+00$ & -0.906 & $4.8336 \mathrm{E}+00$ & -0.891 & 0.98 \\
\hline \multirow{9}{*}{$10^{-2}$} & 52 & $4.3387 \mathrm{E}+02$ & & $4.2888 \mathrm{E}+02$ & & 0.99 \\
\hline & 334 & $6.0899 \mathrm{E}+02$ & 0.182 & $4.6856 \mathrm{E}+02$ & 0.048 & 0.77 \\
\hline & 2164 & $8.5550 \mathrm{E}+02$ & 0.182 & $8.4369 \mathrm{E}+02$ & 0.315 & 0.99 \\
\hline & 5218 & $2.3666 \mathrm{E}+02$ & -1.460 & $2.3869 \mathrm{E}+02$ & -1.435 & 1.01 \\
\hline & 7936 & $1.6702 \mathrm{E}+02$ & -0.831 & $1.6477 \mathrm{E}+02$ & -0.884 & 0.99 \\
\hline & 11326 & $2.7283 \mathrm{E}+01$ & -5.094 & $2.6825 \mathrm{E}+01$ & -5.103 & 0.98 \\
\hline & 14380 & $1.4255 \mathrm{E}+01$ & -2.719 & $1.3833 \mathrm{E}+01$ & -2.774 & 0.97 \\
\hline & 52780 & $6.7534 \mathrm{E}+00$ & -0.575 & $6.5827 \mathrm{E}+00$ & -0.571 & 0.97 \\
\hline & 74656 & $4.9328 \mathrm{E}+00$ & -0.906 & $4.8335 \mathrm{E}+00$ & -0.891 & 0.98 \\
\hline \multirow{9}{*}{1} & 52 & $9.0601 \mathrm{E}+00$ & & $5.9403 \mathrm{E}+00$ & & 0.66 \\
\hline & 334 & $9.0556 \mathrm{E}+00$ & -0.000 & $5.7831 \mathrm{E}+00$ & -0.014 & 0.64 \\
\hline & 2164 & $9.7985 \mathrm{E}+00$ & 0.042 & $8.9340 \mathrm{E}+00$ & 0.233 & 0.91 \\
\hline & 5218 & $3.6038 \mathrm{E}+00$ & -1.136 & $3.3878 \mathrm{E}+00$ & -1.102 & 0.94 \\
\hline & 8272 & $2.6757 \mathrm{E}+00$ & -0.646 & $2.5827 \mathrm{E}+00$ & -0.589 & 0.97 \\
\hline & 11326 & $1.9435 \mathrm{E}+00$ & -1.018 & $1.8580 \mathrm{E}+00$ & -1.048 & 0.96 \\
\hline & 21856 & $1.5916 \mathrm{E}+00$ & -0.304 & $1.5350 \mathrm{E}+00$ & -0.290 & 0.96 \\
\hline & 70810 & $1.0419 \mathrm{E}+00$ & -0.360 & $1.0100 \mathrm{E}+00$ & -0.356 & 0.97 \\
\hline & 149686 & $8.2237 \mathrm{E}-01$ & -0.316 & $8.0092 \mathrm{E}-01$ & -0.310 & 0.97 \\
\hline
\end{tabular}

Table 13: Numerical errors, convergence order, and effectivity indices of the error estimator in $H$-curl norm (Adaptive refinement). 


\begin{tabular}{|c|c|c|c|c|c|c|}
\hline$\beta$ & $\operatorname{Dof}(V)$ & $\|\mathbf{u}-\hat{\mathbf{u}}\|$ & order & $\|\varepsilon\|$ & order & $\mathrm{EFF}$ \\
\hline \multirow{9}{*}{-1} & 52 & $8.4612 \mathrm{E}+00$ & & $5.7291 \mathrm{E}+00$ & & 0.68 \\
\hline & 334 & $8.6984 \mathrm{E}+00$ & 0.015 & $5.7495 \mathrm{E}+00$ & 0.002 & 0.66 \\
\hline & 2164 & $9.7104 \mathrm{E}+00$ & 0.059 & $8.9312 \mathrm{E}+00$ & 0.236 & 0.92 \\
\hline & 5218 & $3.5941 \mathrm{E}+00$ & -1.129 & $3.3856 \mathrm{E}+00$ & -1.102 & 0.94 \\
\hline & 8272 & $2.6783 \mathrm{E}+00$ & -0.638 & $2.5916 \mathrm{E}+00$ & -0.580 & 0.97 \\
\hline & 11326 & $1.9417 \mathrm{E}+00$ & -1.023 & $1.8669 \mathrm{E}+00$ & -1.044 & 0.96 \\
\hline & 21856 & $1.5911 \mathrm{E}+00$ & -0.303 & $1.5379 \mathrm{E}+00$ & -0.295 & 0.97 \\
\hline & 70138 & $1.0471 \mathrm{E}+00$ & -0.359 & $1.0163 \mathrm{E}+00$ & -0.355 & 0.97 \\
\hline & 151174 & $8.1930 \mathrm{E}-01$ & -0.319 & $7.9837 \mathrm{E}-01$ & -0.314 & 0.97 \\
\hline \multirow{9}{*}{$-10^{-2}$} & 52 & $4.3956 \mathrm{E}+01$ & & $4.3017 \mathrm{E}+01$ & & 0.98 \\
\hline & 334 & $6.1211 \mathrm{E}+01$ & 0.178 & $4.6957 \mathrm{E}+01$ & 0.047 & 0.77 \\
\hline & 2164 & $8.5672 \mathrm{E}+01$ & 0.180 & $8.4417 \mathrm{E}+01$ & 0.314 & 0.99 \\
\hline & 5218 & $2.3817 \mathrm{E}+01$ & -1.454 & $2.3987 \mathrm{E}+01$ & -1.430 & 1.01 \\
\hline & 7936 & $1.6834 \mathrm{E}+01$ & -0.828 & $1.6600 \mathrm{E}+01$ & -0.878 & 0.99 \\
\hline & 11326 & $3.3279 \mathrm{E}+00$ & -4.557 & $3.2476 \mathrm{E}+00$ & -4.587 & 0.98 \\
\hline & 14380 & $2.3459 \mathrm{E}+00$ & -1.465 & $2.2642 \mathrm{E}+00$ & -1.511 & 0.97 \\
\hline & 52780 & $1.6182 \mathrm{E}+00$ & -0.286 & $1.5656 \mathrm{E}+00$ & -0.284 & 0.97 \\
\hline & 74656 & $1.3089 \mathrm{E}+00$ & -0.612 & $1.2755 \mathrm{E}+00$ & -0.591 & 0.97 \\
\hline \multirow{9}{*}{$10^{-2}$} & 52 & $4.3989 \mathrm{E}+01$ & & $4.3063 \mathrm{E}+01$ & & 0.98 \\
\hline & 334 & $6.1212 \mathrm{E}+01$ & 0.178 & $4.6973 \mathrm{E}+01$ & 0.047 & 0.77 \\
\hline & 2164 & $8.5673 \mathrm{E}+01$ & 0.180 & $8.4420 \mathrm{E}+01$ & 0.314 & 0.99 \\
\hline & 5218 & $2.3818 \mathrm{E}+01$ & -1.454 & $2.3988 \mathrm{E}+01$ & -1.430 & 1.01 \\
\hline & 7936 & $1.6833 \mathrm{E}+01$ & -0.828 & $1.6599 \mathrm{E}+01$ & -0.878 & 0.99 \\
\hline & 11326 & $3.3279 \mathrm{E}+00$ & -4.557 & $3.2475 \mathrm{E}+00$ & -4.587 & 0.98 \\
\hline & 14380 & $2.3459 \mathrm{E}+00$ & -1.465 & $2.2641 \mathrm{E}+00$ & -1.511 & 0.97 \\
\hline & 52780 & $1.6181 \mathrm{E}+00$ & -0.286 & $1.5655 \mathrm{E}+00$ & -0.284 & 0.97 \\
\hline & 74656 & $1.3088 \mathrm{E}+00$ & -0.612 & $1.2754 \mathrm{E}+00$ & -0.591 & 0.97 \\
\hline \multirow{9}{*}{1} & 52 & $9.0601 \mathrm{E}+00$ & & $5.9403 \mathrm{E}+00$ & & 0.66 \\
\hline & 334 & $9.0556 \mathrm{E}+00$ & -0.000 & $5.7831 \mathrm{E}+00$ & -0.014 & 0.64 \\
\hline & 2164 & $9.7985 \mathrm{E}+00$ & 0.042 & $8.9340 \mathrm{E}+00$ & 0.233 & 0.91 \\
\hline & 5218 & $3.6038 \mathrm{E}+00$ & -1.136 & $3.3878 \mathrm{E}+00$ & -1.102 & 0.94 \\
\hline & 8272 & $2.6757 \mathrm{E}+00$ & -0.646 & $2.5827 \mathrm{E}+00$ & -0.589 & 0.97 \\
\hline & 11326 & $1.9435 \mathrm{E}+00$ & -1.018 & $1.8580 \mathrm{E}+00$ & -1.048 & 0.96 \\
\hline & 21856 & $1.5916 \mathrm{E}+00$ & -0.304 & $1.5350 \mathrm{E}+00$ & -0.290 & 0.96 \\
\hline & 70810 & $1.0419 \mathrm{E}+00$ & -0.360 & $1.0100 \mathrm{E}+00$ & -0.356 & 0.97 \\
\hline & 149686 & $8.2237 \mathrm{E}-01$ & -0.316 & $8.0092 \mathrm{E}-01$ & -0.310 & 0.97 \\
\hline
\end{tabular}

Table 14: Numerical errors, convergence order, and effectivity indices of the error estimator in energy norm (Adaptive refinement). 


\begin{tabular}{c||l|l|l}
\hline$\beta$ & Dof $(V)$ & Cnd. No $(V)$ & Cnd. No $(W)$ \\
\hline \multirow{5}{*}{-1} & 52 & 27.571 & 30.882 \\
& 334 & 238.616 & 47.811 \\
& 2164 & 910.456 & 85.699 \\
& 5218 & 1924.991 & 125.457 \\
& 8272 & 2594.674 & 141.581 \\
& 11326 & 3294.176 & 148.465 \\
& 21856 & 1721.462 & 149.520 \\
& 70138 & 2815.013 & 169.105 \\
& 151174 & 7597.905 & 167.481 \\
\hline \multirow{5}{*}{$-10^{-2}$} & 52 & 2228.669 & 24.427 \\
& 334 & 20500.691 & 45.240 \\
& 2164 & 54068.221 & 83.971 \\
& 5218 & 60490.684 & 124.584 \\
& 7936 & 61772.616 & 139.517 \\
& 11326 & 65341.786 & 148.170 \\
& 14380 & 66025.163 & 151.052 \\
& 52780 & 70900.218 & 172.075 \\
& 74656 & 49729.189 & 172.641 \\
\hline
\end{tabular}

Table 15: Condition numbers of the stiffness matrices for $V$ and $W$ (Adaptive refinement). 